Assembly and Magneto-Electrical Characterization of Hybrid Organic-Inorganic Systems 


\section{Assembly and Magneto-Electrical Characterization of Hybrid Organic-Inorganic Systems}


The research described within this thesis was carried out in the NanoElectronics Group at the MESA+ Institute for Nanotechnology at the University of Twente, Enschede, the Netherlands. The NWO VIDI program, grant no. 07580 and European Research Council (ERC) Starting Grant no. 240433 financially supported this research.

\section{UNIVERSITY OF TWENTE.}

MESA+

INSTITUTE FOR NANOTECHNOLOGY

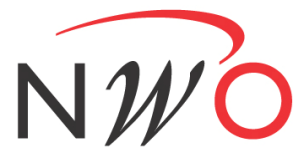

\section{Thesis committee members}

\section{Chairman\& secretary:}

Prof. dr. ir. A.J. Mouthaan

University of Twente

\section{Promotors:}

Prof. dr. ir. W.G. van der Wiel

University of Twente

Prof. dr. ing. D.H.A. Blank

University of Twente

\section{Other members:}

Prof. dr. S. Tarucha

University of Tokyo, Japan

Prof. dr. B. Koopmans

Eindhoven University of Technology

Prof. dr. ir. H.J.W. Zandvliet

University of Twente

Prof. dr. ir. J. Huskens

University of Twente

Prof. dr. ir. A. Brinkman

University of Twente

Title: Assembly and Magneto-Electrical Characterization of Hybrid OrganicInorganic Systems

Author: Tian Gang

Printed by Ipskamp Drukkers B.V., Enschede, The Netherlands, 2011.

Cover design and photography: Tian Gang 


\title{
ASSEMBLY AND MAGNETO-ELECTRICAL CHARACTERIZATION OF HYBRID ORGANIC-INORGANIC SYSTEMS
}

\author{
DISSERTATION
}

to obtain

the degree of doctor at the University of Twente, on the authority of the rector magnificus, prof. dr. H. Brinksma, on account of the decision of the graduation committee, to be publicly defended on Friday $28^{\text {th }}$ of October 2011 at 14:45

by

Tian Gang

born on December $19^{\text {th }}, 1983$

in Changchun, China 
This dissertation has been approved by:

Promotors:

Prof. dr. ir. W.G. van der Wiel

Prof. dr. ing. D.H.A. Blank

Copyright (C) 2011 by Tian Gang, Enschede, the Netherlands. All rights reserved.

ISBN: 978-90-365-3248-8

DOI: $10.3990 / 1.9789036532488$ 
This thesis is dedicated to my grandma, my parents, my wife and our lovely son. 



\section{Contents}

Chapter 1 Introduction

Chapter 2 Properties of magnetic systems as a function of size and 7 dimensionality

Chapter 3 Low-temperature solution synthesis of chemically functional ferromagnetic FePtAu nanoparticles

Chapter 4 Magnetic nanoparticle assembly on surfaces using clickchemistry

Chapter 5 Nano-patterned monolayer and multilayer of FePtAu nanoparticles on aluminum oxide substrate

Chapter 6 Transport properties of single FePt nanoparticles

Chapter 7 Towards hybrid organic-inorganic electron interferometers

Chapter 8 Tunable Molecular Spin Doping of a Metal

Appendix

Summary

Samenvatting

Acknowledgements

Author biography 



\section{Chapter 1}

\section{Introduction}

\subsection{Organic-inorganic hybrid electronics}

Inorganic matter can be defined with reference to organic matter. Inorganic literally means not-organic. This class of materials generally requires complex and powerful instruments to process. Crystalline inorganic materials are mostly used in electronic devices due to their superior electric and magnetic properties.

Organic materials are normally defined as those chemical compounds that contain carboni. These materials are extensively used in various technological applications that require easy processing, high flexibility and low cost. These characteristics motivated investigations to apply organic materials in electronic devices. This development started in 1963. High conductivity was found in iodine-doped and oxidized polypyrrole [1]. Since then, various potential organic electronic components were studied, including single molecules [2], self-assembled monolayers, organic thin films [3-6], organic single-crystals [7-9] and pure carbon materials [10-14]. Although the term "organic electronics" is often used, the actual devices normally consist of both organic and inorganic

\footnotetext{
i However several types of carbon-containing compounds such as carbides, carbon oxides, carbonates, cyanides and allotropes of carbon are classified as inorganic. The distinction between "organic" and "inorganic" carbon compounds is somewhat arbitrary.
} 
materials thus should be classified as organic-inorganic hybrid systems. These research efforts have led to ultra-light, flexible and cost-effective electronic applications [5-6]. Nowadays, electronic devices with organic electrical components have already been launched into the market (Fig. 1.1).

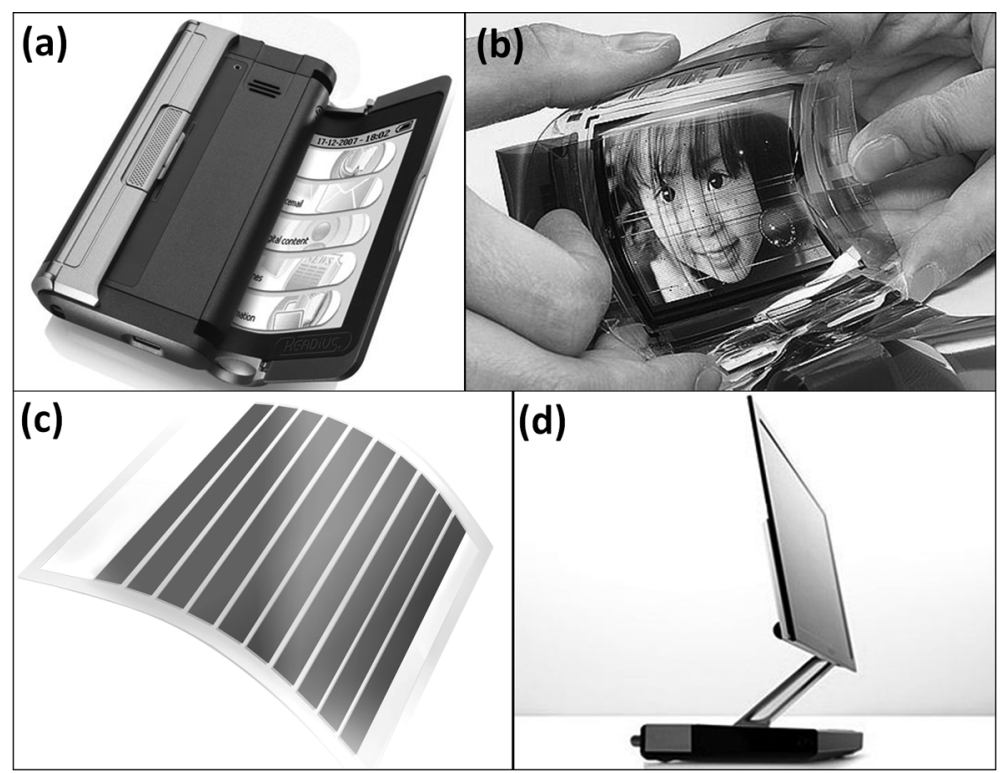

Figure 1.1: Applications of organic electronics. (a) Pocket eReader with roll able display (Polymer Vision). (b) Flexible organic light-emitting diode display (Sony). (c) Organic-dye solar cells (Heliatek). (d) First OLED television (Sony).

The possibilities of organic materials and surface chemistry in electronic devices have been investigated in many organic-inorganic hybrid systems [2-14]. The organic molecules are engineered at the atomic scale which results in chemical tuning of electronic functionality [15]. Various self-assembly and soft-lithography method have been developed based on surface chemistry [16]. Potentially, the bottom-up fabrication of electronic device can be realized in future. Furthermore, single molecule may eventually form the ultimately miniaturized electronic devices [17].

Several important issues still remain in the organic-inorganic hybrid systems. Imperfection and insufficient purity in organic material still limit its carrier mobility epically in polymer thin film base devices [18]. More control is required 
on the nature of organic-inorganic interface which is of crucial importance to transport properties [19]. A good physical contact does not necessarily imply good electrical contact. Smarter assembly techniques with well controlled organic-inorganic interface still need to be further developed for organic-inorganic hybrid electronics.

\subsection{Outline of this thesis}

In this thesis, assembly and magneto-electrical characterization of several hybrid organic-inorganic magnetically active systems are described. Chemically synthesized magnetic nanoparticles with organic ligands are applied as building blocks of electronic devices. The controlled assembly of these ferromagnetic nanoparticles could be of interest for low-cost and ultra-high density data storage applications. Self-assembled monolayers are introduced into electron interference based quantum transport studies. Self-assembled monolayers are great model systems, which has great tunability and precise control in molecular function, inter-molecular interaction, density and coverage.

Chapter 2 provides a concise theoretical background for the magnetic and electrical phenomena in magnetically active organic-inorganic hybrid systems described in this thesis. Nanomagnetism, spin-dependent transport, Coulomb blockade effect, Kondo effect and electron interference effect are discussed.

Solution synthesis of ferromagnetic FePtAu nanoparticles, capped with organic ligands, is discussed in Chapter 3. These nanoparticles have great potential in ultra-high density data storage applications. A systematic study of the preparation of the ferromagnetic nanoparticles in solution at relative low temperatures is carried out. This low-temperature solution annealing method preserves the organic ligands around the nanoparticles. This enables the further chemical assisted assembly and patterning of these magnetic nanoparticles.

Chapter 4 demonstrates the assembly of a monolayer of magnetic nanoparticles on a surface using "click chemistry". This is one step forward towards using magnetic nanoparticle in range of spintronic and data storage applications. Functionalized ligands and self-assemble monolayers (SAMs) modified substrates 
are used to link the magnetic nanoparticles on a silicon oxide substrate. The magnetic nanoparticles are assembled based on irreversible covalent interaction. Nanoparticle patterns are generated on surfaces via micro-contact printing ( $\mu C P)$. The magnetic properties of the nanoparticle assembly and patterns are studied as well.

Chapter 5 discusses nanoimprint lithography and nano-molding in capillaries to fabricate monolayers and multilayers of magnetic nanoparticle patterns. Patterns at the micrometer and nanometer scale are created on aluminum oxide substrates. Tunnel barriers consist of several nanometer of aluminum oxide or other metal oxide is widely used in numerous spintronic devices. A well-controlled patterning and assembly method to position the magnetic nanoparticles on the aluminum oxide or other metal oxide substrate is essential to realize nanoparticle based spintronic devices [20-23].

Transport studies on single magnetic nanoparticles assembled on chemical modified metallic and oxide substrates are shown in Chapter 6. This investigation can give insights in designing nanoparticles based spintronic devices. Scanning tunneling microscopy at high vacuum and low temperature is used to characterize a single magnetic nanoparticle electrically. Coulomb blockade was obtained in this organic-inorganic hybrid electronic system.

In Chapter 7, an electron interferometer investigation for coherent electron transport in organic molecules is discussed. An e-beam lithography defined sub-micrometer Aharonov-Bohm ring has chosen as a probe for coherent electron transport. A modified Aharonov-Bohm ring with a nano sized gap and a nano sized junction have been designed and fabricated. This may enable insertion of organic molecules into the electron interferometer via nanoparticle bridging. The efforts to realize such low level (nano-volt) cryogenic (250 mK) measurements are also described in this chapter.

Finally in Chapter 8, a two-dimensional spin system was fabricated combining both top down (metal sputtering) and bottom up (molecular self assembly) approaches. The system consists of disordered gold films with a monolayer of paramagnetic molecules. The self assembled monolayer introduces a robust way to define the concentration of magnetic impurities in this two-dimensional spin 
system. Using this unique platform, we studied the Kondo effect and coherent transport. Our efforts can provide insights in the electronic properties of a wide variety of materials where the interactions between electrons are particularly strong.

\section{References}

[1] B. A. Bolto, R. McNeill, D. E. Weiss, Australian Journal of Chemistry 16 (1963) 1090.

[2] M. A. Reed, C. Zhou, C. J. Muller, T. P. Burgin, J. M. Tour, Science 278 (1997) 252.

[3] H. Sirringhaus, P. J. Brown, R. H. Friend, M. M. Nielsen, K. Bechgaard, B. M. W. Langeveld-Voss, A. J. H. Spiering, R. A. J. Janssen, E. W. Meijer, P. Herwig, D. M. de Leeuw, Nature 401 (1999) 685.

[4] P. Peumans, S. Uchida, S. R. Forrest, Nature 425 (2003) 158.

[5] D. Voss, Nature 407 (2000) 442.

[6] S. R. Forrest, Nature 428 (2004) 911.

[7] V. C. Sundar, J. Zaumseil, V. Podzorov, E. Menard, R. L. Willett, T. Someya, M. E. Gershenson, J. A. Rogers, Science 303 (2004) 1644.

[8] V. Podzorov, E. Menard, A. Borissov, V. Kiryukhin, J. A. Rogers, M. E. Gershenson, Physical Review Letters 93 (2004)

[9] R. W. I. de Boer, M. E. Gershenson, A. F. Morpurgo, V. Podzorov, Physica Status Solidi a-Applied Research 201 (2004) 1302.

[10] T. Rueckes, K. Kim, E. Joselevich, G. Y. Tseng, C. L. Cheung, C. M. Lieber, Science 289 (2000) 94.

[11] C. Joachim, J. K. Gimzewski, A. Aviram, Nature 408 (2000) 541.

[12] R. H. Baughman, A. A. Zakhidov, W. A. de Heer, Science 297 (2002) 787.

[13] A. K. Geim, Science 324 (2009) 1530. 
[14] K. S. Novoselov, A. K. Geim, S. V. Morozov, D. Jiang, Y. Zhang, S. V. Dubonos, I. V. Grigorieva, A. A. Firsov, Science 306 (2004) 666.

[15] W. J. M. Naber, S. Faez, W. G. van der Wiel, Journal of Physics D-Applied Physics 40 (2007) R205.

[16] A. A. Tseng, A. Notargiacomo, Journal of Nanoscience and Nanotechnology 5 (2005) 683.

[17] G. Cuniberti, G. Fagas, K. E. Richter, Introducing Molecular Electronics Springer (2005)

[18] G. Brocks, J. van den Brink, A. F. Morpurgo, Physical Review Letters 93 (2004)

[19] K. W. Hipps, Science 294 (2001) 536.

[20] M. Acet, C. Mayer, O. Muth, A. Terheiden, G. Dyker, Journal of Crystal Growth 285 (2005) 365.

[21] S. H. Sun, S. Anders, H. F. Hamann, J. U. Thiele, J. E. E. Baglin, T. Thomson, E. E. Fullerton, C. B. Murray, B. D. Terris, Journal of the American Chemical Society 124 (2002) 2884.

[22] S. H. Sun, S. Anders, T. Thomson, J. E. E. Baglin, M. F. Toney, H. F. Hamann, C. B. Murray, B. D. Terris, Journal of Physical Chemistry B 107 (2003) 5419.

[23]A. C. C. Yu, M. Mizuno, Y. Sasaki, M. Inoue, H. Kondo, I. Ohta, D. Djayaprawira, M. Takahashi, Applied Physics Letters 82 (2003) 4352. 


\section{Properties of magnetic systems as a function of size and dimensionality}

\subsection{Introduction}

Nanoelectronics can be defined as applying nanotechnology to electronics components. In the industry, nanoelectronics explicitly refer to using nanotechnology to further reduce the size of the transistors to meet the rapid expanding information processing demands. Nanoelectronics is also considered as a disruptive technology, since its building blocks are significantly different from material in conventional semiconductor industry. Some of these candidates include: single molecules, molecular monolayers, organic single crystals or one dimensional nanotubes and nanowires [1].

The demands in information storage capacity keep boosting over the years along with the demands for increasing information processing power. Currently, electromagnetic devices play major roles in information storage applications. Such electromagnetic devices include hard drive, tape drive and MRAM (magnetoresistive random access memory) etc.

The growing demands in information storage capacity strongly motivated the research efforts on the properties of magnetic systems as a function of size and dimensionality. For bulk material, the magnetic behavior (hard or soft) depends 
on its microstructure. It is a matter of processing method and our understanding is mostly qualitative and empirical. However, as the dimensions approach the domain wall width (order nanometer), lateral confinement (shape and size) and single-electron charging effects are dominating. These parameters render the classical descriptions grossly inadequate. As the dimension further reduces to molecular level, intermolecular interactions and quantum mechanics dominates the properties of the system.

In this thesis, I describe the assembly and magneto-electrical characterization of several hybrid organic-inorganic magnetically active systems. One system is composed of FePt magnetic nanoparticles assemblies. These nanoparticles are a few nanometers in size. In this chapter, I will build up a theory background about the magnetism and single electron charging effect in the magnetic nanoparticles. The other system consists of molecules with unpaired spins. Several quantum phenomena dominating at this length scale will be discussed in this chapter, too.

\subsection{Nanomagnetism}

The key to understand nanomagnetism is the exchange correlation length. In case of magnetic nanoparticles, the magnetic properties vary dramatically as the size of nanoparticle is comparable to the magnetic domain size. This reduction in size and dimensionality leads to two types of magnetic properties in nanoparticles. One type is single domain ferromagnetic nanoparticles and the other type is superparamagnetic nanoparticles.

Both bulk ferromagnetic material and assemblies of single-domain magnetic nanoparticles show an increase in magnetization with an increase in external magnetic field. When all individual moments are aligned with external magnetic field, the net magnetization is defined as the saturation magnetization $\left(M_{\mathrm{s}}\right)$. After saturation, the specimen still has certain net magnetization when the external magnetic field is set to zero. This magnetization is defined as remanence $\left(M_{r}\right)$. To bring the remanent magnetization to zero, one needs to apply a reversed external magnetic field. The strength of the field required is named the coercive field $\left(H_{c}\right)$ (Fig. 2.1a). The mechanism how the net magnetization reacts to the 
external field is very different in bulk ferromagnetic materials and assemblies of single domain ferromagnetic nanoparticles.
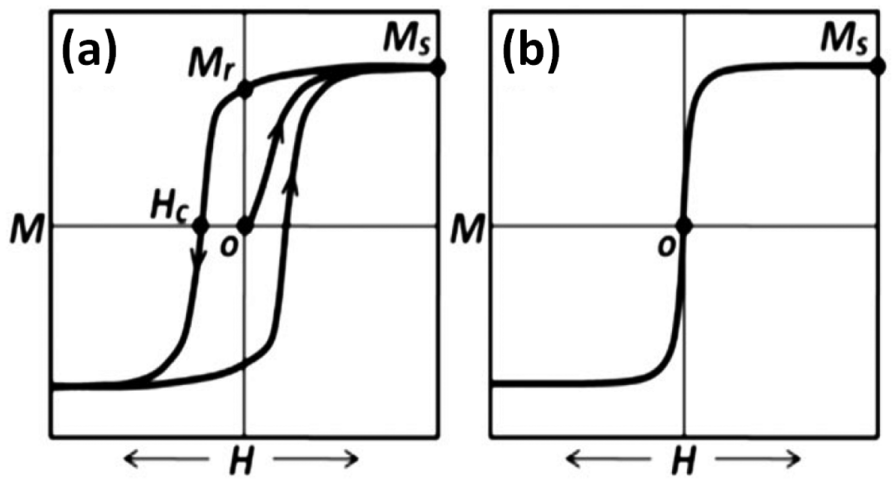

Figure 2.1: Schematic hysteresis loops for magnetic nanoparticle assemblies showing ferromagnetic behavior (a) and superparamagnetic behavior (b).

Without external magnetic field, bulk ferromagnetic material generally consists of multiple magnetic domains to minimize the overall (magnetostatic) energy. However, as the size of a magnetic nanoparticle gets small enough, the (exchange) energy necessary to form multiple magnetic domains is higher than the energy required to remain as a single magnetic domain. In bulk ferromagnetic materials, the net magnetization changes through domain growth as well as the rotation of the atomic magnetic moments. For a single-domain ferromagnetic nanoparticle, only coherent rotation of the atomic magnetic moments in the nanoparticle or the rotation of the nanoparticle can align the nanoparticle magnetization to the external magnetic field.

There is a critical size below which the energy favorable state of a magnetic particle is the single-domain state (Fig. 2.2a). As the particle diameter increases, the stray field energy $\left(\Phi_{s}\right)$ raises proportional to the volume of the particle. In Fig. $2.2 \mathrm{~b}$, a two-domain particle is formed to reduce $\Phi_{\mathrm{s}}$. Assuming the formation of a two-domain ellipsoidal particle reduces the $\Phi_{\mathrm{s}}$ by a factor $\alpha$, a comparison between the two states of this particle leads to

$D=\frac{3 \gamma_{\mathrm{B}}}{N(1-\alpha) M_{\mathrm{S}}^{2}}$ 
$\gamma_{\mathrm{B}}=4 \sqrt{A K}$,

where $N$ is the geometry factor, $M_{\mathrm{s}}$ is the saturation magnetization, $\gamma_{\mathrm{B}}$ is the specific wall energy, $A$ is the exchange constant and $K$ is the effective anisotropy constant [2]. For a spherical particle $N=4 \pi / 3, \alpha \approx 1 / 2$; adding these to Eq. 2.1 and 2.2 gives the critical diameter $(D)$

$D=\frac{9 \sqrt{A K}}{2 \pi M_{S}^{2}}$

For most magnetic particles, the critical diameter is in the range of $10-100 \mathrm{~nm}$. For ultra-high magnetic anisotropy materials the single-domain limit can extend to a few micrometers [2].

(a)

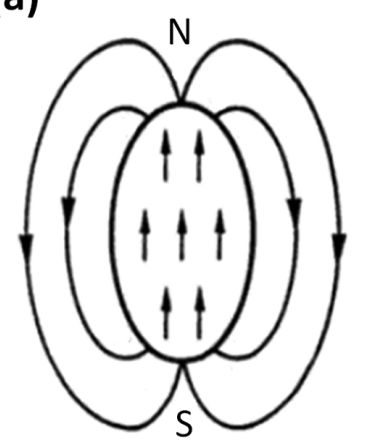

(b)

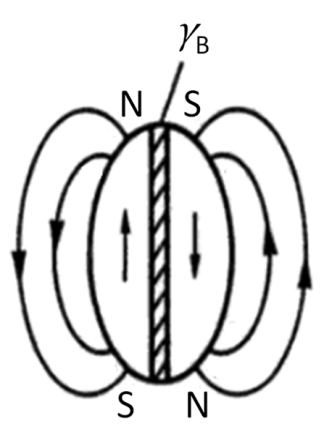

Figure 2.2: A single-domain magnetic nanoparticle (a) and a double-domain magnetic nanoparticle (b).

For a sufficiently small single-domain magnetic nanoparticle, the thermal energy can be large enough to rotate its magnetization vector over the energy barriers of $K V$ and $\Phi_{s}, V$ is the volume of the particle. In magnetic nanoparticle, the lifetime of a magnetic state is determined by the Arrhenius equation [3]

$\tau=\tau_{0} \exp \frac{K V}{k_{B} T}$,

here $\tau_{0}$ is the time interval of the particle magnetic moment attempt to jump between the opposite directions along the magnetization easy-axis. $\tau_{0}$ is characteristic of the material and in the order of $10^{-8}-10^{-10} \mathrm{~s}$. According to Eq. 2.4, thermal stability of the magnetic states is lost within very tight range of 
nanoparticle volumes. In such case, the magnetization vector of the nanoparticle is no longer stable. This situation is referred to the superparamagnetic state, because the magnetic behavior of the system in this state is analogous to classical paramagnetic materials. The individual moments of the superparamagnetic nanoparticles in the assembly can be saturated with an external magnetic field. The magnetization returns to zero as the removal of the external field due to thermal fluctuations. The temperature at which the thermal energy can overcome the anisotropy energy $K V$ of a magnetic nanoparticle is defined as the blocking temperature $\left(T_{\mathrm{B}}\right)$ [4]. Apart from the volume of the nanoparticle, $T_{\mathrm{B}}$ also depends on the monodispersity, exchange interactions and characteristic measurement time [5]. $T_{B}$ is the characteristic value that determines the transition point between ferromagnetic and superparamagnetic behavior for given magnetic nanoparticle assembly. Figures 2.1a and 2.1b give a schematic illustration of hysteresis curves for magnetic nanoparticle assemblies showing ferromagnetic and superparamagnetic behavior, respectively. According to Eq. 2.4, a thermally stable magnetic state can be achieved by either increasing the nanoparticle volume or by increasing the magnetic anisotropy constant.

\subsection{Spin-dependent transport}

One of the well studied phenomena of spin-dependent transport is the magneto resistance arise from the magnetic tunnel junction (MTJ). A MTJ generally consists of two layer of ferromagnetic material and a thin insulation layer as tunnel barrier in between (Fig. 2.3). In a MTJ, the density of states at the Fermi level on either side of the junction is spin-polarized and the tunneling current depends on the relative magnetic orientation of the two ferromagnetic layers (Fig. 2.3) [6-7]. Tunnel magneto resistance (TMR) is defined as the relative resistance change between parallel (P) and anti-parallel (AP) magnetic orientations

$T M R=\frac{R_{\mathrm{AP}}-R_{\mathrm{P}}}{R_{\mathrm{P}}}[6-7]$.

A typical TMR experiment consists of fully aligning the magnetization vector of the two ferromagnetic layers at high magnetic field and measuring the tunnel 
resistance in the parallel state. Then reversing the magnetic orientation of the bottom (or top) ferromagnetic layer and measuring the tunnel resistance again in the anti-parallel state. The two ferromagnetic layers should have well separated coercive fields to be able to switch separately.

(a)
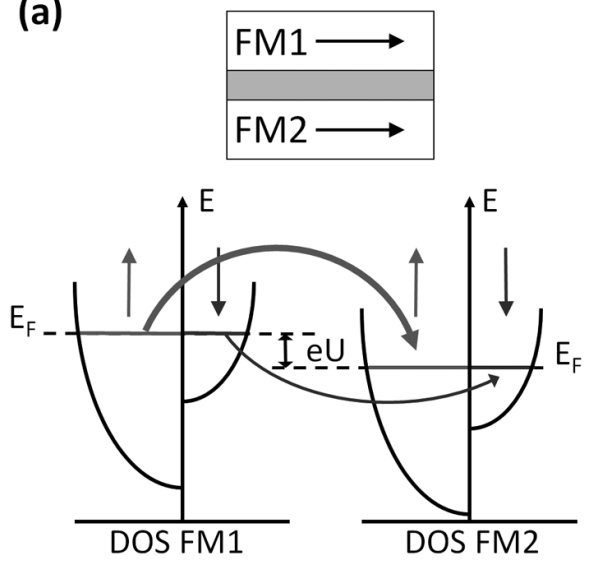

(b)
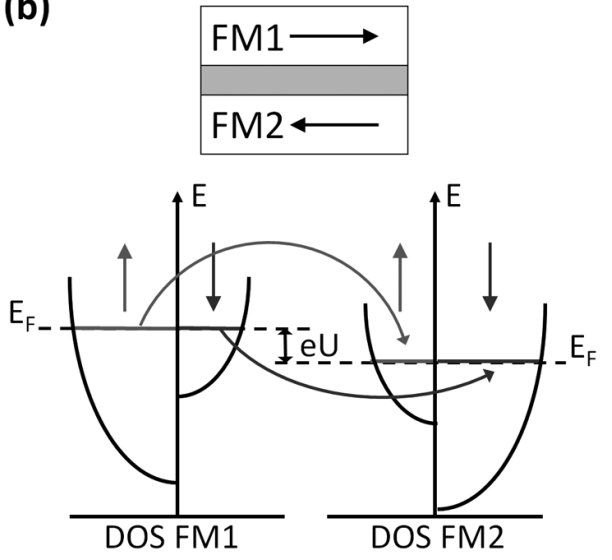

Figure 2.3: Magnetic tunnel junctions consisting of two ferromagnetic layers (FM) separated by an isolating barrier. A spin-polarized density of states (DOS), indicated by the arrows, leads to high tunneling conductance for the parallel orientation (a) and low tunneling conductance for the anti-parallel orientation (b) [6-7].

Figure 2.4 shows promising measurement geometry to study spin dependent tunneling through magnetic nanoparticle. It can be archived by combining chemically assisted nanoparticle assembly and scanning tunneling microscopy (STM) (see Chapter 6). This geometry is analogous to the vertical MTJ (Fig. 2.3). It consists of well-separated magnetic nanoparticles anchored on top of a ferromagnetic substrate capped with a tunnel barrier. A STM tip is applied as top contact, since the tip can be positioned exactly on top of a magnetic nanoparticle [8-10]. 
(a)

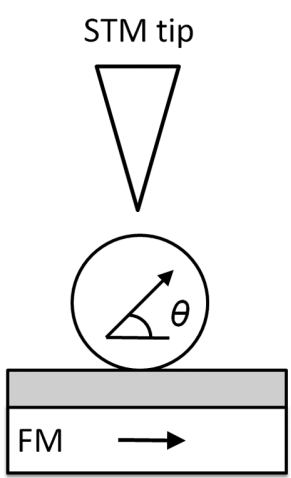

(b)

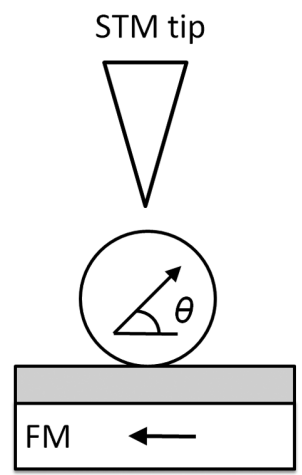

Figure 2.4: Scheme of the measurement geometry to study spin dependent transport through magnetic nanoparticle. The easy axis of the magnetic nanoparticle and the ferromagnetic substrate is misaligned with an angle $\theta$. The parallel state (a) and anti-parallel state (b) are presented.

The easy axis of the ferromagnetic substrate is in plan. It is due to the shape anisotropy of the thin film. However, the easy axis of the magnetic nanoparticle is random. This may lead to partial misalignment of the magnetization vector between the nanoparticle and the substrate layer (Fig. 2.4). The dependence of the tunneling conductance on the angle $\theta$ between the magnetization vectors of the two magnetic materials in an MTJ is given by

$G=G_{0}(1+\varepsilon \cos \theta)$,

where $G_{0}$ is a base conductance depending on geometric and material properties and the scaling factor $\varepsilon$ is a measure for the effective spin polarization in the device [11]. In a perfect system (100\% spin polarization) $\varepsilon$ would be unity. Using Eqs. 2.5 and 2.6, the TMR dependence on the easy axis alignment angle (assuming $\pi<\theta<\pi$ ) can be derived as

$\operatorname{TMR}(\theta)=\frac{1+\varepsilon \cos \theta}{1-\varepsilon \cos \theta}-1$.

According to Eq. 2.7, TMR is highest when the easy axis are completely aligned $(\theta=0)$, and decreases with increasing angle. In the extreme case where the nanoparticle easy axis is perpendicular to the substrate easy axis $\left(\theta=\frac{\pi}{2}\right)$ the TMR completely vanishes. 


\subsection{Coulomb blockade effect}

The proposed measurement geometry (Fig. 2.4) in Section 2.3 consists of two tunnel barriers. One barrier is between the STM tip and the nanoparticle. The other barrier is between the nanoparticle and the substrate. These two barriers define a double junction system. A generalized double junction system and corresponding circuit diagram is shown in Fig. 2.5a. Single electron tunneling (SET) can be observed in such double junction system through the effect know as Coulomb blockade [12].
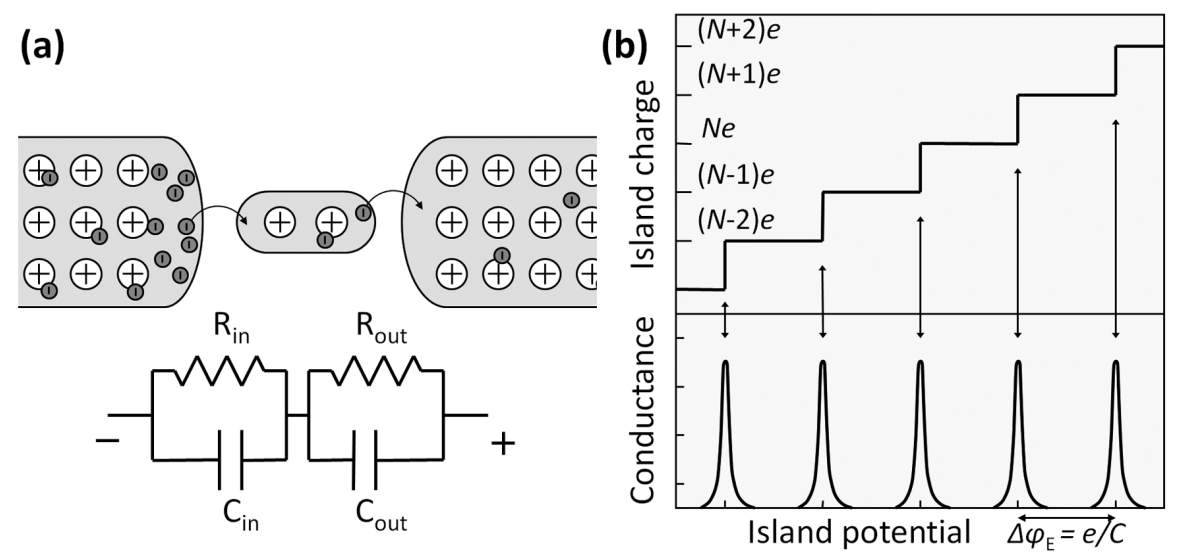

Figure 2.5: Schematic view of a generalized double tunnel junction enabling single-electron tunneling onto an isolated node. The equivalent electrical schematic consists of capacitive coupling and DC tunneling resistances (a). SET leads to an integer number of electrons on the island, depending on the node potential (b) [12].

The origin of Coulomb blockade of this double tunnel junction can be found at the node between two barriers (Fig. 2.5a). The electron can only get in and out of the isolated node through tunneling. The charge state of the node can only be an integer amount of the elementary charge: $Q=N \cdot e(N \in Z)$. For an isolated node capacitively coupled to its surroundings, the total energy stored in the nanoparticle can be expressed as the sum of the charging energy and the potential energy

$E(N)=\frac{(N \cdot e)^{2}}{2 C}-\varphi_{\mathrm{E}} \cdot N \cdot e$, 
where $C$ is the total capacitance between the node and its surroundings and $\varphi_{\mathrm{E}}$ is the electrostatic potential of the node [13]. The critical potential $\varphi_{\mathrm{E}_{\text {_crit }}}$ required for adding one more electron can be obtained by solving equation $E(N+1)=\mathrm{E}(N)$, yielding

$\varphi_{\text {E_crit }}=\frac{e \cdot(2 N+1)}{2 C}$,

since $N$ can only be integer, the number of electrons on the isolated node remains constant if $\varphi_{\mathrm{E}}$ is kept between two critical values

$\varphi_{\mathrm{E}_{\text {crit }}}(N) \leq \varphi_{\mathrm{E}}<\varphi_{\mathrm{E}_{\text {crit }}}(N+1)$.

This leads to the step-like relationship between the tunneling current and bias voltage as shown in Fig. 2.5b. This characteristic relationship is also known as the Coulomb staircase. The potential difference between two steps can be derived as

$\Delta \varphi_{\mathrm{E}}=\varphi_{\mathrm{E}}(N+1)-\varphi_{\mathrm{E}}(N)=\frac{e(2(N+1)+1)}{2 C}-\frac{e(2 N+1)}{2 C}=\frac{e}{C}$.

At non-zero temperature the total charge on the isolated node is not governed solely by the electrostatic potential. Thermal activation allows electrons to tunnel even if the potential on the node is lower than the critical value. Observing the Coulomb blockade therefore requires the thermal energy $\left(E_{t}\right)$ to be much lower than the Coulomb charging energy,

$E_{t}=k_{B} \cdot T \ll E_{c}=e \cdot \Delta \varphi_{\mathrm{E}}=\frac{e^{2}}{C}$,

where $k_{B}$ is the Boltzmann constant and $T$ the temperature in Kelvin. This shows that the capacitive coupling to the island must be sufficiently small; with thermal energy of several meV, the capacitance required to observe Coulomb blockade is in the order of $10^{-18}$ farads.

A second requirement to measure Coulomb blockade is that the electrons must be strongly confined to the island. This condition can be met if the tunnel barriers are sufficiently opaque, thus if the tunnel resistances are high. To estimate the minimum required tunnel resistance, we assume the characteristic time for charge fluctuations at the node is $\delta \tau \simeq R C$, where $C$ is the total capacitance between the isolated node and the surrounding, $R$ the tunnel resistance by which the electron is tunnel in or out the isolated node. Using the Heisenberg energy/time uncertainty relation, the energy uncertainty is given 
by $\delta E=\frac{h}{\delta \tau}=\frac{h}{R C}$. Furthermore, for a strong electron confinement, the electron energy uncertainty should be smaller than the Coulomb charging energy $\delta E<\frac{e^{2}}{C}$, which then reduces to $R>\frac{h}{e^{2}}=26 \mathrm{k} \Omega$.

So far, we assume the charge state of the nanoparticle is only governed by the tunneling of electrons into the isolated nanoparticle. However for observing Coulomb staircase (Fig. 2.5b), one should also consider the tunneling of electrons out of the isolated nanoparticle. The Coulomb staircase is observed most clearly if the tunneling rate into the nanoparticle is much higher than the rate out of the nanoparticle. This requirement can be understood if we consider the Coulomb charging of the isolated nanoparticle. If the outbound tunneling rate is very high, the mean occupation time decreases and the time-averaged charging drop, reducing the blockade effect.

As has become clear from the previous discussion, the junction capacitances play a vital role in Coulomb blockade experiments. In first order approximation small islands can be modeled as an isolated spherical conductor with self-capacitance

$C=\frac{Q}{\Delta V}=4 \pi \cdot \varepsilon_{0} \cdot \varepsilon_{r} \cdot R$,

where $\Delta V$ is the potential difference between the sphere (radius $R$ ) and a spherical conducting shell surrounding it, $\varepsilon_{r}$ is the relative permeability of the material between the sphere and a spherical conducting shell surrounding it, $\varepsilon_{0}$ is the permeability in vacuum. In a realistic device, the nanoparticle is never truly isolated from its surroundings, so to improve this model we can compute the capacitance between a sphere (e.g. a nanoparticle) and a plane (e.g. a substrate surface) according to Ref. [14]

$C=4 \pi \cdot \varepsilon_{0} \cdot \varepsilon_{r} \cdot R \cdot \sinh \alpha \sum_{n=2}^{\infty} \frac{1}{\sinh (n \cdot \alpha)}, \alpha=\cosh ^{-1}\left(1+\frac{d}{R}\right)$,

where $d$ is the particle-substrate gap size. 


\subsection{Kondo effect}

An anomalous resistivity minimum in metals at low temperature was observed when a dilute concentration ( $10 \mathrm{ppm}$ ) of magnetic impurities is present [15]. The theory that describes this anomaly was initiated by the work of Jun Kondo in 1964 [16], hence the Kondo effect. The Kondo effect arises from the interaction between a localized spin and many electrons in the surrounding Fermi Sea. As a result, this system is a many-body problem.

The resistivity of a pure metal normally drops with decreasing temperature due to the reduction of electron-phonon interactions. Normal metals (e.g. gold) maintain a finite resistivity even at lowest accessible cryogenic temperatures. This finite resistivity comes from the electron scattering events from static defects or impurities, which hinder the travel of electrons through the crystal.

A simple model of a magnetic impurity in a Fermi sea was introduced by Anderson in 1961 (Fig. 2.6) [17]. This model has only one energy level $\left(\varepsilon_{0}\right)$ below the Fermi energy. The level is occupied by a single spin- $1 / 2$ electron, which has a fixed spin, either spin-up or spin-down. The electron can escape from this trap with an additional energy $\varepsilon_{0}$, otherwise it is trapped. In classical mechanics, it is forbidden to take the electron out of the trap without paying excitation energy $\varepsilon_{0}$. In quantum mechanics, such configuration is allowed for a very short time. According to Heisenberg's uncertainty principle, this time scale is $\sim h /\left|\varepsilon_{0}\right|$. Within this time window, another electron in the Fermi sea must tunnel into the trap to satisfy energy conservation. However, the spin of this electron may have an opposite direction compared to the one escaped from the trap. Hence, the initial and final state of the magnetic impurity spin may be different. In reality, many of such exchange processes are possible. A Kondo resonance is created with the same energy as the Fermi level by those processes. The Kondo resonance causes highly efficient scattering of electrons with energies close to the Fermi level. These electrons are also dominating the conductivity at low temperature, and the strong scattering consequently leads to a significant contribution to the resistivity at low temperature. 


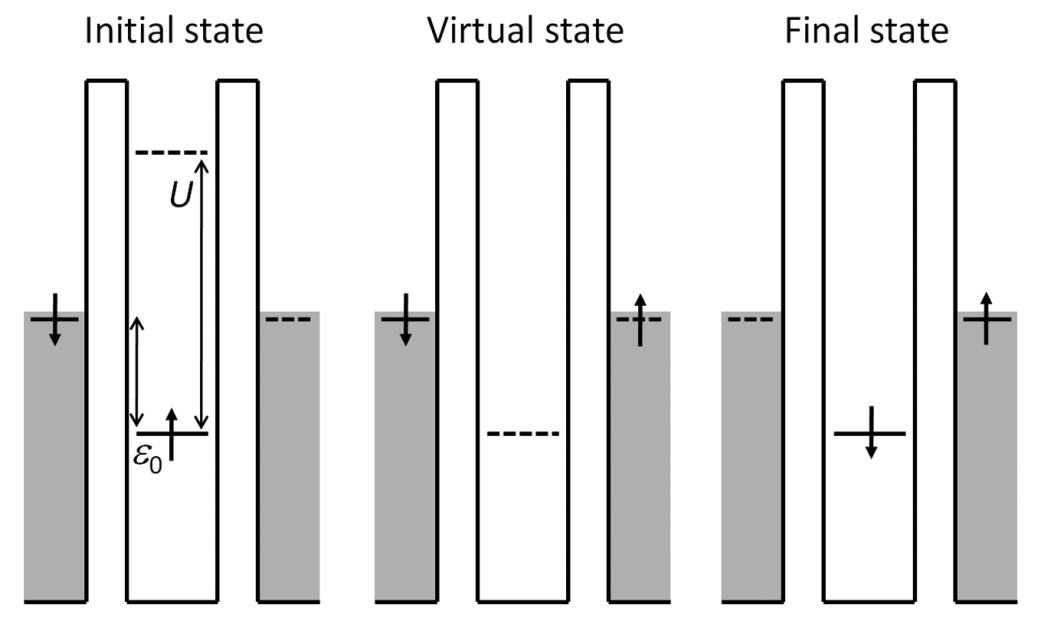

Figure 2.6: Schematic of the Anderson impurity model describing a single spin- $1 / 2$ impurity coupled to the Fermi sea [17].

The Kondo temperature $T_{\mathrm{K}}$ gives the characteristic energy of the Kondo effect. In the other word, the Kondo contribution to resistivity is domination at energy below $T_{\mathrm{k}}$. The Kondo temperature is related to the parameter of the Anderson model by

$T_{\mathrm{K}}=\frac{1}{2} \sqrt{\Gamma U} \exp \left[\frac{\pi \varepsilon_{0}\left(\varepsilon_{0}+U\right)}{\Gamma U}\right]$,

where $\Gamma$ is the width of the energy level and $U$ the Coulomb repulsion energy between two electrons at the impurity site.

Around a magnetic impurity, many electrons participate in the spin flip processes. Since all these electrons interact with the same magnetic impurity, they are correlated with each other. The collection of these correlated electrons is the so-called Kondo cloud. The spatial extension of the Kondo cloud is characterized by the Kondo length $\left(\mathcal{E}_{K}\right)[18]$. An estimate of the Kondo length is

$\varepsilon_{K}=\frac{\hbar v_{\mathrm{F}}}{k_{\mathrm{B}} T_{\mathrm{K}}}$,

where $v_{\mathrm{F}}$ is the Fermi velocity, and $\hbar$ the reduced Planck constant. The actual Kondo length, however, is still under debate. The experimental determination of the Kondo cloud is very challenging and considered as the "Holy Grail" in the 
field of Kondo physics [19]. When the concentration of magnetic impurities increases, the Kondo clouds around the impurities start to approach each other. Eventually, the magnetic impurities can couple to each other through conduction electrons, a phenomenon referred to Ruderman-Kittel-Kasuya-Yosida (RKKY) interaction [20-22]. In this configuration, the spin flip process is obstructed and the Kondo effect is suppressed.

By varying the magnetic impurity density, and thus their average separation, in a given metal, one can study the competition and transition between the Kondo and RKKY regime. This potentially can give a clue of the Kondo length. The precise control of the density of isolated magnetic impurity is the key issue in such experiment.

\subsection{Aharonov-Bohm effect and universal conductance fluctuations}

In this thesis I describe an investigation on the Aharonov-Bohm electron interferometer for the purpose of studying coherent electron transport in organic molecules. By inserting organic molecules into an electron interferometer, the coherency of electron transport through the organic molecule can be probed. In this section, a theoretical background of Aharonov-Bohm electron interferometer is presented.

In classical mechanics, the motion of an electron is not affected by the presence of a magnetic field in regions from which the electron is excluded. However, this is not the case in quantum mechanics. The Schrödinger equation for a charged particle $q$ in a magnetic field is

$\left\{\frac{1}{2 m}[\hat{p}-q A(R, t)]^{2}+q \phi(R, t)\right\} \psi(R, t)=i \hbar \frac{d}{d t} \psi(R, t)$,

here $m$ is the electron mass, $A$ the vector potential and $\hat{p}$ the canonical momentum. The canonical momentum $\hat{p}$ is represented by the operator $-i \hbar \frac{\partial}{\partial x}$, which appears as a spatial derivative. Phase of electron waves is defined as the fraction of a wave cycle which has elapsed relative to an arbitrary point, thus the spatial derivative canonical momentum $\hat{p}$ defines the phase information of the electron. Therefore, the electron phase can be affected by a 
magnetic field through the vector potential, even when the electron is spatially excluded from the magnetic field.

An ideal device to measure this phenomenon is shown in Fig. 2.7a. It is a ring shape electron conductor coupled to two electron reservoirs. An out of plane magnetic field presents only in the center of the ring. The magnetic field is zero at the wave guild area; however there is still a vector potential " $A$ " along the two electron paths. The electron is not passing through the magnetic field, so its mechanical momentum $p$ is constant. The mechanical momentum is a sum of the canonical momentum and the contribution from the vector potential:

$p=\hat{p} \pm e A$.

The partial electron waves travelling through the two different paths obtain two different phases due to the local vector potentials having opposite sign. The relative phase difference $(\Delta \phi)$ is given by

$\Delta \phi=\phi_{1}-\phi_{2}=2 \pi\left(\frac{e}{h}\right) \oint \vec{A} \cdot d \vec{l}=2 \pi\left(\frac{e}{h}\right) \oiint(\vec{\nabla} \times \vec{A}) \cdot d \vec{S}$,

with $\vec{\nabla} \times \vec{A}=\vec{B}$ and $\oiint(\vec{B} \cdot d \vec{S})=\phi$.

hence, the relative phase difference becomes,

$\Delta \phi=2 \pi\left(\frac{e}{h}\right) B S_{\text {in }}=2 \pi\left(\frac{\phi}{\phi_{0}}\right)$,

Here, $B$ is magnetic field, $S_{\text {in }}$ the enclosed area of the ring, $\phi$ the magnetic flux through the enclosed area, $\phi_{0}=h / e$ is the flux quantum, $\phi_{1}$ is the phase of the partial electron wave traversing through the upper arm, $\phi_{2}$ is the phase of the partial electron wave traversing through the lower arm. 


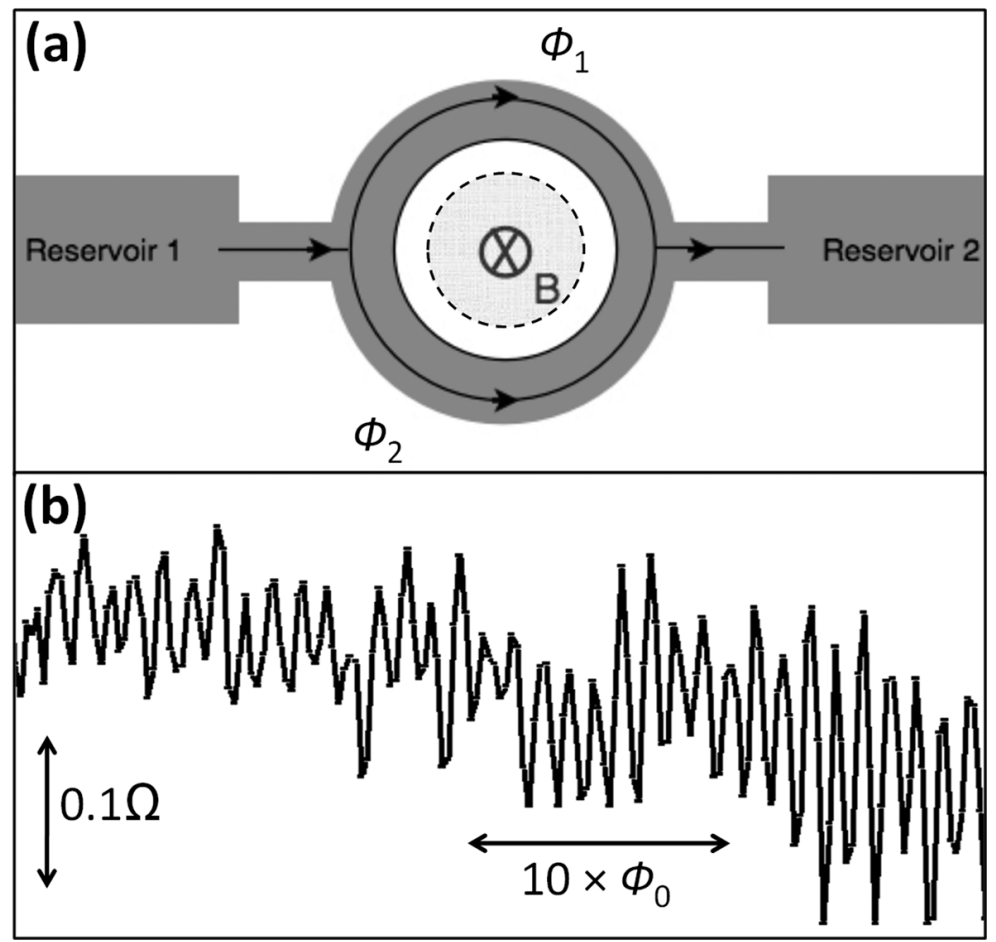

Figure 2.7: Schematic Aharonov-Bohm ring (a), an out of plane magnetic field is only present within the dashed circle at the center of the ring; magnetoresistance of a metallic ring measured at $T=262 \mathrm{mK}$ (b). The detail of this measurement is described in Chapter 7 .

The amplitude of the resulting electron wave function (when the two partial electron waves meet again and interfere) depends on both the amplitude and the phase of those two partial electron waves. According to Eq. 2.21, the relative phase difference varies between 0 and $2 \pi$ as function of magnetic field. This magnetic-field-dependent relative phase difference will affect the interference at the right side of the ring (Fig. 2.7a), thus the transmission probability, and hence the resistance of the ring. Sweeping the magnetic field continuously, one can measure resistance oscillations (Fig. 2.7b). The oscillation period is

$\Delta B=\frac{\phi_{0}}{S}=\frac{h}{e S}$.

The oscillation magnitude is in the order of $e^{2} / h$, independent of the total number of transport channels. As a result, the $A B$ effect is more pronounced 
when the total amount of conduction channels is low. This phenomenon was first predicted by Yakir Aharonov and David Joseph Bohm [23], hence the Aharonov-Bohm effect. The first experimental verification was on a single diffusive metal ring by Webb et al. in 1985 [24].

In a practical measurement, the magnetic field cannot be restricted to the center of the ring. The entire device is in the magnetic field instead. This imperfection leads to another quantum interference phenomenon named universal conductance fluctuations (UCF). Due to inhomogeneous scattering sites in the diffusive conductor, electrons can interfere. UCF have the same origin as the $A B$ effect; however UCF occur in a poorly defined geometry. As the geometry of the interference paths is not well defined in the case of UCF, one gets fluctuations instead of oscillations. According to the UCF theory [25], the conductance of any (semi-)conducting sample will be fluctuating as a function of chemical potential or magnetic field on the order of $e^{2} / h$, independent of sample size and degree of disorder as long as the coherency is maintained.

\subsection{Weak (anti) localization and electron-electron interaction}

Both the Kondo effect and the Aharonov-Bohm effect give a quantum correction to the conductivity at low temperatures. There are two other quantum phenomena named weak (anti) localization and electron-electron interaction that alter the conductivity.

As mentioned in Section 2.5, crystal imperfections in a conductor lead to electron scattering events which lead to increased resistance. Elastic and inelastic scattering are two fundamentally different phenomena. After an elastic scattering event, the electron preserves its energy, thus the phase of the electron is unchanged. In contrast, after inelastic scattering events, the energy of the electron changes and the phase of the electron is randomized. The inelastic scattering events include collisions of electron with phonons and other electrons.

The quantum correction to the conductivity needs to be considered when $\tau_{\varphi}>\tau$, where $\tau_{\varphi}$ is the coherence time and $\tau$ is the elastic scattering time.

The origin of the conductivity correction is the quantum coherent nature of the 
electrons, which also leads to the AB effect and UCF discussed in Section 2.6. Let us assume an electron diffuses in a coordinated system (Fig. 2.8a). The process starts at $t=0$ and at the origin $r=0$, with the Fermi velocity $v_{\mathrm{F}}$. After time $t \gg \tau$, the classical probability (Fig. 2.8b) to find this electron at position $r$ is given by

$P(r, t)=(4 \pi D t)^{-d / 2} e^{-r^{2} / 4 D t}$,

where $d$ is the dimensionality of the system and $D$ is the diffusion coefficient $\left(D=\frac{v_{\mathrm{F}}^{2} \tau}{d}\right)[26]$.

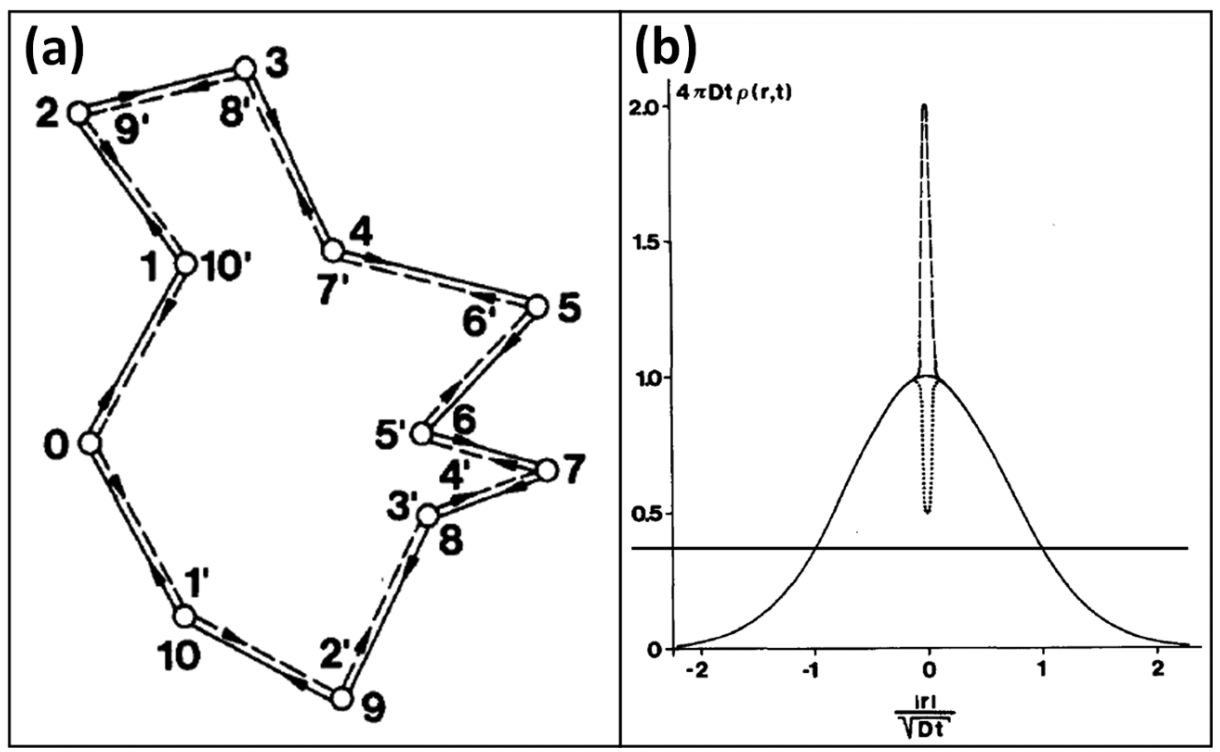

Figure 2.8: (a) A pair of loop like electron diffusion paths; both paths have an equal amount of scattering events. The propagation direction is opposite [26]. The probability amplitudes of these two time-reversed trajectories $\left(A_{1}\right.$ and $\left.A_{2}\right)$ are equal. (b) Probability distribution of a diffusing electron, starting from the origin $r=0$ at $t=0$. The solid plot is the classical prediction, the dashed peak is the correction from the weak localization and the dotted peak is the correction from the weak antilocalozation (see text).

Now we focus on time-reversed electron trajectory pairs, starting at $r=0$, and returning to $r=0$. These time-reversed trajectories have a loop like path and an equal amount of scatter events. When looking at these time-reversed trajectories, the classical probability of returning to the origin $r=0$ is a sum of the probabilities amplitudes from all possible trajectories $\left|A_{1}\right|^{2}+\left|A_{2}\right|^{2}=2 A^{2}$. However, when the electron wave behavior is considered, the wave functions 
(instead of the amplitudes) should be summed up for $t<\tau_{\varphi}$. Now, the probability of returning to the origin $r=0$ is $\left|A_{1}+A_{2}\right|^{2}=4 A^{2}$. Therefore, the probability that an electron is backscattered to $r=0$ is doubled compared to the classical prediction (Fig. 2.8b). The higher backscattering probability means a reduced transmission coefficient and gives a conductivity correction called weak localization. The relative magnitude of this contribution in a 2D system is given by

$\frac{\delta \sigma_{2}}{\sigma} \simeq-\int_{\tau}^{\tau_{\varphi}} \frac{v_{\mathrm{F}} \lambda^{2} d t}{(D t) b} \simeq \frac{v_{\mathrm{F}} \lambda^{2}}{D b} \ln \left(\frac{\tau_{\varphi}}{\tau}\right) \simeq \frac{1}{\left(k_{\mathrm{F}} l\right)\left(k_{\mathrm{F}} b\right)} \ln \left(\frac{\tau_{\varphi}}{\tau}\right), b \ll l_{\varphi}$,

where $b$ is the thickness of the metal film, $\lambda$ is the de Broglie wavelength and $k_{\mathrm{F}}$ is the Fermi wave vector. The conductivity contribution in Eq. 2.24 has a negative sign. Therefore, starting at a certain temperature (satisfying $l_{\varphi} \gg l$ ), the conductivity of the sample decreases as the sample is cooled down. The weak localization effect is observed widely in disordered metallic thin films such as $\mathrm{Cu}$ thin films (Fig. 2.9a) [27].

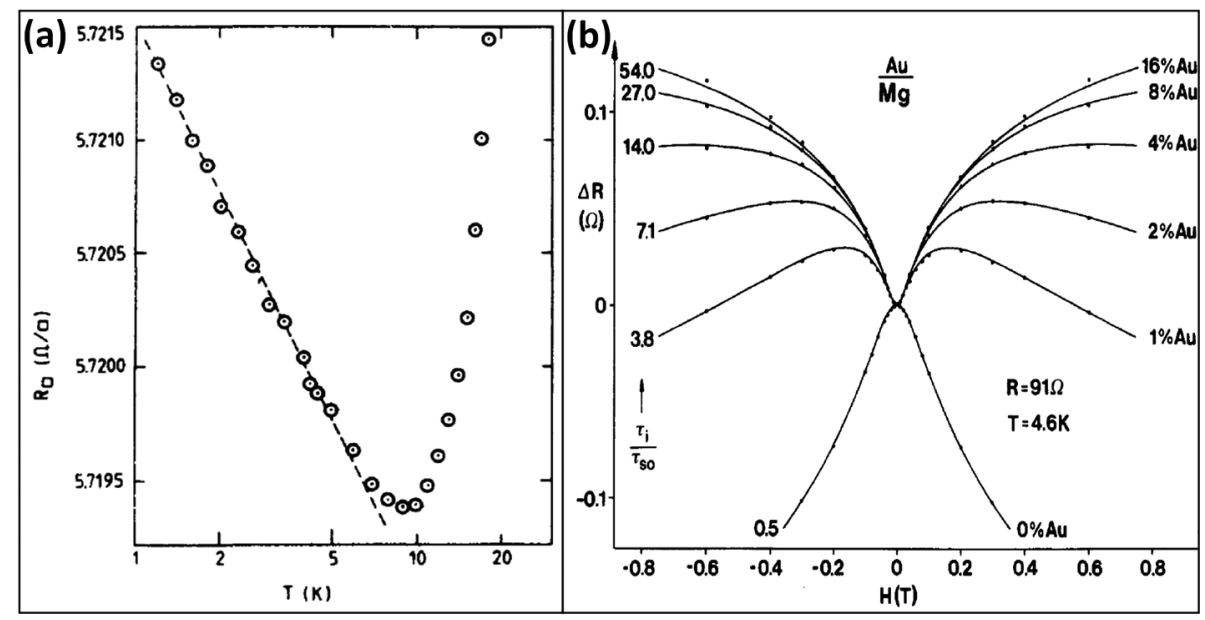

Figure 2.9: Temperature dependence of $\mathrm{Cu}$ film resistivity (a) [27]; the magneto-resistance of an $\mathrm{Mg}$ thin film measured at 4.5K for different coverage of $\mathrm{Au}$ on top (b) [28]. The ratio $\tau / \tau_{\text {so }}$ on the left side gives the strength of the spin-orbit scattering. It is essentially proportional to the Au-thickness.

As discussed above, the weak localization effect is caused by an electron interfering with itself. For such electron interference effect combine with a loop 
like electron path, it is natural to consider the effect from an external, perpendicular magnetic field. From Section 2.6, it is clear that, an electron moving along a loop like path in a perpendicular magnetic field gains an additional phase due to the vector potential $\vec{A}$ (Eq. 2.25).

$\psi \rightarrow \psi \exp \left(i \frac{e}{\hbar c} \int \vec{A} \cdot d \vec{l}\right)=\psi \exp \left( \pm \frac{i \pi B S}{\phi_{0}}\right)$,

here $S$ is the projected area of the loop to the plane perpendicular to the direction of the magnetic field. The phase shift of an electron propagating for time $t$ is given by

$\varphi=2 \pi\left(\frac{B S}{\phi_{0}}\right) \simeq \frac{B D t}{\phi_{0}}, t<\tau_{\varphi}$.

Therefore, under the external magnetic field, the probability for an electron returning to $r=0$ is rewritten as $\left|A_{1}+A_{2}\right|^{2}=\left|A_{1}\right|^{2}+\left|A_{2}\right|^{2}+2\left|A_{1}\right|\left|A_{2}\right| \cos \varphi=$ $2 A^{2}(1+\cos \varphi)$. In a finite magnetic field, the phase shift $\varphi$ becomes uncertain, since $S$ varies for different loop like electron trajectories. This leads to $\overline{\cos \varphi}=0$. In other words, the external magnetic field destroys the interference, thus increasing the conductivity (Fig. 2.9b). According to Eq. 2.26, the longer $t$, the smaller the critical magnetic field $\left(B_{\varphi}\right)$ needed to destroy coherent backscattering. The upper limit of $t$ is the coherence time $\tau_{\varphi}$, since beyond $\tau_{\varphi}$ the electron loses its phase memory. By assuming the interference starts to break down at $\varphi=1$, and using $t=\tau_{\varphi}$, the critical magnetic field of the system can be estimated from Eq. 2.26 and results in

$B_{\varphi} \simeq \frac{h}{e D \tau_{\varphi}}$.

For a thin homogeneous film with thickness $b$, the phase coherence length

$l_{\varphi}=\sqrt{D \tau_{\varphi}} \simeq \sqrt{\frac{h}{e B_{\varphi}}}, \quad b \ll l_{\varphi}$.

In the discussion above it is assumed that no spin flip occurs within the coherence time. However, in a heavy metal like gold, the spin-obit interaction may result in spin flip at elastic scattering acts. This leads to a positive contribution to the conductivity which is opposite to the conductivity contribution from weak localization. Therefore such phenomena, named as weak antilocalization. An electron with a fixed spin has a finite magnetic momentum $\mu$. 
When an electron with fixed spin moves with speed $v$, it generates an electric field $e \propto[\mu e]$ [28-29]. This field interacts with the ions charges in the heavy atom, which may result in spin flip at elastic scattering events. The spin-orbit interaction time $\tau_{\text {so }}$ depends on the electric field generated by the ions charges, and is thus insensitive to the temperature. As the temperature decreases, the system can enter the regime where $\tau \ll \tau_{\text {so }} \ll \tau_{\varphi}$. In this case, the calculation of the quantum conductivity correction needs to consider the sum of the wave functions of electrons with different spins [28-29]

$\frac{\delta \sigma_{2}}{\sigma} \simeq-\int_{\tau}^{\tau_{\varphi}} \frac{v_{\mathrm{F}} \lambda^{2} d t}{(D t) b}\left(\frac{3}{2} e^{-\frac{t}{\tau_{\mathrm{So}}}}-\frac{1}{2}\right)$

When $\tau \ll \tau_{\text {so }} \ll \tau_{\varphi}$, the term $\frac{3}{2} e^{-\frac{t}{\tau_{\text {so }}}}$ in Eq. 2.29 becomes negligible. Now only the second negative term is left, which results in a positive contribution to the conductivity. Experimental studies have proven the positive conductivity contribution from spin-orbit interaction by measuring the magnetoresistance (Fig. $2.9 b$ ) [28].

It is important to note that, the spin flip of an electron in elastic scattering evens with a nonmagnetic impurity leaves no trace in its surrounding quantum system. Thus, this electron wave still preserves its phase information. It still takes part in the interference and leads to weak antilocalization. In the case of elastic scattering events with a magnetic impurities accompanied by a electron spin flip, the impurity spin simultaneously flips due to total spin conservation in the system. This leaves a trace in the surrounding quantum system. In the other word, the electron exchanges information with the magnetic impurity during the scattering events. Consequently, scattering events with magnetic impurity cause de-coherence to the electron wave and result in negative contribution to $l_{\varphi}$.

Finally, I will briefly discuss the conductivity correction caused by electron-electron interaction. Weak (anti)localization arises from an electron interfering with itself. Interference can also happen between two different electrons. The details are not discussed in this thesis and can be found elsewhere [29]. Electron-electron interaction gives a very similar negative conductivity correction as weak localization. So it is very hard to distinguish the conductivity contribution from electron-electron interaction and weak localization. 
In Chapter 8, a 2D spin system is studied on a thin gold film $\left(d \ll l_{\varphi}\right)$ which shows weak-antilocalization phenomena at relative low magnetic field. Taking advantage of the sign difference of the conductivity correction; the contribution of weak-antilocalization can be well separated from the contribution of electron-electron interaction.

\section{References}

[1] W. J. M. Naber, S. Faez, W. G. van der Wiel, Journal of Physics D-Applied Physics 40 (2007) R205.

[2] H. Kronmüller, M. Fähnle, Micromagnetism and the Microstructure of Ferromagnetic Solids Cambridge University Press (2003)

[3] L. Néel, N. Kurti, Selected Works of Louis Neel Gordon and Breach (1988)

[4] L. Neel, Comptes Rendus Hebdomadaires Des Seances De L Academie Des Sciences 228 (1949) 664.

[5] S. A. Majetich, M. Sachan, Journal of Physics D-Applied Physics 39 (2006) R407.

[6] I. Zutic, J. Fabian, S. Das Sarma, Reviews of Modern Physics 76 (2004) 323.

[7] J. G. J. Zhu, C. D. Park, Materials Today 9 (2006) 36.

[8] T. Ohgi, D. Fujita, Surface Science 532 (2003) 294.

[9] D. Anselmetti, T. Richmond, A. Baratoff, G. Borer, M. Dreier, M. Bernasconi, H. J. Guntherodt, Europhysics Letters 25 (1994) 297.

[10] C. Schonenberger, H. Vanhouten, H. C. Donkersloot, Europhysics Letters 20 (1992) 249.

[11] J. C. Slonczewski, Physical Review B 39 (1989) 6995.

[12] U. Meirav, E. B. Foxman, Semiconductor Science and Technology 11 (1996) 255.

[13] R. P. Andres, T. Bein, M. Dorogi, S. Feng, J. I. Henderson, C. P. Kubiak, W. Mahoney, R. G. Osifchin, R. Reifenberger, Science 272 (1996) 1323. 
[14] S. Chen, R. W. Murray, The Journal of Physical Chemistry B 103 (1999) 9996.

[15] W. J. De Haas, J. De Boer, G. J. Van den Berg, Physica 2 (1935) 453.

[16] J. Kondo, Progress of Theoretical Physics 32 (1964) 37.

[17] P. W. Anderson, Physical Review 124 (1961) 41.

[18] G. Bergmann, Physical Review B 77 (2008)

[19] L. Kouwenhoven, L. Glazman, Physics World 14 (2001) 33.

[20] M. A. Ruderman, C. Kittel, Physical Review 96 (1954) 99.

[21] T. Kasuya, Progress of Theoretical Physics 16 (1956) 45.

[22] K. Yosida, Physical Review 106 (1957) 893.

[23] Y. Aharonov, D. Bohm, Physical Review 115 (1959) 485.

[24] R. A. Webb, S. Washburn, C. P. Umbach, R. B. Laibowitz, Physical Review Letters 54 (1985) 4.

[25] P. A. Lee, A. D. Stone, Physical Review Letters 55 (1985) 1622.

[26] G. Bergmann, Physical Review B 28 (1983) 2914.

[27] L. Van den dries, C. Van Haesendonck, Y. Bruynseraede, G. Deutscher, Physical Review Letters 46 (1981) 565.

[28] G. Bergmann, Physics Reports 107 (1984) 1.

[29] B. I. Altshuler, A. G. Aronov, Electron-electron interaction in disordered systems North Holland (1985) 


\section{Low-temperature solution synthesis of chemically functional ferromagnetic FePtAu nanoparticles}

Magnetic nanoparticles are of great scientific and technological interest. The application of ferromagnetic nanoparticles for high-density data storage has great potential, but energy efficient synthesis of uniform, isolated and patternable nanoparticles that remain ferromagnetic at room temperature, is not trivial. Here, we present a low-temperature solution synthesis method for FePtAu nanoparticles that addresses all those issues and therefore can be regarded as an important step towards applications. We show that the onset of the chemically ordered fct $\left(\mathrm{L}_{0}\right)$ phase is obtained for thermal annealing temperatures as low as $150{ }^{\circ} \mathrm{C}$. Large uniaxial magnetic anisotropy $\left(10^{7} \mathrm{erg} / \mathrm{cm}^{3}\right)$ and a high long-range order parameter have been obtained. Our low-temperature solution annealing leaves the organic ligands intact, so that the possibility for post-anneal monolayer formation and chemically assisted patterning on a surface is maintained.

This chapter has been published as S. Kinge, T. Gang, W.J.M. Naber, H. Boschker, G. Rijnders, D.N. Reinhoudt and W.G. van der Wiel, Nano Letters 9 (2009) 3220. 


\subsection{Introduction}

The continuously increasing demand for data storage capacity has very much stimulated research on magnetic recording media [1-2]. In modern hard disk drives, the magnetic medium layer is usually a CoCr-based alloy, containing sub-micron magnetic regions representing the bits of information. Every single magnetic region consists of $\sim 100$ magnetic grains, which are the basic elements to be magnetized. One of the main challenges in increasing the data storage capacity by reducing the magnetic grain size is maintaining its magnetization despite the superparamagnetic limit [3-6]. Current hard disk technology has an estimated limit of 1 terabit per square inch due to this superparamagnetic limit [1-2].

It has been argued [1-2] that thin layers (ideally monolayers) of ferromagnetic FePt nanoparticles (NPs) enable recording densities $\sim 10$ times larger than achievable with $\mathrm{CoCr}$-based media. Due to their very high magnetocrystalline anisotropy $\left(K_{u}=10^{7} \mathrm{erg} / \mathrm{cm}^{3}\right)$, FePt NPs remain ferromagnetic up to room temperature, even for few $\mathrm{nm}$ particle sizes. Furthermore, in traditional magnetic media, grain sizes show a wide distribution in size and shape, reducing the signal to noise ratio. In contrast, FePt NPs can be chemically synthesized with a highly uniform shape and narrow size distribution [1-2]. This ultimately allows for one bit per $\mathrm{nm}$-sized grain storage capacity and breaks the $1 \mathrm{~Tb}$ per square inch limit [7].

One of the major issues in FePt NP growth, however, is the need for a high-temperature annealing treatment $\left(\sim 700^{\circ} \mathrm{C}\right.$ and above) to obtain the desired high magnetocrystalline anisotropy [8]. The as-synthesized FePt NPs are namely in the chemically disordered face-centered-cubic (fcc) phase, which has low magnetic anisotropy. High-temperature annealing converts the NPs into the chemically ordered face-centered-tetragonal (fct) phase, referred to as the $\mathrm{LI}_{0}$ phase, where $\mathrm{Fe}$ and $\mathrm{Pt}$ planes alternate along the c-axis. High-temperature annealing, however, has a couple of severe disadvantages. Annealing is usually performed on dried nanopowders, which often results in particle agglomeration, and consequently a reduction of the particle uniformity and magnetic anisotropy. High-temperature annealing also destroys the organic ligands of the NPs, which 
takes away the advantage of the specific chemical functionality of the end groups, useful for chemical recognition and self-assembly in monolayers. A couple of methods have been developed to avoid agglomeration upon annealing, including thick $(10 \mathrm{~nm}) \mathrm{SiO}_{2}$ coating [9], salt matrix annealing [10], zeolite matrix annealing [11], and quite recently MgO coating [12-13]. Although these methods reduce agglomeration and result in ferromagnetic NPs at room temperature, still high temperatures are required and consequently the organic ligands are destroyed, losing all chemical functionality.

Given the above problems, a reduced annealing temperature is strongly favoured. Doping the FePt lattice with specific transition metals turns out to be advantageous for the $L 1_{0}$ phase transformation [1-2, 14]. Au (or Ag) doping in small amounts leads to significant lowering of the annealing temperature for transforming the fcc phase to the fct $\mathrm{L}_{0}$ phase. This is suggested to be related to defects and strain introduced by $\mathrm{Au}$ (or $\mathrm{Ag}$ ) atoms. Upon annealing, $\mathrm{Au}$ (or $\mathrm{Ag}$ ) atoms leave the FePt lattice at low temperature, leaving lattice vacancies that increase the mobility of Fe and Pt atoms to rearrange [3-6, 14]. Dry annealing studies of FePtAu NPs show a lowering of the annealing temperature with at least $100{ }^{\circ} \mathrm{C}$ compared to FePt NPs $[3-6,14]$. Although dry annealing at reduced annealing temperatures results in (partly) transformation into the $\mathrm{L}_{0}$ phase, still large-scale NP agglomeration occurs [15-17]. A way to avoid this is to anneal the NPs in a liquid. Harrell et al. investigated post-synthesis, high-pressure annealing of FePtAu NPs in diphenyl ether solvent, and in silicone oil at atmospheric pressure [14]. However, these methods result in significant increase in particle size. Alternatively, one can already perform the NP synthesis at elevated temperature in a high-boiling point solution. This was done by Jia et al., improving somewhat the dispersity [18].

In this chapter, we present a comprehensive and systematic study of low-temperature, solution synthesis that results in highly uniform ferromagnetic and chemically patternable FePtAu NPs. Magnetic analysis indicates a large $\mathrm{L}_{0}$ phase fraction, and that NPs of few $\mathrm{nm}$ size remain ferromagnetic up to room temperature. The onset for the $L 1_{0}$ phase occurs for annealing temperatures as low as $150{ }^{\circ} \mathrm{C}$, where the long-range order parameter $S$ [16] increases monotonically with annealing temperature. Importantly, we find that our 
procedure leaves the organic ligands intact, and demonstrate post-anneal chemically assisted monolayer patterning. We thus synergistically combine organic and inorganic (magnetic) materials, as well as bottom-up (self-assembly) and top-down fabrication methods, being main motivations for organic spintronics [19].

\subsection{Preparation of FePtAu nanoparticles}

Our FePtAu NP synthesis is partly based on that of Jia et al. [18], see Section 3.7. To synthesize FePtAu NPs, we use a combination of oleic acid and oleyl amine as stabilizing agent. The preparation is based on the reduction of platinum acetylacetonate and gold acetate by a diol and the decomposition of iron pentacarbonyl in high-temperature solutions. The octyl ether and hexadecylamine are used as solvents. Importantly, the addition of octyl ether as a solvent is different from the original method described by Jia et al., and is considered essential in our case. Hexadecylamine is solid, whereas octyl ether is liquid at room temperature. This allows the metal precursors already to dissolve at low temperature in the octyl ether before the hexadecylamine becomes liquid. We expect that our improved mixing conditions are responsible for the small size dispersity for our NPs.

\subsection{Structural characterization of FePtAu nanoparticles}

Figure 3.1 shows transmission electron microscope (TEM) images of $(\mathrm{FePt})_{85} \mathrm{Au}_{15}$ NPs synthesized at standard conditions ( $30 \mathrm{~min}$ at $150{ }^{\circ} \mathrm{C}$, Fig. 3.1a), and for $3 \mathrm{hrs}$ at $150{ }^{\circ} \mathrm{C}-350{ }^{\circ} \mathrm{C}$ (Figs. $3.1 \mathrm{~b}-3.1 \mathrm{e}$, respectively). The TEM analysis indicates regular NP assembly and small size dispersion, in particular for the lowest synthesis temperatures (Fig. 3.1f). 

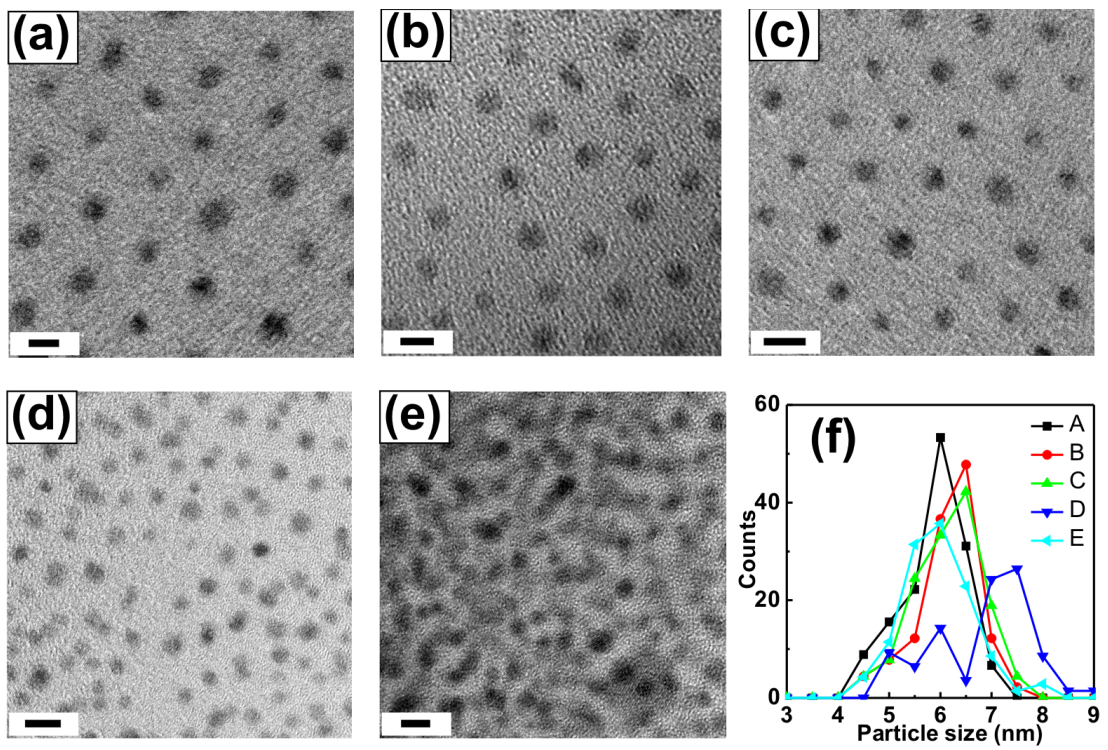

Figure 3.1: TEM images of $(\mathrm{FePt})_{85} \mathrm{Au}_{15}$ NPs synthesized under different conditions: (a) $150{ }^{\circ} \mathrm{C}, 30 \mathrm{~min}$; (b) $150{ }^{\circ} \mathrm{C}, 3 \mathrm{hrs}$; (c) $200{ }^{\circ} \mathrm{C}, 3 \mathrm{hrs}$; (d) $250{ }^{\circ} \mathrm{C}, 3 \mathrm{hrs}$; (e) $350{ }^{\circ} \mathrm{C}, 3 \mathrm{hrs}$. Scale bars correspond to $10 \mathrm{~nm}$. (f) Particle size distribution determined from TEM images. Curves A-E correspond to Figs. (a)-(e), respectively.

The particle diameters for different synthesis temperatures, derived from TEM analysis, and the elementary composition of the particles obtained from energy dispersive $\mathrm{X}$-ray diffraction (EDX) are given in Table 3.1. At $250{ }^{\circ} \mathrm{C}$ and $350^{\circ} \mathrm{C}$, the NPs have a broader size distribution. For synthesis at $150{ }^{\circ} \mathrm{C}$ and $200{ }^{\circ} \mathrm{C}$, the average NP composition is uniform and close to the metal precursor ratio. At $250{ }^{\circ} \mathrm{C}$ and $350{ }^{\circ} \mathrm{C}$ a relative large distribution of Au contents is observed. NPs with Au content as high as $\mathrm{Fe}_{16} \mathrm{Pt}_{22} \mathrm{Au}_{62}$ and as low as $\mathrm{Fe}_{47} \mathrm{Pt}_{45} \mathrm{Au}_{8}$ are observed for $250{ }^{\circ} \mathrm{C}$. For $350{ }^{\circ} \mathrm{C}$ the highest $\mathrm{Au}$ content was $\mathrm{Fe}_{21} \mathrm{Pt}_{24} \mathrm{Au}_{55}$ and lowest $\mathrm{Fe}_{41} \mathrm{Pt}_{47} \mathrm{Au}_{12}$. This suggests the segregation of $\mathrm{Au}$ atoms from the FePt bulk at higher temperatures. This is in agreement with the mechanism suggested above that Au creates empty sites, which can subsequently be occupied by randomly distributed Fe and Pt atoms, thereby transferring the fcc disordered phase into the ordered fct $\left(\mathrm{LI}_{0}\right)$ phase $[3-6,14]$. 
Table 3.1: Summary of TEM particle sizes and lattice parameters calculated from XRD analysis.

\begin{tabular}{|c|c|c|c|c|c|}
\hline sample & $\begin{array}{l}\text { synthesis } \\
\text { temp. } \\
\left({ }^{\circ} \mathrm{C}\right)\end{array}$ & $\begin{array}{l}\text { synthesis } \\
\text { time } \\
\text { (hrs) }\end{array}$ & $\begin{array}{l}\text { diameter } \\
\text { (nm) } \\
(\text { TEM) }\end{array}$ & $\begin{array}{l}\text { particle } \\
\text { composition } \\
\text { (EDX) }\end{array}$ & $\begin{array}{l}\text { lattice constant } \\
(\AA ̊) \quad(X R D) \\
{[111]}\end{array}$ \\
\hline$A$ & 150 & 0.5 & $5.5 \pm 0.3$ & $\mathrm{Fe}_{42} \mathrm{Pt}_{41} \mathrm{Au}_{17}$ & 2.294 \\
\hline B & 150 & 3 & $6.2 \pm 0.3$ & $\mathrm{Fe}_{42} \mathrm{Pt}_{44} \mathrm{Au}_{14}$ & 2.262 \\
\hline C & 200 & 3 & $6.4 \pm 0.3$ & $\mathrm{Fe}_{42} \mathrm{Pt}_{40} \mathrm{Au}_{18}$ & 2.241 \\
\hline D & 250 & 3 & $7.2 \pm 0.3$ & distributed & 2.234 \\
\hline$E$ & 350 & 3 & $5.8 \pm 2.3$ & distributed & 2.211 \\
\hline
\end{tabular}

Figure 3.2 shows the XRD analysis, indicating the evolution of the $L 1_{0}$ phase with increasing synthesis temperature. The evolution of the superlattice peaks, (001) and (110), as well as the fundamental peak (002), are clearly observed. The development of the $\mathrm{Au}$ (111) peak indicates the segregation of $\mathrm{Au}$ atoms from the FePt fcc lattice thereby transforming the lattice to fct $\left(\mathrm{LI}_{0}\right)$.

The lattice constant determined from the Pt (111) peak indicates a gradual decrease from $2.262 \AA$ ( $150{ }^{\circ} \mathrm{C}, 3 \mathrm{hrs}$ ), to $2.211 \AA$ ( $350{ }^{\circ} \mathrm{C}, 3 \mathrm{hrs}$ ), see Table 3.1, slowly approaching the ideal value of $2.197 \AA$ for a FePt fct lattice. The as-synthesized particles $\left(150{ }^{\circ} \mathrm{C}, 30 \mathrm{~min}\right)$ show a lattice constant of $2.294 \AA$, indicating a fcc lattice. This clearly demonstrates the phase transformation from fcc to fct NPs.

To quantify the chemical ordering in the $\mathrm{L}_{0}$ phase, one often uses the long-range ordering parameter $S$, defined as[20]

$S \cong 0.85\left(\frac{I_{001}}{I_{002}}\right)^{\frac{1}{2}}$,

where $I_{001}$ and $I_{002}$ are the integrated intensities of the superlattice 001 and fundamental 002 peaks in the XRD spectrum. $S$ is unity for perfectly ordered films and is zero for a chemically disordered film [21]. The long-range order parameter 
$S$ for different synthesis temperatures extracted from the XRD data is plotted in Fig. 3.3d (open symbols). The ordering parameter linearly increases with synthesis temperature, reaching $S=0.68$ for $350{ }^{\circ} \mathrm{C}$. For comparison, Sun et al. have achieved an ordering parameter of $S=0.98$ for dry annealing at $725^{\circ} \mathrm{C}$ for 2 hrs in $\mathrm{He}$ [1-2]. Here, we achieve significant ordering without much agglomeration at temperatures not more than $350^{\circ} \mathrm{C}$, maintaining the end group functionality (see below). The ordering process starts already from $150{ }^{\circ} \mathrm{C}$ onwards for longer synthesis times. This is very remarkable as previous reports for annealed NP powders showed the onset of ordering around $350{ }^{\circ} \mathrm{C}(30 \mathrm{~min})$ $[3-6,14]$.

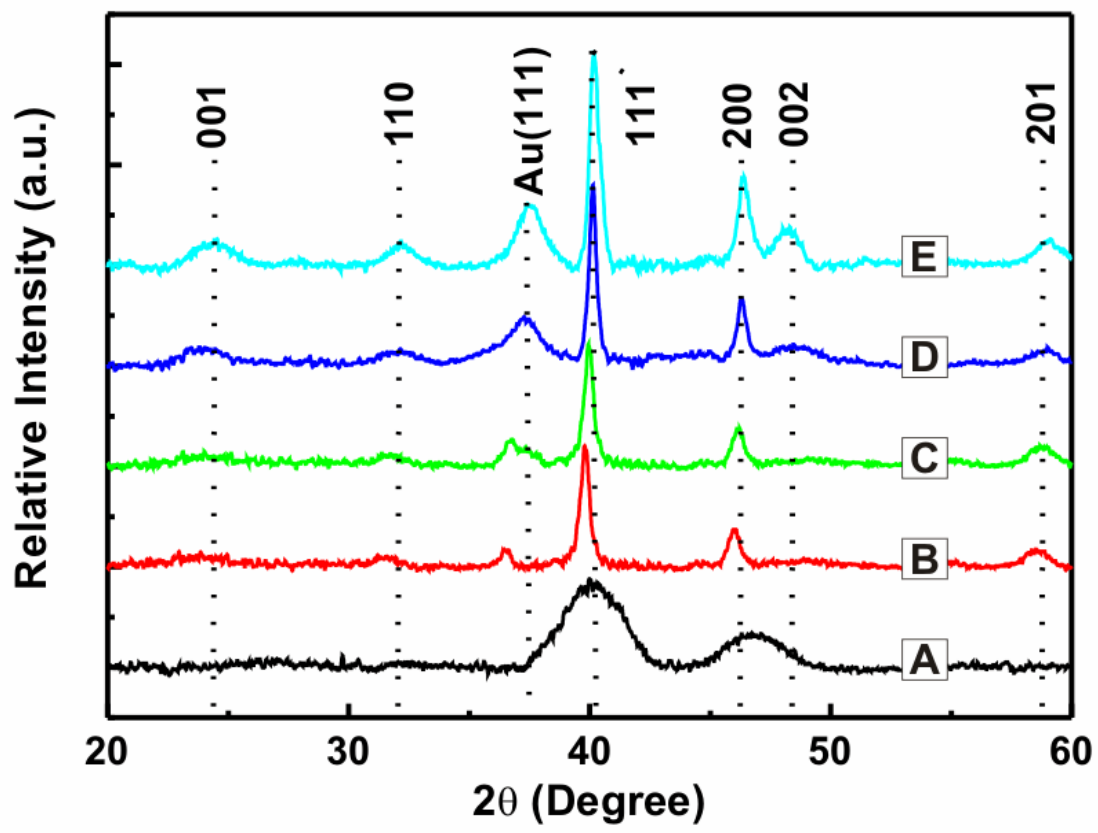

Figure 3.2: XRD spectra of (FePt) ${ }_{85} \mathrm{Au}_{15} \mathrm{NPs}, 150{ }^{\circ} \mathrm{C}, 30 \mathrm{~min}(\mathrm{~A}) ; 150{ }^{\circ} \mathrm{C}, 3 \mathrm{hrs}(\mathrm{B}) ; 200{ }^{\circ} \mathrm{C}, 3$ hrs (C); $250{ }^{\circ} \mathrm{C}, 3 \mathrm{hrs}(\mathrm{D}) ; 350^{\circ} \mathrm{C}, 3 \mathrm{hrs}(\mathrm{E})$.

As suggested by Chepulskii et al., the ordering process is kinetically regulated [17, 22]. As a consequence, it is expected that approaching the equilibrium ordered state at low temperature will take longer. Therefore kinetic acceleration methods such as irradiation and/or addition of other types of atoms are potentially 
effective in accelerating the formation of long-range order. To the best of our knowledge, all the previous studies report the synthesis of NPs by refluxing the precursor's solution only for $30 \mathrm{~min}$. Here, we have increased the duration of gentle refluxing to $3 \mathrm{hrs}$. Increasing the refluxing time gives the NPs more time to organize in the desired crystal phase (kinetic control). This results in significantly higher chemical ordering of our NPs in the fct phase.

X-ray photoelectron spectroscopy (XPS) is performed to analyze the elemental states of $\mathrm{Fe}, \mathrm{Pt}$ and $\mathrm{Au}$ in the NPs. The NP surface composition indicates the presence of $\mathrm{Fe}_{2} \mathrm{O}_{3}$, while removal of the outer $1 \mathrm{~nm}$ shell by sputtering results in a composition of elements near to the expected value. This suggests that the surface is partially covered with $\mathrm{Fe}_{2} \mathrm{O}_{3}$.

\subsection{Magnetic properties of FePtAu nanoparticles}

We have prepared monolayers of FePtAu NPs for magnetic characterization [2, 21]. A silicon/silicon oxide substrate is functionalized with a very thin polyethylenimine (PEI) layer. By dipping the polymer-derivative substrate into the particle dispersion, pendant functional groups of the polymer replace the particle stabilizers and a strong monolayer particle assembly is formed. The substrate is then rinsed with solvent to remove physical sorption and dried. This process results in one single FePtAu NP monolayer.

The magnetic properties of NP monolayers are characterized by a vibrating sample magnetometer (VSM) with variable temperature insert (5-300 K). The magnetization curves at $5 \mathrm{~K}$ and $300 \mathrm{~K}$ for NPs synthesized at different temperatures are shown in Figs. 3.3a and 3.3b, respectively. The coercive field $\left(H_{c}\right)$ clearly increases with synthesis temperature. At $5 \mathrm{~K}$ magnetic hysteresis is observed for all synthesis temperatures, whereas at $300 \mathrm{~K}$ hysteresis is observed for synthesis temperatures $200{ }^{\circ} \mathrm{C}\left(H_{\mathrm{c}}=600 \mathrm{Oe}\right), \quad 250{ }^{\circ} \mathrm{C} \quad\left(H_{\mathrm{c}}=2700 \mathrm{Oe}\right)$ and $350{ }^{\circ} \mathrm{C}\left(H_{\mathrm{c}}=4800 \mathrm{Oe}\right)$. This demonstrates room-temperature ferromagnetism from syntesis temperatures starting from $200^{\circ} \mathrm{C}$ only. 

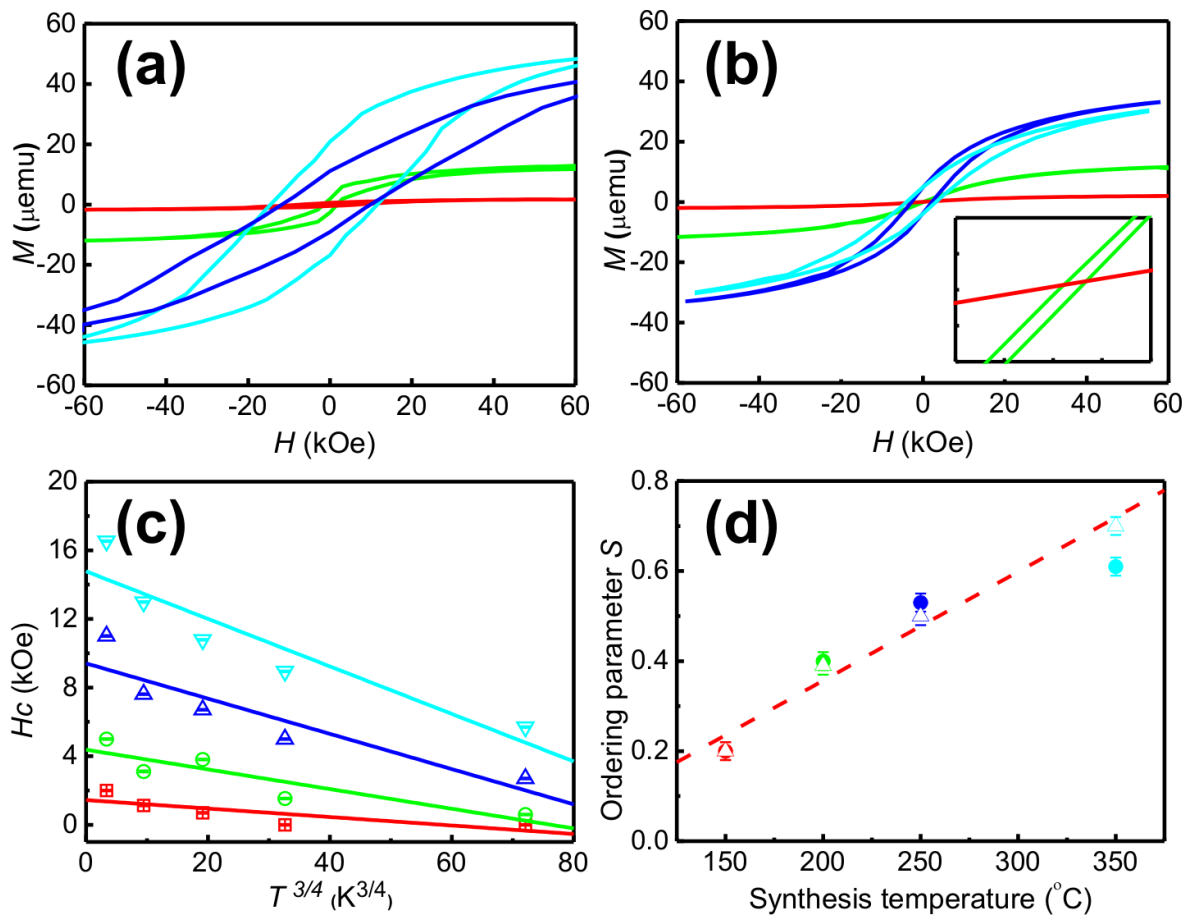

Figure 3.3: NP magnetization curves for different synthesis conditions measured by VSM at $5 \mathrm{~K}(\mathrm{a})$ and at room temperature (b). The inset of (b) is a zoom in around zero field. The horizontal and vertical axis range is $0.5 \mathrm{kOe}$ and $0.2 \mu \mathrm{emu}$, respectively. In all figures, the curve color refers to the different synthesis conditions: red $150{ }^{\circ} \mathrm{C}$, 3hrs; green $200{ }^{\circ} \mathrm{C}, 3$ hrs; blue $250{ }^{\circ} \mathrm{C}, 3 \mathrm{hrs}$; and cyan $350^{\circ} \mathrm{C}, 3 \mathrm{hrs}$. $H_{\mathrm{c}}$ versus $T^{3 / 4}$ plots, used for fitting to the Garcia-Otero model, see text (c). Ordering parameter $S$, extracted from XRD data (open triangles) and $H_{\mathrm{c}}$ versus $T^{3 / 4}$ plots (solid dots), respectively, versus synthesis temperature (d). The linear dashed line is a guide to the eye.

The saturation magnetic moment of our monolayers is $\sim 35-40 \mu \mathrm{emu}$, both at room temperature and low temperature. We compare this value for $350{ }^{\circ} \mathrm{C}$ synthesis temperature with the momentum density of bulk FePt $\left(1140 \mathrm{emu} / \mathrm{cm}^{3}\right)$ [23], assuming the ratio of FePt in our FePtAu NPs to be $0.85,2.9 \mathrm{~nm}$ NP radius and $1 \mathrm{~nm}$ ligand length. Based on those parameters, we find (see Section 3.7) a saturation magnetic moment of $47 \mu \mathrm{emu}$. The experimentally observed value is slightly lower, as expected when taking into account the not fully developed $\mathrm{L}_{0}$ 
phase and lower effective packing density.

The observed temperature dependence of our magnetic hysteresis loops can be understood by realizing that the NP coercive fields decrease due to thermal fluctuations at the temperature increases. In this regard, Sharrock's formula [24] is the most widely used thermal relaxation model. However, it is valid for $2 \mathrm{D}$ systems rather than 3D systems like our NPs. Therefore, the model proposed by Garcia-Otero et al. is applied here to study the magnetic properties of NPs. This model applies to magnetically isolated and 3D random particle systems [20]. It can be described as

$\frac{H_{c}}{H_{k}}=0.479-0.81\left(\frac{k_{b} T}{2 K_{u} V}\left(\ln \tau_{m}+20.7\right)\right)^{\frac{3}{4}}$,

where $H_{k}$ is the critical field in Oe, defined as the field at which the NPs undergo an irreversible jump in their magnetization direction when decreasing the magnetic field, $k_{B}$ the Boltzmann constant $\left(1.38073 \times 10^{-16} \mathrm{erg} / \mathrm{K}\right), K_{u}$ is the uniaxial anisotropy energy density in $\mathrm{erg} / \mathrm{cm}^{3}, V$ is the particle volume in $\mathrm{cm}^{3}$, and $\tau_{m}$ is the measurement time in seconds. Using this model, the magnetic properties of the magnetic NPs can be evaluated. We have plotted $H_{c}$ vs. $T^{3 / 4}$ in Fig. 3.3c. According to Eq. (3.2), from the intercept of the $H_{c}$ versus $T^{3 / 4}$ curves we can derive $H_{k}$. From the slopes of the curves $K_{u} V$ is derived.

The anisotropy constants derived from the fits with Eq. (3.2) in Fig. 3.3c are given in Table 3.2. The long-range ordering parameter $S$ for different annealing temperatures obtained based on a measured relationship between anisotropy constants $K_{u}$ and ordering parameter $S$, is given in Table 3.2 as well, and plotted in Fig. 3.3d (solid symbols) [25]. The obtained ordering parameter is in good agreement with the ordering parameter determined by XRD (open symbols in Fig. 3.3d). A large anisotropy constant underlines the potential of these NPs for high-density data storage. 
Table 3.2: Anisotropy constant $K_{\mathrm{u}}$ derived from Garcia-Otero model [20] and corresponding long-range ordering parameter $S$.

\begin{tabular}{lllll}
\hline sample & $\begin{array}{l}\text { synthesis } \\
\text { temp. } \\
\left({ }^{\circ} \mathrm{C}\right)\end{array}$ & $\begin{array}{l}\text { synthesis } \\
\text { time } \\
(\mathrm{hrs})\end{array}$ & $\begin{array}{l}\text { anisotropy } \\
\text { constant } K_{\mathrm{u}} \\
\left(10^{7} \mathrm{erg} / \mathrm{cc}\right)\end{array}$ & $\begin{array}{l}\text { ordering } \\
\text { parameter } S \\
\left(\text { derived from } K_{u}\right)\end{array}$ \\
\hline B & 150 & 3 & 1.2 & 0.2 \\
C & 200 & 3 & 2.0 & 0.4 \\
D & 250 & 3 & 2.3 & 0.5 \\
E & 350 & 3 & 2.7 & 0.6 \\
\hline
\end{tabular}

\subsection{Patterning of FePtAu nanoparticles}

Solution annealed FePtAu NPs disperse very well, indicating that the ligands are still intact. In order to demonstrate that post-anneal patterning based on ligand exchange is still possible with our NPs, we have fabricated the NP patterns of Fig. 3.4. The $\mathrm{Si} / \mathrm{SiO}_{2}$ substrate is covered with a thin $(0.62 \pm 0.04 \mathrm{~nm})$ of hexamethyldisilazane (HMDS). Subsequently a pattern is defined by standard photolithography for selective self-assembly of the FePtAu NPs. When dipping the substrate in the particle dispersion, pendant functional groups $-\mathrm{NH}$ - of the HMDS replace the particle stabilizers and a strong NP monolayer is formed in the exposed regions. Physisorbed NPs and resist are easily removed afterward. The successful patterning of the FePtAu NPs based on ligand exchange indicates that the ligands are intact after our solution annealing procedure. 


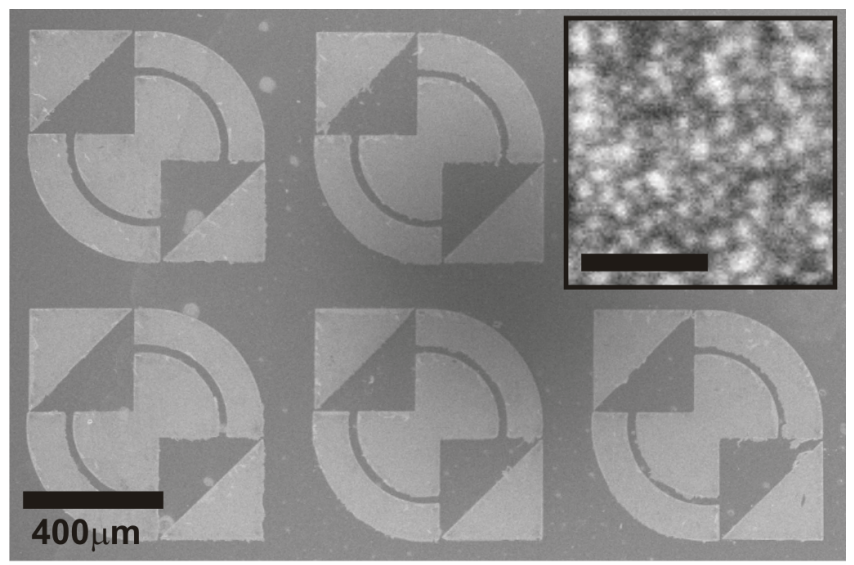

Figure 3.4: Scanning electron microscope (SEM) images of FePtAu NPs monolayer patterns. The insert shows a zoom in on the patterned area. The scale bar is $50 \mathrm{~nm}$.

\subsection{Conclusion}

In conclusion, we have demonstrated a low-temperature solution synthesis method for fabricating FePtAu nanoparticles that remain ferromagnetic up to room temperature. Our method strongly reduces nanoparticle agglomeration, associated with high annealing temperatures and leaves the organic ligands intact. As a result, the post-annealed nanoparticles still leave the flexibility for further processing, patterning for instance. Low-temperature bottom-up synthesis of ferromagnetic, patternable nanoparticles is considered to be an important step towards application of ferromagnetic particles in high-density data storage and spintronics.

\subsection{Experimental section}

The NPs were prepared using a modification of the reported procedure by Jia et al. [18]. The synthetic experiments were carried out using standard airless procedures and commercially available reagents. The syntheses were performed by heating the precursors' solution at different temperatures $150{ }^{\circ} \mathrm{C}, 200{ }^{\circ} \mathrm{C}$, $250{ }^{\circ} \mathrm{C}, 350^{\circ} \mathrm{C}$ for $3 \mathrm{hr}$. At first, a solution of platinum acetylacetonate $(0.5 \mathrm{mmol})$, 
gold acetate $(0.05-0.35 \mathrm{mmol})$, and 1,2-hexadecanediol $(1.5 \mathrm{mmol})$ in $20-\mathrm{mL}$ octyl ether and $20 \mathrm{~mL}$ hexadecylamine was heated up to $100^{\circ} \mathrm{C}$ in a three-necked, round-bottom flask under a nitrogen atmosphere. To this solution was added, via syringe, oleic acid $(0.5 \mathrm{mmol})$, oleylamine $(0.5 \mathrm{mmol})$, and iron pentacarbonyl (1 $\mathrm{mmol})$. The mixture was heated to reflux and allowed to reflux for $30 \mathrm{~min}$ or $3 \mathrm{hr}$ resulting in a black dispersion. Then the heat source was removed and the dispersion was allowed to cool to the room temperature. The inert gas protected solution could then be opened to ambient environment. Adding $40-\mathrm{mL}$ ethanol precipitated the black product. The mixture was centrifuged to isolate the particles from the brown supernatant. The particles were redispersed in hexane, precipitated with ethanol, and isolated by centrifuging. The particles were dried at room temperature in a vacuum oven to give 100-200 mg of particles. The dispersion and precipitation removed impurities. During synthesis, the relative amounts of platinum acetylacetonate and iron pentacarbonyl and gold acetate were fixed in order to produce NPs with similar compositions $(\mathrm{FePt})_{85} \mathrm{Au}_{15}$.

FePtAu NPs monolayers were prepared via polymer mediate self-assmbely [9, 18]. Oxygen plasma treated silicon substrates were immersed in $20 \mathrm{mg} / \mathrm{ml}$ chloroform solution of PEI for $5 \mathrm{~min}$ and then dipped in ethanol several times to wash away extra PEI. PEI covered silicon substrates were immersed into FePt $(10 \mathrm{mg} / \mathrm{ml})$ hexane solution for $10 \mathrm{~min}$ to assemble FePtAu NPs on the surfaces. Then the substrates were rinsed with pure hexane to wash off physisorbed particles and subsequently dried under $\mathrm{N}_{2}$ flow.

FePtAu NPs monolayer patterns are prepared as flows. Patterns on the photoresist film were prepared using Hexamethyldisilazane (HMDS) modified silicon substrate via standard photolithography. The lithographic defined substrate was immersed into FePt $(10 \mathrm{mg} / \mathrm{ml})$ hexane solution for $10 \mathrm{~min}$ to assemble FePtAu NPs on the surfaces. Then the substrates were rinsed with pure hexane to wash off physisorbed particles. Finally, the substrate was rinsed thoroughly with acetone until all the photo resist was removed.

TEM images of NPs were recorded with a Philips CM-30 Twin operating at $200 \mathrm{kV}$. A drop of hexane solution of the NPs was deposited on the carbon-coated copper grid and allowing the solvent to slowly evaporate. The average NP size was 
determined averaging over 150 NPs from different frames of TEM images.

The NP sample was analyzed by powder X-ray diffraction (XRD) analysis using a Philips X'Pert diffractometer (CuK ${ }_{\alpha} \lambda=1.5418 \AA$ ).

XPS measurements were performed with Physical Electronics Quantum 2000 equipment. It is equipped with a spherical sector analyzer and a multi-channel plate detector. Analyzer Mode was constant pass energy. For the survey scan the pass energy was $117 \mathrm{eV}$, the X-ray beam was set to high-power mode 100 Watt/100 $\mu \mathrm{m}$, and the diameter beam scanned over a $1000 \mu \mathrm{m} \times 500 \mu \mathrm{m}$ area. For element scans pass energy was $29.35 \mathrm{eV}$, the X-ray beam was set to 25 $\mathrm{W} / 100 \mu \mathrm{m}$, and the diameter beam scanned over a $1000 \mu \mathrm{m} \times 500 \mu \mathrm{m}$ area. The excitation source was $\mathrm{AlK}_{\alpha}$ monochromatic radiation with a source energy = $1486.6 \mathrm{eV}$. The take-off angle (analyzer angle to sample surface) was set to 30 degrees. Using both low energy electrons and ions controlled the surface potential. The temperature during analysis was $298 \mathrm{~K}$, and the pressure was between 1 and $3 \times 10^{-8}$ Torr (Argon pressure for charge control). For atomic concentration calculation, the Shirley background subtraction was employed. The sensitivity factors were provided by Physical Electronics Multipack software version $6.1 \mathrm{~A}$. The hydrocarbon $\mathrm{C} 1 \mathrm{~s}$ signal at $284.8 \mathrm{eV}$ was used as a reference for surface charging.

Magnetic hysteresis curves were recorded in a Quantum Design vibrating sample magnetometer (VSM). This gives information about the phase transformation and ferromagnetic behavior with field up to $9 \mathrm{~T}$ and temperature between $5 \mathrm{~K}$ and $300 \mathrm{~K}$.

The theoretical magnetic momentum was calculated via equation 3.3. In the equation, $m$ is the magnetic momentum, $M$ is the momentum density, $n$ is number of NPs on the substrate, $S$ is the area of substrate, $\rho$ is the packing density, $r_{1}$ is the radius of NP, $r_{2}$ is the length of the ligands and $p$ is the ratio of FePt in FePtAu NPs.

$m=M n V=M \frac{s \rho}{\pi\left(r_{1}+r_{2}\right)^{2}}\left(\frac{4}{3} \pi r_{1}^{3}\right) p$,

Momentum density of bulk FePt is 1140 emu/cc [23]. The substrate dimension is about $2.5 \mathrm{~mm}$ by $10 \mathrm{~mm}$ and the ratio of FePt in FePtAu NPs is 0.85 . Assuming a 
hexagonal packing of NPs on substrates $(\rho=0.9069)$, the length of the ligands is estimated to be $1 \mathrm{~nm}$. In case of FePtAu NPs annealed at $350{ }^{\circ} \mathrm{C}$, radius of NP is $2.9 \mathrm{~nm}$ according to TEM analysis.

\section{References}

[1] S. H. Sun, Advanced Materials 18 (2006) 393.

[2] S. H. Sun, C. B. Murray, D. Weller, L. Folks, A. Moser, Science 287 (2000) 1989.

[3] S. S. Kang, Z. Y. Jia, D. E. Nikles, J. W. Harrell, IEEE Transactions on Magnetics 39 (2003) 2753.

[4] S. H. Kang, Z. Y. Jia, D. E. Nikles, J. W. Harrell, IEEE Transactions on Magnetics 40 (2004) 513.

[5] S. Wang, S. S. Kang, D. E. Nikles, J. W. Harrell, X. W. Wu, Journal of Magnetism and Magnetic Materials 266 (2003) 49.

[6] C. H. Yu, N. Caiulo, C. C. H. Lo, K. Tam, S. C. Tsang, Advanced Materials 18 (2006) 2312.

[7] T. Schrefl, G. Hrkac, D. Suess, W. Scholz, J. Fidler, Journal of Applied Physics 93 (2003) 7041.

[8] I. Zafiropoulou, V. Tzitzios, D. Petridis, E. Devlin, J. Fidler, S. Hoefinger, D. Niarchos, Nanotechnology 16 (2005) 1603.

[9] S. Yamamoto, Y. Morimoto, T. Ono, M. Takano, Applied Physics Letters 87 (2005) 032503.

[10] B. A. Jones, J. D. Dutson, K. O'Grady, B. J. Hickey, D. R. Li, N. Poudyal, J. P. Liu, IEEE Transactions on Magnetics 42 (2006) 3066.

[11] S. Momose, H. Kodama, W. Yamagishi, T. Uzumaki, Japanese Journal of Applied Physics Part 2-Letters \& Express Letters 46 (2007) L1105.

[12] J. Kim, C. B. Rong, Y. Lee, J. P. Liu, S. H. Sun, Chemistry of Materials 20 (2008) 7242. 
[13] J. M. Kim, C. B. Rong, J. P. Liu, S. H. Sun, Advanced Materials 21 (2009) 906.

[14] J. W. Harrell, D. E. Nikles, S. S. Kang, X. C. Sun, Z. Jia, S. Shi, J. Lawson, G. B. Thompson, C. Srivastava, N. V. Seetala, Scripta Materialia 53 (2005) 411.

[15] H. Borchert, E. V. Shevehenko, A. Robert, I. Mekis, A. Kornowski, G. Grubel, H. Weller, Langmuir 21 (2005) 1931.

[16] B. E. Warren, X-Ray Diffraction Addison-Wesley Publishing Company (1969)

[17] R. V. Chepulskii, J. Velev, W. H. Butler, Journal of Applied Physics 97 (2005) $10 \mathrm{~J} 311$.

[18] Z. Y. Jia, S. S. Kang, D. E. Nikles, J. W. Harrell, IEEE Transactions on Magnetics 41 (2005) 3385.

[19] W. J. M. Naber, S. Faez, W. G. van der Wiel, Journal of Physics D-Applied Physics 40 (2007) R205.

[20] J. Garcia-Otero, A. J. Garcia-Bastida, J. Rivas, Journal of Magnetism and Magnetic Materials 189 (1998) 377.

[21] S. H. Sun, S. Anders, T. Thomson, J. E. E. Baglin, M. F. Toney, H. F. Hamann, C. B. Murray, B. D. Terris, Journal of Physical Chemistry B 107 (2003) 5419.

[22] R. V. Chepulskii, W. H. Butler, Physical Review B 72 (2005) 134205.

[23] T. Klemmer, D. Hoydick, H. Okumura, B. Zhang, W. A. Soffa, Scripta Metallurgica et Materialia 33 (1995) 1793.

[24] D. Weller, A. Moser, IEEE Transactions on Magnetics 35 (1999) 4423.

[25] T. Shima, T. Moriguchi, S. Mitani, K. Takanashi, Applied Physics Letters 80 (2002) 288. 


\section{Chapter 4}

\section{Magnetic nanoparticle assembly on surfaces using click-chemistry}

Controlled assembly of ferromagnetic nanoparticles on surfaces is of crucial importance for a range of spintronic and data storage applications. Here, we present a novel method for assembling monolayers of ferromagnetic FePt nanoparticles on silicon oxide substrates using "click-chemistry". Reaction of alkyne-functionalized FePt nanoparticles with azide-terminated self-assembled monolayers (SAMs), on silicon oxide, leads to the irreversible attachment of magnetic nanoparticles to the surface via triazole linkers. Based on this covalent interaction, well-packed monolayers of FePt nanoparticles were prepared and nanoparticle patterns are generated on surfaces via micro-contact printing ( $\mu \mathrm{CP})$.

This chapter has been published as S. Kinge, T. Gang, W. J. M. Naber, W. G. van der Wiel, D. N. Reinhoudt, Langmuir 27 (2010) 570. 


\subsection{Introduction}

Ferromagnetic nanoparticles are very promising for creating ultra-small devices for spintronics, magnetic sensing, and ultra-high density data storage [1-5]. In this regard monodisperse FePt nanoparticles are very interesting, and their synthesis and characteristics are extensively studied [1, 6-9]. After annealing, they show ferromagnetic behavior up to room temperature due to their very high magnetocrystalline anisotropy $\left(\mathrm{K}_{\mathrm{u}}=7 \times 10^{6} \mathrm{~J} / \mathrm{m}^{3}\right)$. In addition, they have higher chemical stability compared to Co-based data storage materials [8-10]. Instead of multiple grains for the storage of one bit of information, FePt nanoparticles allow for one bit per grain, enabling terabit/inch ${ }^{2}$ storage capacity [10]. However, for practical applications, a regularly assembled nanoparticle array is a prerequisite. Self-assembly of nanoparticles on surfaces is very attractive for this purpose. So far, only a few self-assembly methods have been reported to organize magnetic nanoparticles, like drying-mediated assembly, layer-by-layer assembly via electrostatic interactions and the coupling via amino terminated APTS [3-(2-aminoethlyamino)propyl]trimethoxysilane, which replaces the surfactants that stabilize the nanoparticles [11-15]. These methods have several drawbacks, such as instability or non-specific particle-surface interactions.

In this chapter, we present "click chemistry" as an alternative method to assemble monolayers of FePt nanoparticles via specific nanoparticle-substrate interactions. Click-chemistry is a very useful tool for creating new materials based on highly selective and quantitative coupling reactions, in particular the Huisgen 1,3 dipolar cycloaddition of azides and alkynes to form 1,2,3 triazoles [16-23]. The copper (I) catalyzed azide-alkyne cycloaddition reactions proceed with high yields and without byproducts [24-25]. This method has been used for variety of applications such as functionalization of polymers, biomolecules, bulk surfaces, etc [17-26]. Previously, Fleming et al.[27], White et al.[28], Lin et al. [29] and Binder et al.[30] have reported the functionalization of gold, iron oxide or CdSe nanoparticles using click-chemistry. Rozkiewica et al. in our collaborating group have exploited this approach to create molecular nanostructures on surfaces [31]. To the best of our knowledge, this is the first example of assembling and attaching nanoparticles on a surface using click-chemistry. 


\subsection{Assembly and patterning of FePt nanoparticles}

The FePt nanoparticles were synthesized according to Sun's method [14]. Subsequently, the oleic acid and oleyl amine groups at the surface of the nanoparticles were replaced by 5-hexanoic acid and 6-amino-1-hexyne groups to form alkyne-substituted FePt nanoparticles. These nanoparticles were assembled (Scheme 4.1) or patterned (Scheme 4.2) on the substrate by reaction with azide-terminated SAMs on silicon oxide substrates.

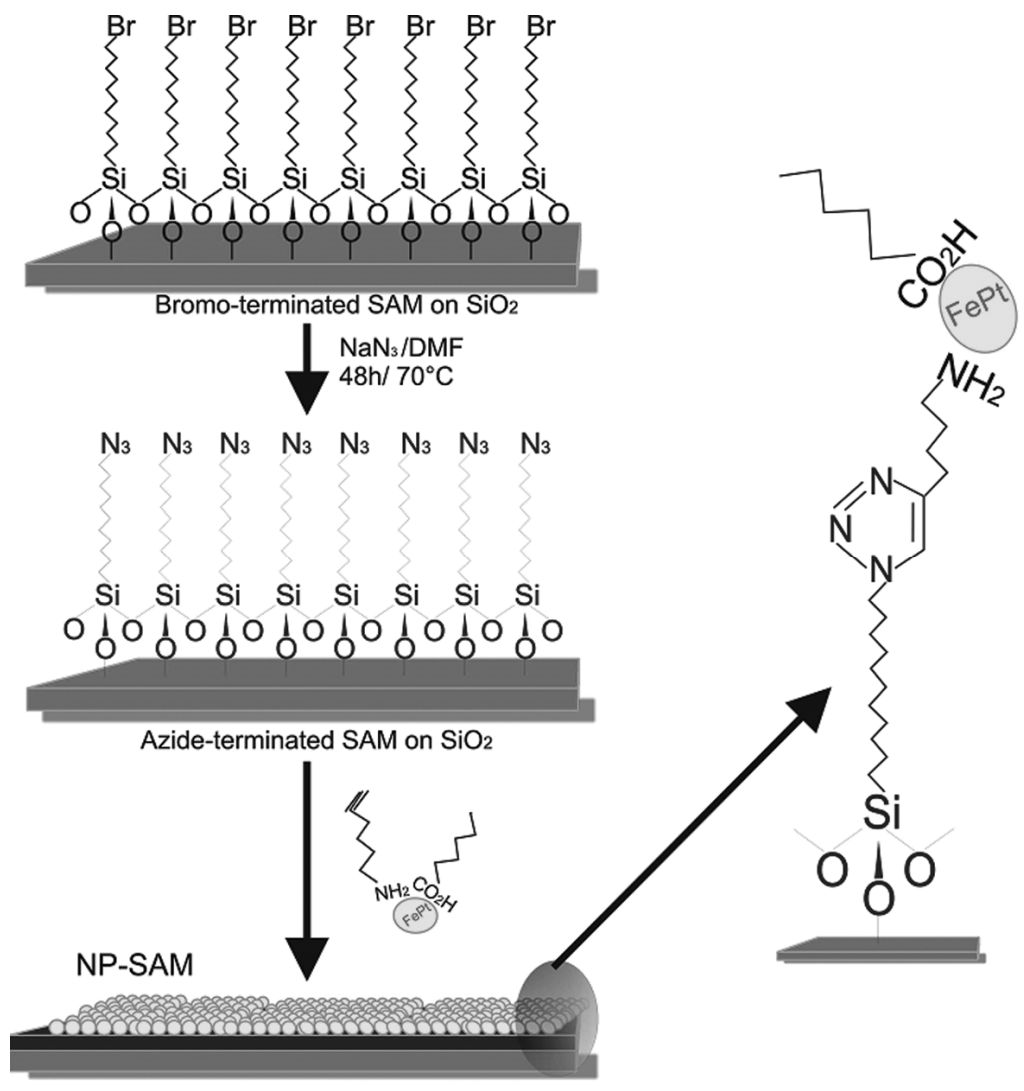

Scheme 4.1: Assembling ferromagnetic FePt nanoparticles as monolayer using click-chemistry. 


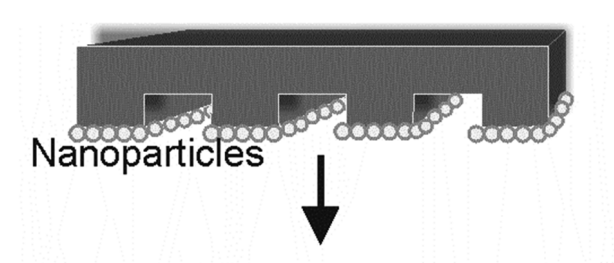

Azide SAM

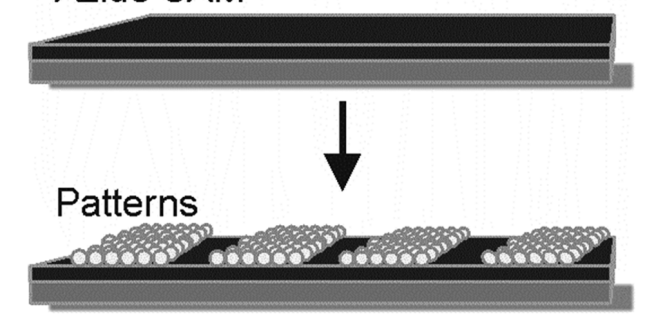

Scheme 4.2: Patterning ferromagnetic FePt nanoparticles via $\mu \mathrm{CP}$ based on click-chemistry.

Transmission electron microscopy (TEM) analysis of FePt nanoparticles functionalized with 6-amino 1-hexyne and 5-hexanoic acid indicated monodisperse nanoparticles of $5 \pm 0.5 \mathrm{~nm}$ size (Fig. 4.1). These nanoparticles were redispersed in hexane. Based on energy-dispersive X-ray spectroscopy (EDX) analysis the elemental composition was $\mathrm{Fe}_{48} \mathrm{Pt}_{52}$.
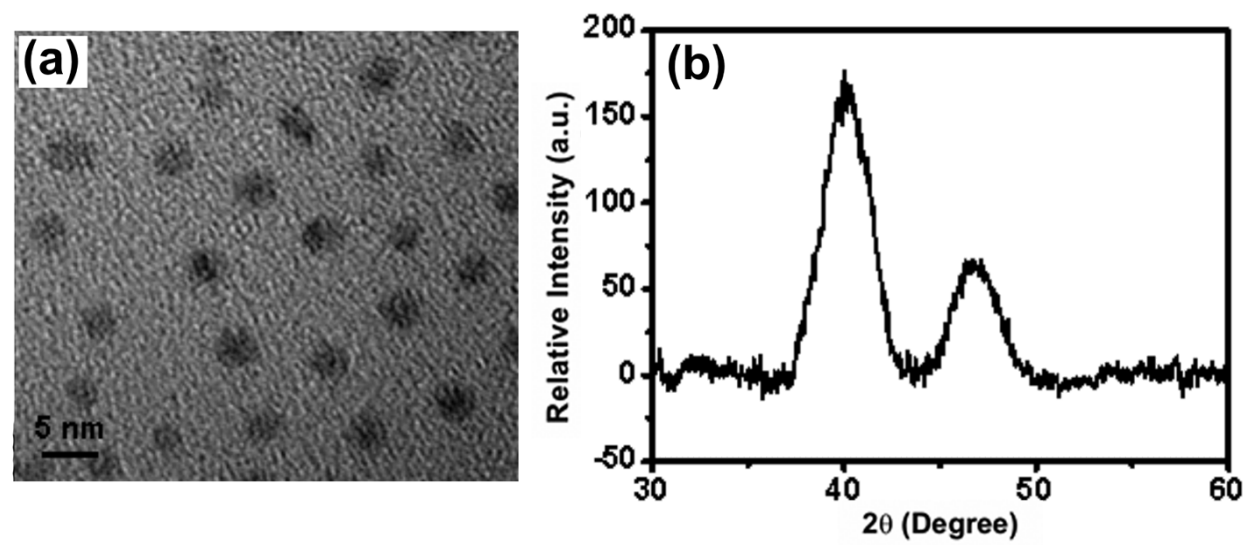

Figure 4.1: TEM micrograph of $5 \pm 0.5 \mathrm{~nm} \mathrm{Fe}{ }_{52} \mathrm{Pt}_{48}$ nanoparticles stabilized with 6-amino 1-hexyne and 5-hexanoic acid (a) and XRD pattern of the as-synthesized nanoparticles (b). 
Azide terminated SAMs were prepared in situ from 11- bromo-undecylsiloxane SAMs, to assemble or to create FePt nanoparticle patterns on the silicon oxide substrates. The azide terminated SAMs were subsequently reacted with alkyne terminated FePt nanoparticles according to Scheme 4.1. The exchange of the surface bromine group of 11-bromoundecylsiloxane SAMs against azide via nucleophilic substitution with DMF solution of sodium azide proceeded quantitatively and was accompanied in the infrared spectra by an intense $v\left(N_{3}\right)$ absorption at $2014 \mathrm{~cm}^{-1}$ corresponding to the asymmetric stretch (Fig. 4.2). The XPS spectrum of the bromo-terminated SAMs shows a pronounced $\operatorname{Br}(3 \mathrm{~d})$ peak at $70.5 \mathrm{eV}$ (Fig. 4.2). After substitution with $\mathrm{NaN}_{3}$, the $\mathrm{Br}(3 \mathrm{~d}$ ) peak disappeared and an $\mathrm{N}(1 \mathrm{~s})$ peak appeared at $400 \mathrm{eV}$, which confirmed complete reaction. Ellipsometry showed that the bromo SAM has a thickness $d=1.34 \pm 0.08 \mathrm{~nm}$ and the azido SAM $d=1.48 \pm 0.10 \mathrm{~nm}$, in agreement with the previous reported values $[22,30]$.

To create a monolayer of FePt nanoparticles on the silicon oxide substrate, the azide-terminated substrates were immersed in the alkyne-functionalized nanoparticle solution (DMSO: $\mathrm{H}_{2} \mathrm{O}=4: 1 \mathrm{v} / \mathrm{v}$ ) for $15 \mathrm{~min}$. $\mathrm{CuSO}_{4} 5 \mathrm{H}_{2} \mathrm{O}$ and sodium ascorbate were subsequently added to the solution to catalyze the reaction. Then the mixture was stirred for $48 \mathrm{hrs}$ at room temperature (RT) [30]. The substrates were removed and washed with ethanol to remove any physisorbed layer of nanoparticles.

The $\mu \mathrm{CP}$ was carried out with and without $\mathrm{Cu}(\mathrm{I})$ catalysts, and no significant differences were observed. As we have previously reported, during $\mu \mathrm{CP}$ a high local concentration of functional groups can give a click reaction without the need for catalysts [30]. To discriminate whether the particles are attached to the surface through physisorption or specific covalent reactions, a similar nanoparticle deposition was repeated on a bare substrate. In addition, non-alkyne functionalized FePt nanoparticles (oleic acid and oleyl amine stabilized) were also deposited on the azide-terminated substrates. The absence of nanoparticles in both cases clearly confirms that nanoparticles are attached through the triazole formation between the alkyne functionalized nanoparticles and azide-terminated substrate. 

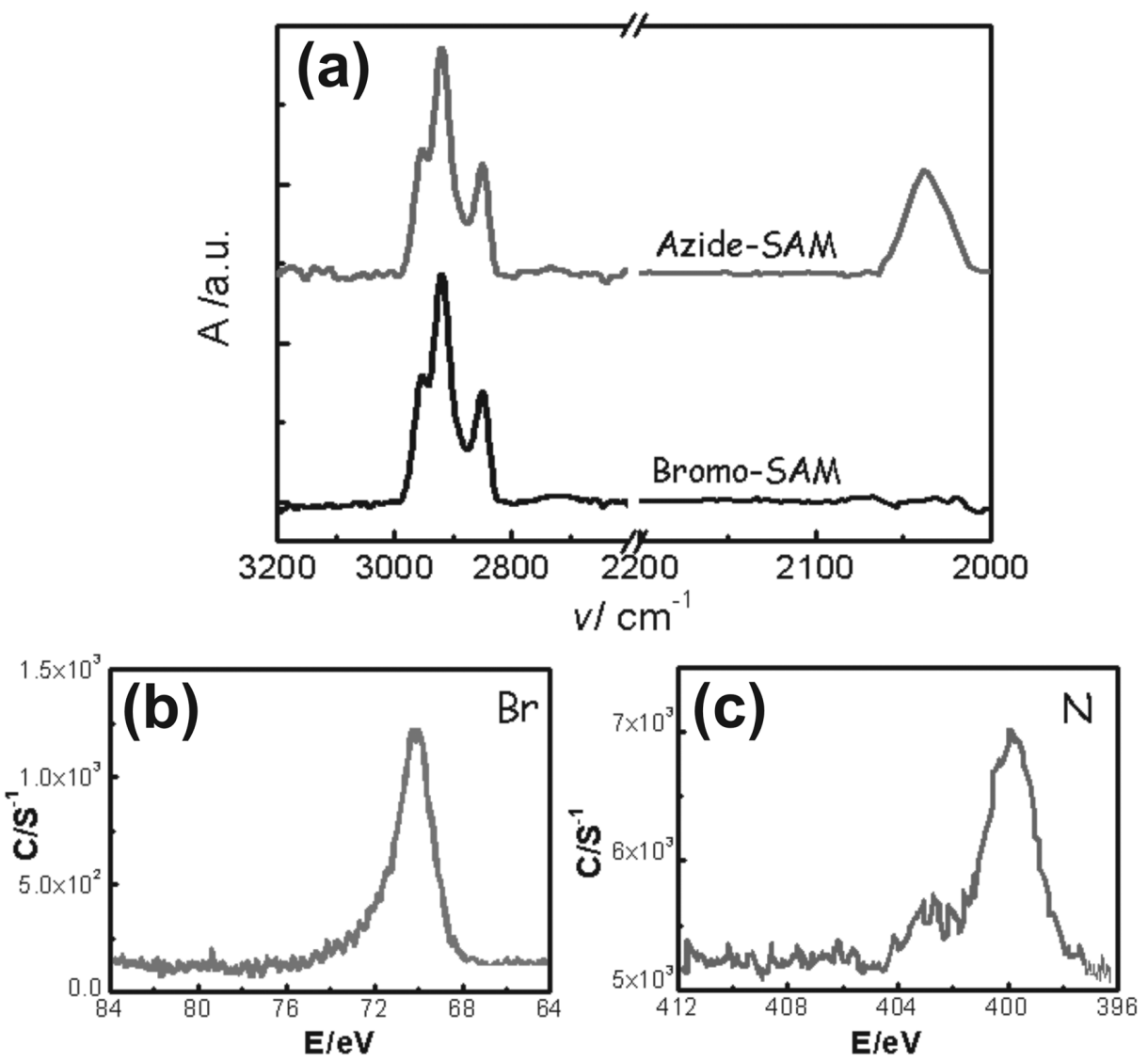

Figure 4.2: Fourier transform infrared spectroscopy (FTIR) spectra of bromo-terminated and azide-terminated SAMs on the silicon oxide substrates (a); XPS analysis showing the presence of $\mathrm{Br}(\mathrm{b})$ and $\mathrm{N}(\mathrm{c})$ in the respective SAMs.

\subsection{Characterization of FePt nanoparticle assembly}

Scanning electron microscopy (SEM) micrographs of the nanoparticle monolayer show good coverage all over the substrate surface (Fig. 4.3). SEM analysis (Fig. 4.4) on the linear patterns of the nanoparticles showed $10 \mu \mathrm{m}$ arrays of nanoparticles separated by $5 \mu \mathrm{m}$ distances over the substrate. 


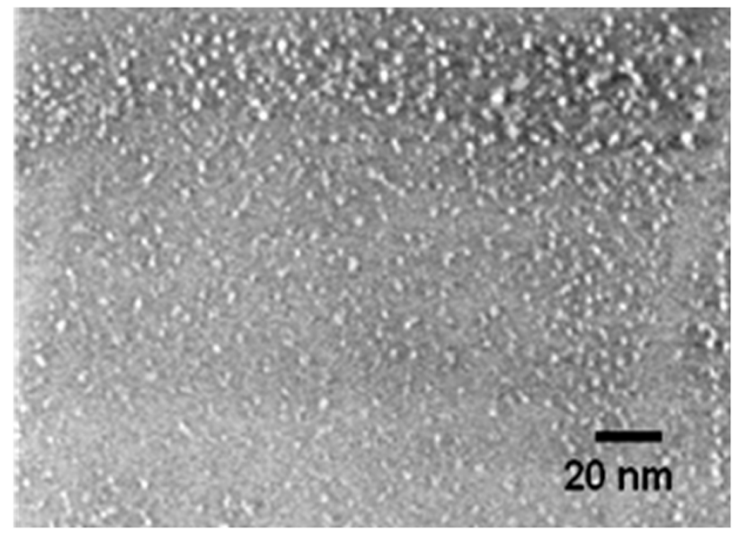

Figure 4.3: SEM micrograph of FePt nanoparticles assembled as a monolayer on the azide terminated silicon oxide substrate.
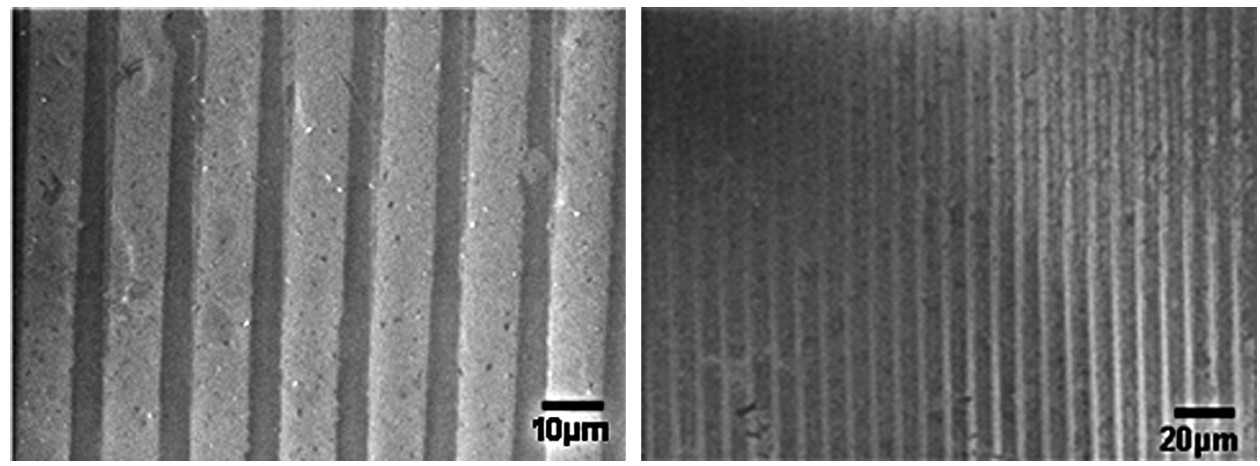

Figure 4.4: SEM micrograph of FePt nanoparticle arrays formed on the silicon oxide substrate by $\mu \mathrm{CP}$. Parallel lines of $10 \mu \mathrm{m}$ are placed $5 \mu \mathrm{m}$ apart.

\subsection{Magnetic properties of FePt nanoparticle assemblies}

Vibrating sample magnetometer (VSM) measurements confirmed magnetic hysteresis for both the full nanoparticle SAMs and the patterned nanoparticle SAMs after annealing, indicating the ferromagnetic character of the particles at room temperature (Fig. 4.5). The ratio of magnetization between full nanoparticle SAMs and the patterned nanoparticle SAMs is about 1.25 , which is slightly lower than the volume ratio of 1.5 . This may due to a small variation in 
substrate size. A larger coercivity is observed in case of a fully covered FePt monolayer compared to the patterned FePt monolayer. This indicates an improved chemical ordering, which translates into enhanced magnetocrystalline anisotropy. The nanoparticle packing density of a fully covered FePt monolayer was found to be higher than the patterned FePt monolayer. This could attribute to the poor nanoparticle inking on the PDMS stamp during the $\mu \mathrm{CP}$ process [32]. The annealing process leads to a higher degree of agglomeration and the agglomerated particles have a larger size and thus a better chemical ordering [11, 33].
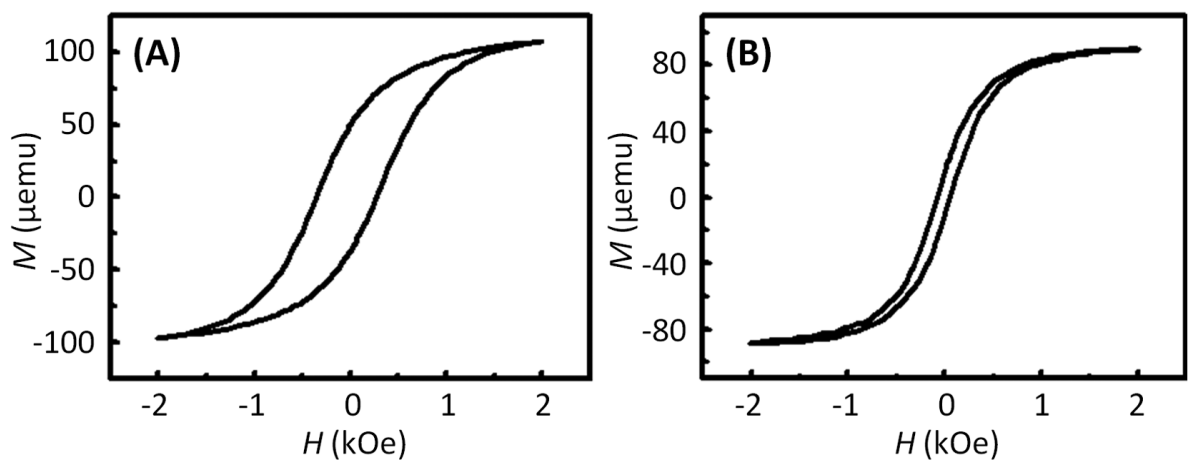

Figure 4.5: VSM magnetization measurements of FePt nanoparticles in a full monolayer (A) and a line-patterned monolayer (B) on the silicon oxide substrate at RT after annealing at $700^{\circ} \mathrm{C}$ under $\mathrm{N}_{2}$ protection. Both the coercive field and the saturation magnetization are reduced in the latter case, see text.

\subsection{Conclusion}

Click-chemistry can be applied to assemble ferromagnetic nanoparticles onto azido-terminated silicon oxide substrates. Monolayers of the nanoparticles were obtained using copper (I) catalyzed click-reaction. Further, we envisage that non-catalyzed click-chemistry between alkyne-functionalized nanoparticles and azide terminated SAMs coupled with $\mu \mathrm{CP}$ can generate well-defined nanoparticles patterns. This methodology has potential for data storage and spintronics applications. 


\subsection{Experimental section}

\section{Synthesis of alkyne-terminated FePt nanoparticles}

Alkyne terminated FePt nanoparticles were synthesized via a modified Sun's method [14]. A solution of $0.25 \mathrm{mmol} \mathrm{Pt}(\mathrm{acac})_{2}$ and $0.75 \mathrm{mmol} 1,2$ hexadecanediol in $20 \mathrm{~mL}$ octyl ether was heated to $80^{\circ} \mathrm{C}$.To this solution 0.5 $\mathrm{mmol}$ oleic acid, $0.5 \mathrm{mmol}$ oleyl amine and $0.5 \mathrm{mmol}$ iron pentacarbonyl were added via a syringe. This mixture was further heated to $150^{\circ} \mathrm{C}$ for $1 \mathrm{~h}$. The black product was precipitated using ethanol, and particles were redispersed in hexane. Replacement of oleic acid and oleyl amine groups by 5-hexanoic acid and 6-amino-1-hexyne was achieved as described by White et al. [28]. First, oleic acid and oleyl amine were stripped off from the nanoparticles by washing with ethanol, collecting the black precipitate, redispersing in hexane (3 times). After washing, the nanoparticles were no longer soluble in hexane, indicating complete removal of the oleic acid and oleyl amine from the nanoparticles surfaces. This was verified by FTIR, which showed complete absence of oleic acid and oleyl amine. Subsequently, the nanoparticles were subjected to ultrasonication for $30 \mathrm{~min}$ with 5-hexanoic acid and 6-amino 1-hexyne (1:1:1 wt\% ratio of FePt: 5-hexanoic acid: 6-amino 1-hexyne) in hexane. Particle sizes were analyzed by TEM (Philips CM-30 Twin operating at $200 \mathrm{kV}$ voltage). A drop of hexane solution of the nanoparticles was deposited on a carbon-coated copper grid. ${ }^{1} \mathrm{H}$ NMR spectra were recorded in d-DMSO on a Varian Unity 300 locked to the deuterated solvent at 300.1 MHz. NMR showed presence of both 5-hexanoic acid and 6-amino 1-hexyne in the appropriate ratio. The nanoparticle sample was analyzed by powder X-ray diffraction (XRD) analysis using a PHILIPS X'Pert diffractometer $\left(\mathrm{CuK}_{\alpha} \lambda=1.5418 \AA\right.$ ).

\section{Preparation of 11-azidoundecyl monolayer on silicon oxide (Scheme 4.1)}

$\mathrm{N}_{3}$ (azide)-terminated monolayers on silicon oxide were obtained according to the methodology previously described [22, 30]. P-doped, (100)-oriented silicon wafers were cut into $10 \times 10 \mathrm{~mm}^{2}$ pieces. First, the silicon wafers were sonicated in ethanol and blow-dried under nitrogen. In order to remove hydrocarbon contaminants and to generate a native oxide layer on the substrate surface, wafers were activated by 15 min exposure to a UV/ozone atmosphere in a plasma 
oxidation setup (Boekel Industries). Subsequently, the substrates were immersed in a $1 \mathrm{mM}$ toluene solution of 11-bromoundecyltrichlorosilane for $45 \mathrm{~min}$ at room temperature. The wafers were rinsed with toluene, acetone, and ethanol to remove any physisorption. This generated a bromo-terminated monolayer on the silicon oxide substrate. In the next step, substitution of the bromo-terminated monolayer with $\mathrm{NaN}_{3}$ led to the formation of azide $\left(\mathrm{N}_{3}\right)$-terminated monolayers on the substrate. This was achieved by immersing the substrates in a saturated sodium azide solution $\left(\mathrm{NaN}_{3}\right)$ in DMF for $48 \mathrm{~h}$ at $70^{\circ} \mathrm{C}$, followed by rinsing with water and ethanol.

Ellipsometric thicknesses were measured with a PLASMOS SD7.01I ellipsometer. Optical constants of $\mathrm{n}=3.865$ and $k=0.019$ for $\mathrm{Si}, n=1.465$ and $k=0$ for $\mathrm{SiO}_{2}$, and $n=1.50$ and $k=0$ for the organic films were used. Raster scans were performed of 25 points per wafer and their values were averaged.

Grazing angle infrared spectra (GAIS) of the SAMs were recorded on a BioRad FTS-60A spectrometer with a liquid nitrogen cooled cryogenic, external mercury cadmium telluride (MCT) detector with its sample area modified to accommodate external reflection sample geometry. The sample area was maintained under nitrogen and spectra were recorded in the mid-IR region (1024 scans) at $4 \mathrm{~cm}^{-1}$ resolution $20 \mathrm{kHz}$ speed.

XPS measurements were performed with Physical Electronics Quantum 2000 equipment, equipped with a spherical sector analyzer and a multichannel plate detector. For the survey scan the pass energy was $117 \mathrm{eV}$, the $\mathrm{X}$-ray beam was set to High Power mode (100 Watt/100 $\mu \mathrm{m})$, and the diameter beam scanned over a $1000 \mu \mathrm{m} \times 500 \mu \mathrm{m}$ area. For element scans the pass energy was $29.35 \mathrm{eV}$, the $X$-ray beam was set to $25 \mathrm{~W} / 100 \mu \mathrm{m}$, and the diameter beam scanned over a $1000 \mu \mathrm{m} \times 500 \mu \mathrm{m}$ area. The excitation source was Al K Alpha monochromatic radiation with a source energy $=1486.6 \mathrm{eV}$. The take-off angle (analyzer angle to sample surface) was set to 30 degrees. The temperature during the analysis was $298 \mathrm{~K}$, and the pressure was between 1 and $3 \times 10^{-8}$ Torr (argon pressure for charge control). For atomic concentration the Shirley background subtraction was employed. The sensitivity factors were provided by Physical Electronics Multipack software version 6.1A. As a reference for surface charging the 
hydrocarbon C1s signal at $284.8 \mathrm{eV}$ was used.

Magnetic studies were carried out using a DMS vibrating sample magnetometer (model VSM10) with fields up to $1500 \mathrm{kA} / \mathrm{m}$ and a sensitivity of $10^{-6} \mathrm{mAm}^{2}$. Measurements were done both on nanoparticles assemblies and patterned nanoparticles on oxidized silicon substrates.

\section{Assembly and pattering of FePt nanoparticles on $\mathrm{SiO}_{2}$ (Scheme 4.1)}

FePt nanoparticles (250 mg) were added to $5 \mathrm{ml}$ of DMSO: $\mathrm{H}_{2} \mathrm{O}(4: 1), \mathrm{CuSO}_{4} 5 \mathrm{H}_{2} \mathrm{O}$ $(62 \mathrm{mg}$ ) and sodium ascorbate $(81 \mathrm{mg}$ ) and deposited on top of the azide modified substrate. Stirring for $48 \mathrm{hrs}$ at RT was followed [30]. The substrate was removed and washed with ethanol to remove the physisorbed layer of nanoparticles. The resulting layer was analyzed with scanning electron microscopy (SEM), performed with a JEOL 5610 apparatus. Samples for transmission electron microscopy (TEM) were prepared by deposition and evaporation of a drop of a solution in chloroform onto amorphous graphite. Linear patterns of nanoparticles were obtained by $\mu \mathrm{CP}$ as described below.

Microcontact printing of nanoparticles: Stamps were fabricated by casting a 10:1 (v/v) mixture of PDMS and curing agent (Sylgard 184, Dow Corning) against a photolithographically patterned silicon master, cured for $1 \mathrm{~h}$ at $60^{\circ} \mathrm{C}$, and released at this curing temperature to avoid build-up of tension due to thermal shrinkage. PDMS stamps, used for $\mu \mathrm{CP}$, were left in the oven at $60^{\circ} \mathrm{C}$ for at least $18 \mathrm{~h}$ to ensure complete curing. Subsequently, the stamps were inked by immersion into the adsorbate solution of nanoparticles (DMSO: $\mathrm{H}_{2} \mathrm{O}=4: 1 \mathrm{v} / \mathrm{v}$ ) for $15 \mathrm{~min}$. After withdrawal from the solution and drying under a continuous stream of nitrogen for $1 \mathrm{~min}$, the stamps were applied with conformal contact for 5 min on the azide SAMs. Re-inking was done after each printing step. This process was carried out without using any $\mathrm{Cu}(\mathrm{I})$ catalyst. Finally, the substrates were systematically rinsed with water and ethanol. 


\section{References}

[1] T. Hyeon, Chemical Communications (2003) 927.

[2] U. Jeong, X. W. Teng, Y. Wang, H. Yang, Y. N. Xia, Advanced Materials 19 (2007) 33.

[3] P. Tartaj, M. P. Morales, T. Gonzalez-Carreno, S. Veintemillas-Verdaguer, C. J. Serna, Journal of Magnetism and Magnetic Materials 290 (2005) 28.

[4] G. C. Hadjipanayis, M. Marinescu, Y. H. Huang, M. J. Bonder, A. Gabay, Sensor Letters 5 (2007) 1.

[5] W. J. M. Naber, S. Faez, W. G. van der Wiel, Journal of Physics D-Applied Physics 40 (2007) R205.

[6] A. Hernando, P. Crespo, M. A. Garcia, Thescientificworldjournal 5 (2005) 972.

[7] S. S. Kang, G. X. Miao, S. Shi, Z. Jia, D. E. Nikles, J. W. Harrell, Journal of the American Chemical Society 128 (2006) 1042.

[8] S. S. Kang, S. Shi, G. X. Miao, Z. Y. Jia, D. E. Nikles, J. W. Harrell, Journal of Nanoscience and Nanotechnology 7 (2007) 350.

[9] B. Rellinghaus, S. Stappert, M. Acet, E. F. Wassermann, Journal of Magnetism and Magnetic Materials 266 (2003) 142.

[10] B. D. Terris, T. Thomson, Journal of Physics D-Applied Physics 38 (2005) R199.

[11] T. Schrefl, G. Hrkac, D. Suess, W. Scholz, J. Fidler, Journal of Applied Physics 93 (2003) 7041.

[12] M. Acet, C. Mayer, O. Muth, A. Terheiden, G. Dyker, Journal of Crystal Growth 285 (2005) 365.

[13] S. H. Sun, S. Anders, H. F. Hamann, J. U. Thiele, J. E. E. Baglin, T. Thomson, E. E. Fullerton, C. B. Murray, B. D. Terris, Journal of the American Chemical Society 124 (2002) 2884.

[14] S. H. Sun, S. Anders, T. Thomson, J. E. E. Baglin, M. F. Toney, H. F. Hamann, C. B. Murray, B. D. Terris, Journal of Physical Chemistry B 107 (2003) 5419.

[15]A. C. C. Yu, M. Mizuno, Y. Sasaki, M. Inoue, H. Kondo, I. Ohta, D. Djayaprawira, 
M. Takahashi, Applied Physics Letters 82 (2003) 4352.

[16] H. Nandivada, X. W. Jiang, J. Lahann, Advanced Materials 19 (2007) 2197.

[17] W. H. Binder, C. Kluger, Current Organic Chemistry 10 (2006) 1791.

[18] W. H. Binder, R. Sachsenhofer, C. J. Straif, R. Zirbs, Journal of Materials Chemistry 17 (2007) 2125.

[19] J. L. Brennan, N. S. Hatzakis, T. R. Tshikhudo, N. Dirvianskyte, V. Razumas, S. Patkar, J. Vind, A. Svendsen, R. J. M. Nolte, A. E. Rowan, M. Brust, Bioconjugate Chemistry 17 (2006) 1373.

[20] D. Fournier, R. Hoogenboom, U. S. Schubert, Chemical Society Reviews 36 (2007) 1369.

[21] M. V. Gil, M. J. Arevalo, O. Lopez, Synthesis-Stuttgart (2007) 1589.

[22] T. Lummerstorfer, H. Hoffmann, Journal of Physical Chemistry B 108 (2004) 3963.

[23] J. E. Moses, A. D. Moorhouse, Chemical Society Reviews 36 (2007) 1249.

[24] S. Narayan, J. Muldoon, M. G. Finn, V. V. Fokin, H. C. Kolb, K. B. Sharpless, Angewandte Chemie-International Edition 44 (2005) 3275.

[25] V. O. Rodionov, V. V. Fokin, M. G. Finn, Angewandte Chemie-International Edition 44 (2005) 2210.

[26] C. J. Hawker, K. L. Wooley, Science 309 (2005) 1200.

[27] D. A. Fleming, C. J. Thode, M. E. Williams, Chemistry of Materials 18 (2006) 2327.

[28] M. A. White, J. A. Johnson, J. T. Koberstein, N. J. Turro, Journal of the American Chemical Society 128 (2006) 11356.

[29] P. C. Lin, S. H. Ueng, S. C. Yu, M. D. Jan, A. K. Adak, C. C. Yu, C. C. Lin, Organic Letters 9 (2007) 2131.

[30] W. H. Binder, M. Lomoschitz, R. Sachsenhofer, G. Friedbacher, Journal of Nanomaterials (2009) 
[31] D. I. Rozkiewicz, D. Janczewski, W. Verboom, B. J. Ravoo, D. N. Reinhoudt, Angewandte Chemie-International Edition 45 (2006) 5292.

[32] H. Xu, A. Gomez-Casado, Z. Liu, D. N. Reinhoudt, R. G. H. Lammertink, J. Huskens, Langmuir 25 (2009) 13972.

[33] H. M. Lu, Z. H. Cao, C. L. Zhao, P. Y. Li, X. K. Meng, Journal of Applied Physics 103 (2008) 4. 


\section{Nano-patterned monolayer and multilayer of FePtAu nanoparticles on aluminum oxide substrate}

Owing to the superior dielectric property of aluminum oxide, precise patterning of self-assembled monolayers (SAMs) and nanoparticles (NPS) on aluminum oxide substrates is highly interesting for generating SAM- or NP-based electronic devices. This study employed nanoimprint lithography (NIL) and nanomolding in capillaries (NAMIC) for patterning ferromagnetic NPs on aluminum oxide substrates. The fabrication of structured arrays of various SAMs and NPs in micrometer and nanometer ranges was demonstrated. The polymer template generated by NIL behaved as a physical barrier and defined the pattern areas on the substrate. FePtAu NPs were assembled on phosph(on)ate SAM-modified polymer patterned substrates. After polymer removal, nano- and microscale, line and dot NP patterns, with controlled layer thickness, were obtained on aluminum oxide substrates. Thick nanolines of NPs were obtained by NAMIC. $\mathrm{PO}_{3}$-terminated FePtAu NPs were assembled on alumina without need of a linker. The magnetic properties of the NPs were addressed by vibrating sample magnetometry and those of the patterned NPs by magnetic force microscopy.

This chapter has been published as T. Gang, O. Yildirim, S. Kinge, X. Duan, D.N. Reinhoudt, D.H.A. Blank, G. Rijnders, W.G. van der Wiel, and J. Huskens, Journal of Materials Chemistry, article in press. 


\subsection{Introduction}

Since the discovery of giant magnetoresistance and tunnel magnetoresistance in thin-film structures of alternating magnetic and non-magnetic layers, the field of spintronics has acquired massive scientific and industrial attention [1]. Driven by the need for higher integration density in magnetic and spintronic devices, spin-dependent transport in magnetic nanostructures has been extensively studied [2-3]. Most of the miniaturized spintronic devices were fabricated via a top-down approach involving metal/ oxide deposition, lithography and etching etc. Magnetic NP assembly potentially offers an alternative, cost-effective bottom-up approach to build nanoscale spintronic devices [4-7]. For instance, monolayers of magnetic nanoparticles can potentially replace the magnetic layers in conventional magnetic tunnel junctions. Moreover, magnetic nanoparticles may lead to ultra-small magnetic devices (ultimately down to the size of a single nanoparticle), thus offering high integration density [8-9]. To achieve this, it is essential to have a well-controlled patterning and assembly method to position the magnetic NPs on metal-oxide substrates, such as aluminum oxide which is widely used as the dielectric material in spintronics devices [4, 10-12].

Several attempts were reported to address this issue, however those methods have their limitations. For instance, photo-patterned SAMs on metal oxides have been used to attach nanoparticles[13-14] and a contact printing method was demonstrated to create $\mathrm{Pt} @ \mathrm{Fe}_{2} \mathrm{O}_{3}$ core-shell nanoparticle patterns on silicon substrates [15]. In these cases, the dimension, thickness and shape of the patterns are limited by the constraints of the fabrication process.

In this chapter, a robust approach to form patterned magnetic nanoparticle assemblies with high resolution and high selectivity on aluminum oxide substrates is demonstrated. Ferromagnetic NP patterns of micrometer and nanometer scale on aluminum oxide sbstrate were prepared by combining NIL or NAMIC with SAMs. FePtAu and FePt NPs were used because they have high chemical stability, strong magnetocrystalline anisotropy and very small size distribution [16-17]. Various $\mathrm{PO}_{3}$-terminated molecular linkers were used to anchor the NPs onto alumina. Ferromagnetic properties of the NPs and NP 
patterns were addressed with a vibrating sample magnetometer (VSM) and MFM, respectively.

\section{2 $\mathrm{PO}_{3}$-terminated FePtAu nanoparticles $\left(\mathrm{PO}_{3}-\mathrm{NPs}\right)$}

The methyl-terminated FePtAu NPs and FePt NPs were prepared as described in Chapter 3. These FePtAu or FePt NPs were $\cong 4 \mathrm{~nm}$ in diameter and had a coercivity of $10 \mathrm{kOe}$ after annealing under reducing environment $\left(96 \% \mathrm{Ar} / 4 \% \mathrm{H}_{2}\right)$. The $\mathrm{PO}_{3}-N P s$ were prepared from methyl-terminated FePtAu NPs (Fig. 5.1a) through ligand exchange with Mercaptoundecylphosphonic acid (MUP) (Fig. 5.1b) using a modification of the procedure reported by Bagaria et al. [18]. The particles were dispersed in ethanol:Et3 $\mathrm{N}(10: 1)$.

(a)

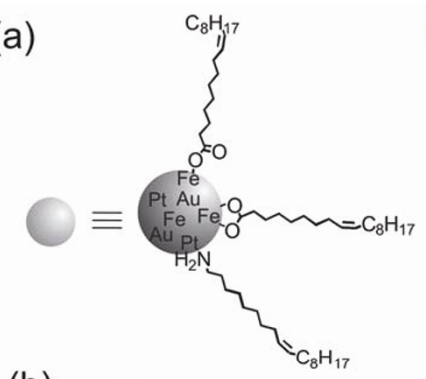

(b)

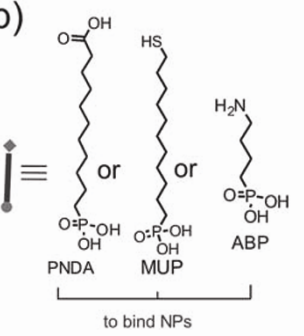

(c)
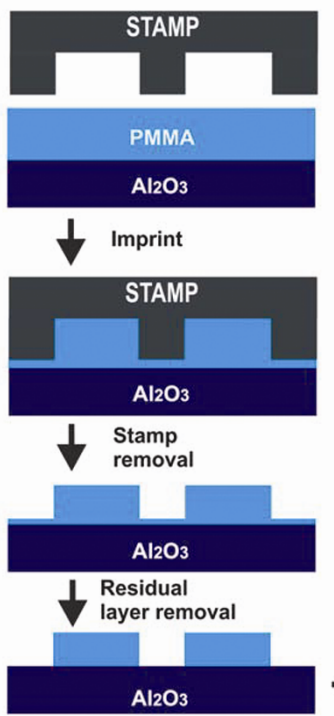

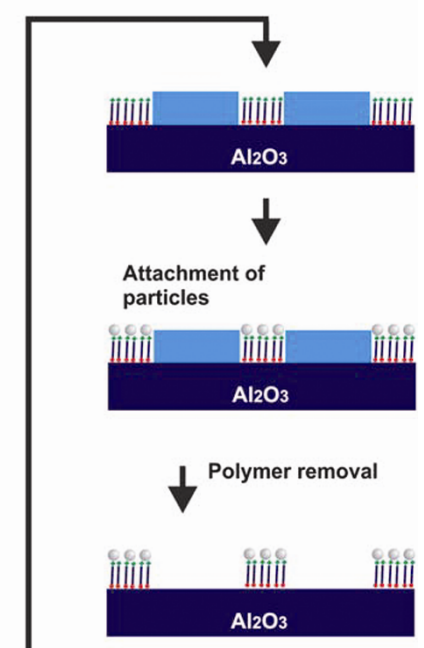

$\mathrm{Al}_{2} \mathrm{O}_{3}$

Figure 5.1: (a) FePtAu NPs stabilized with oleic acid and oleyl amine. (b) The organic molecules used to attach the NPs to the alumina substrate. (c) Patterns of NPs are formed by attachment through ligand exchange onto amino (aminobutylphosphonic acid, ABP), carboxylic acid (phosphonoundecanoic acid, PUD), and thiol (mercaptoundecylphosphonic acid, MUP) functionalized monolayer-modified alumina substrates. 


\subsection{SAM patterns prepared by NIL}

Tetradecylphosphate (TDP) SAM lines were prepared as shown in Fig. 5.1. The residual layer was removed by oxygen plasma, which enabled anisotropic etching and positive transfer of the mold features onto the substrate. A uniform TDP pattern was formed with homogeneous thickness, corresponding to one SAM layer (Fig. 5.2a). Nanopatterns of TDP SAMs were also prepared with a similar method, but it was not possible to get a clear image probably due to the quite small size of the patterns. Alumina appears bright in the friction image (Fig. 5.2) because of a higher friction force relative to the TDP area. The TDP pattern was back-filled with $\mathrm{N}$-[3- (trimethoxysilyl)propyl)ethylene]-diamine from the gas phase to chemically pattern the substrate with two different kinds of SAMs. The friction force of the more hydrophilic $\mathrm{NH}_{2}$-terminated area is higher compared to the $\mathrm{CH}_{3}$-terminated TDP region (Fig. 5.2c).

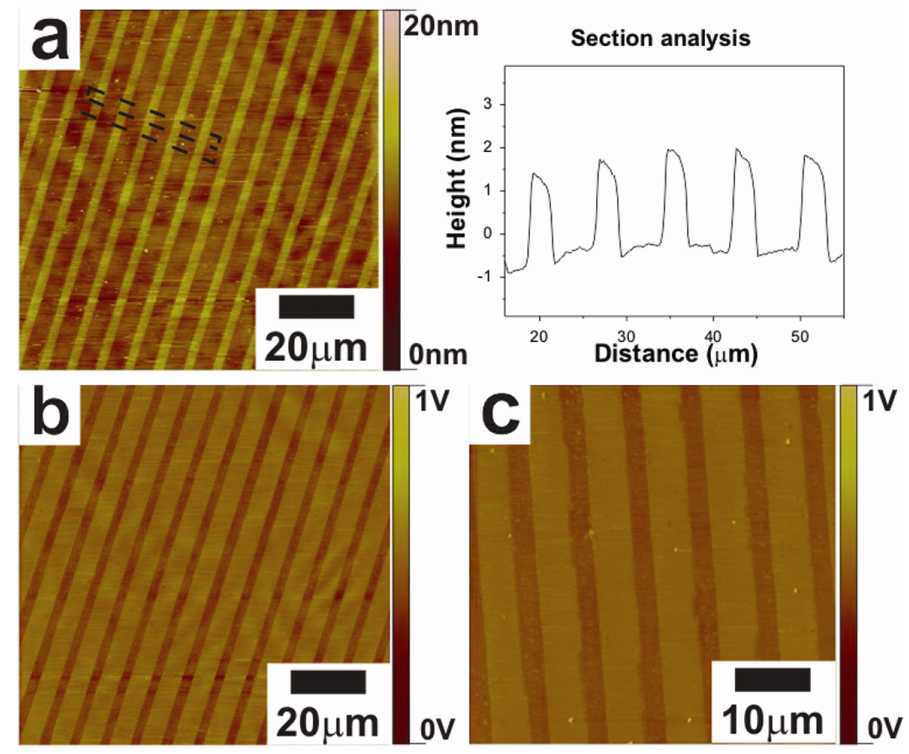

Figure 5.2: Contact-mode height (a) and friction (b) AFM images of $5 \mu \mathrm{m}$ lines of tetradecylphosphate (TDP) on $\mathrm{Al}_{2} \mathrm{O}_{3}$ with $8 \mu \mathrm{m}$ period; (c) friction AFM image after subsequent evaporation of an amino alkyl silane. In the friction images, brighter areas indicate higher friction. 


\subsection{Nanoparticle pattern preparation by NIL and SAMs}

Figure 5.3 shows the morphologies of the FePtAu patterns on $\mathrm{Al}_{2} \mathrm{O}_{3}$ substrates prepared by NIL and self-assembly as described in Fig. 5.1. PMMA was spin-coated on $\mathrm{Al}_{2} \mathrm{O}_{3}$ substrates with $460 \mathrm{~nm}$ and $120 \mathrm{~nm}$ thickness for micron and nanosize patterning, respectively. NIL was performed at a pressure of $40 \mathrm{bar}$ at $180{ }^{\circ} \mathrm{C}$. The residual layer was removed by oxygen plasma etching. The nanoparticles were assembled on $\mathrm{NH}_{2-}$, $\mathrm{SH}$ - and $\mathrm{COOH}$-terminated SAM patterns. Figures 5.3a and $b$ show that lines of FePtAu below $200 \mathrm{~nm}$ width formed by using a nano-mold and PUD as the linker. The heights of the FePtAu NP patterns were around $6 \mathrm{~nm}$, which demonstrates good monolayer coverage. For the micron patterns, a similar height of FePtAu NP features was observed (not shown). An important advantage of nanoimprint lithography is the good edge definition. When compared to NP patterns prepared by using microcontact printing [4], NIL patterns have well-defined edges, and better contrast between patterned and bare regions. The FePtAu NP densities, calculated from AFM images, were similar for MUP and PUD-patterned surfaces, $(1.6 \pm 0.1) \times 10^{10}$ and $(1.5 \pm 0.1) \times 10^{10} \mathrm{NPs} / \mathrm{cm}^{2}$, respectively. Since a similar coverage was reached with immersion time of $10 \mathrm{~min}$ for $-\mathrm{SH}$ and $-\mathrm{COOH}$-modified surfaces, the relative rates of binding of NPs on modified surfaces are similar for MUP and PUD.

The shape of the patterns is defined by the shape of the mold. As seen in Fig. $5.3 \mathrm{~d}$, dot patterns can also be prepared as well as lines. The lateral dimensions of the patterns were in good agreement with the feature size of the molds. The process is not limited to FePt NPs, any particle or molecule which has affinity to the end group of the SAM layer can bind to the surface to form a pattern. 

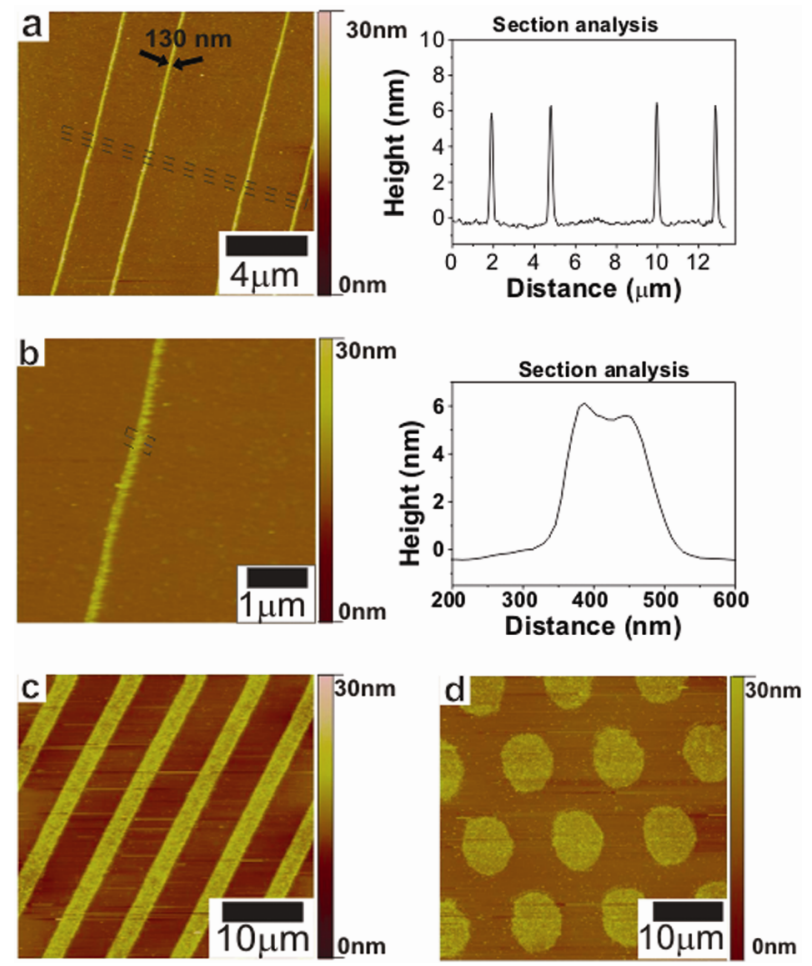

Figure 5.3: Tapping-mode (TM) AFM height images of nanoparticles adsorbed on SAM-modified $\mathrm{Al}_{2} \mathrm{O}_{3}$ substrates. FePtAu nanoparticles, (a, b) $130 \mathrm{~nm}$ wide PUD lines, 10 min immersion, (c) $3 \mu \mathrm{m}$ PUD lines, 10 min immersion, (d) $10 \mu \mathrm{m}$ MUP dots, $10 \mathrm{~min}$ immersion.

\subsection{Magnetic properties of multilayer of FePt NPs prepared by NIL}

The magnetic properties of patterned FePt NPs were studied with MFM instead of VSM, since the substrate size for pattering studies were larger than the maximal sample size of the VSM $(5 \times 5 \mathrm{~mm})$. MFM is known to be a powerful technique for studying micro-/nanopatterned magnetic structures [15]. It can effectively separate the magnetic interaction from the topographical information of the sample. Due to the magnetic interaction between the magnetic dipoles of the sample and the oscillating tip, a phase contrast is observed which gives information on the magnetic state independent of the topography [15]. The phase contrast can be either positive or negative, which corresponds to attractive 
or repulsive forces between the tip and the sample. Thick FePt NP patterns were prepared by using a concentrated solution of NPs. A drop of a $20 \mathrm{mg} / \mathrm{ml}$ solution of FePt NPs in hexane was deposited on a PMMA-PUD patterned alumina sample. The NPs precipitated on the surface upon evaporation of hexane and formed a multilayer due to the high concentration. Then the sample was gently immersed in acetone to remove PMMA without damaging the NP layer. The sample was further annealed to transfer the NPs into the ferromagnetic L1o phase [4]. Instead of a monolayer, a thick layer was used to have a strong enough magnetic signal for MFM measurements. Figure 5.4 shows the AFM and corresponding MFM image of the FePt lines after annealing, which were recorded simultaneously. The height of the patterns was about $35 \mathrm{~nm}$ which corresponds to 8-9 layers of NPs and the width was around $5 \mu \mathrm{m}$ (Fig. 5.4a). The magnetic contrast in the MFM phase image is clearly visible and as expected (Fig. 5.4b), is not identical to the AFM height image (Fig. 5.4a). This is due to the random momentum directions of the NPs giving a non-homogeneous magnetic contrast. In other words, some NPs attract the magnetic tip while others repel it. Magnetization direction of the tip is perpendicular to the sample surface. In some regions the magnetic contrast is weaker than at other places, which may be due to the small particle size, differences in coverage, or to the momentum direction of NPs being perpendicular to the magnetization of the tip.
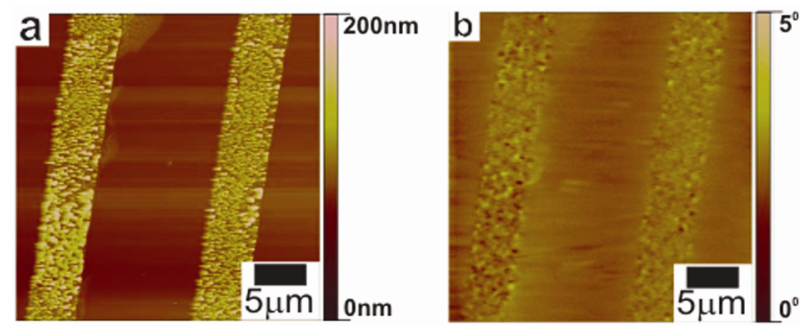

Figure 5.4: AFM height (a) and MFM phase (b) images of FePt NP multilayer patterns after annealing under reducing environment $\left(96 \% \mathrm{Ar} / 4 \% \mathrm{H}_{2}\right)$ for $2 \mathrm{~h}$ at $800{ }^{\circ} \mathrm{C}$.

\subsection{Assembly of $\mathrm{PO}_{3}$-terminated FePtAu NPs}

MUP-stabilized $\mathrm{PO}_{3}-\mathrm{NPs}$ (Fig. 5.5a) allow direct assembly of NPs onto an $\mathrm{Al}_{2} \mathrm{O}_{3}$ surface without the need for a linker SAM on the substrate. Figure 5.5 shows the 
morphology of the alumina sample after immersion in the $\mathrm{PO}_{3}-\mathrm{NP}$ solution for $180 \mathrm{~min}$. The NP density is $(4.2 \pm 0.2) \times 10^{10} \mathrm{NPs} / \mathrm{cm}^{2}$. This is 5 times higher than the NP density for FePt on bare $\mathrm{Al}_{2} \mathrm{O}_{3}$ after 90 min immersion [4], which shows that the $\mathrm{PO}_{3}-\mathrm{NPs}$ are probably attached to the surface through the $\mathrm{PO}_{3}$ groups and that the high density is not due to non-specific adsorption. This kind of particles may be assembled on pre-patterned surfaces protected by methyl terminated regions to create NP patterns.
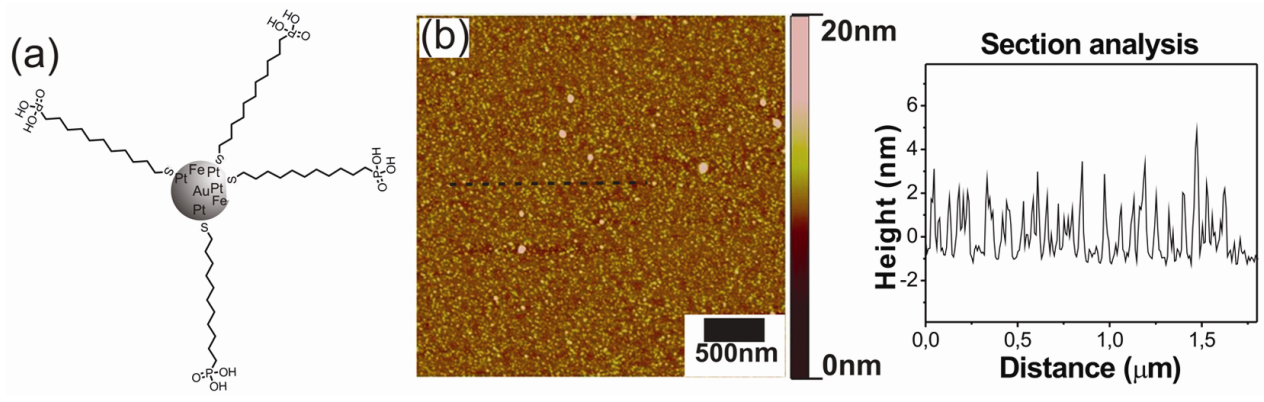

Figure 5.5: (a) MUP-stabilized $\mathrm{PO}_{3}$-terminated FePtAu NPs $\left(\mathrm{PO}_{3}-\mathrm{NP}\right)$ (b) AFM image and section analysis of the $\mathrm{PO}_{3}$-NPs assembled on a bare $\mathrm{Al}_{2} \mathrm{O}_{3}$ substrate after 180 min immersion.

\subsection{Multilayers of $\mathrm{PO}_{3}$-terminated FePtAu NP patterns prepared by NAMIC}

$\mathrm{PO}_{3}$-NPs were used to create NP patterns on PUD-modified alumina, by NAMIC, as illustrated in Fig. 5.6a. The main reason to use $\mathrm{PO}_{3}-\mathrm{NPs}$ dispersed in ethanol + $\mathrm{Et}_{3} \mathrm{~N}$ is to prevent PDMS from swelling. Hexane was not used as a solvent, because it would destroy uniform contact and cause pattern deformation upon swelling. As seen in Fig. 5.6b and c continuous nanolines of FePtAu NPs were prepared without obvious defects over a large area. The shape of the pattern is in good agreement with the stamp used. The width of the NP patterns is close to the width of the nano-channels (Fig. 5.6e). Multilayers were achieved probably due to irreversible aggregation during drying. The height of the NP patterns was around $70 \mathrm{~nm}$, which corresponds to 15-20 layers of NPs. This value is lower than the nano-channel height $(100 \mathrm{~nm})$. This may be due to shrinkage after drying, to 
a low concentration of particles in the NP dispersion, or to the use of oxygen plasma on the mold before NAMIC. NAMIC enables to prepare densely packed NP multilayers, the height can be controlled by the height of the channels.
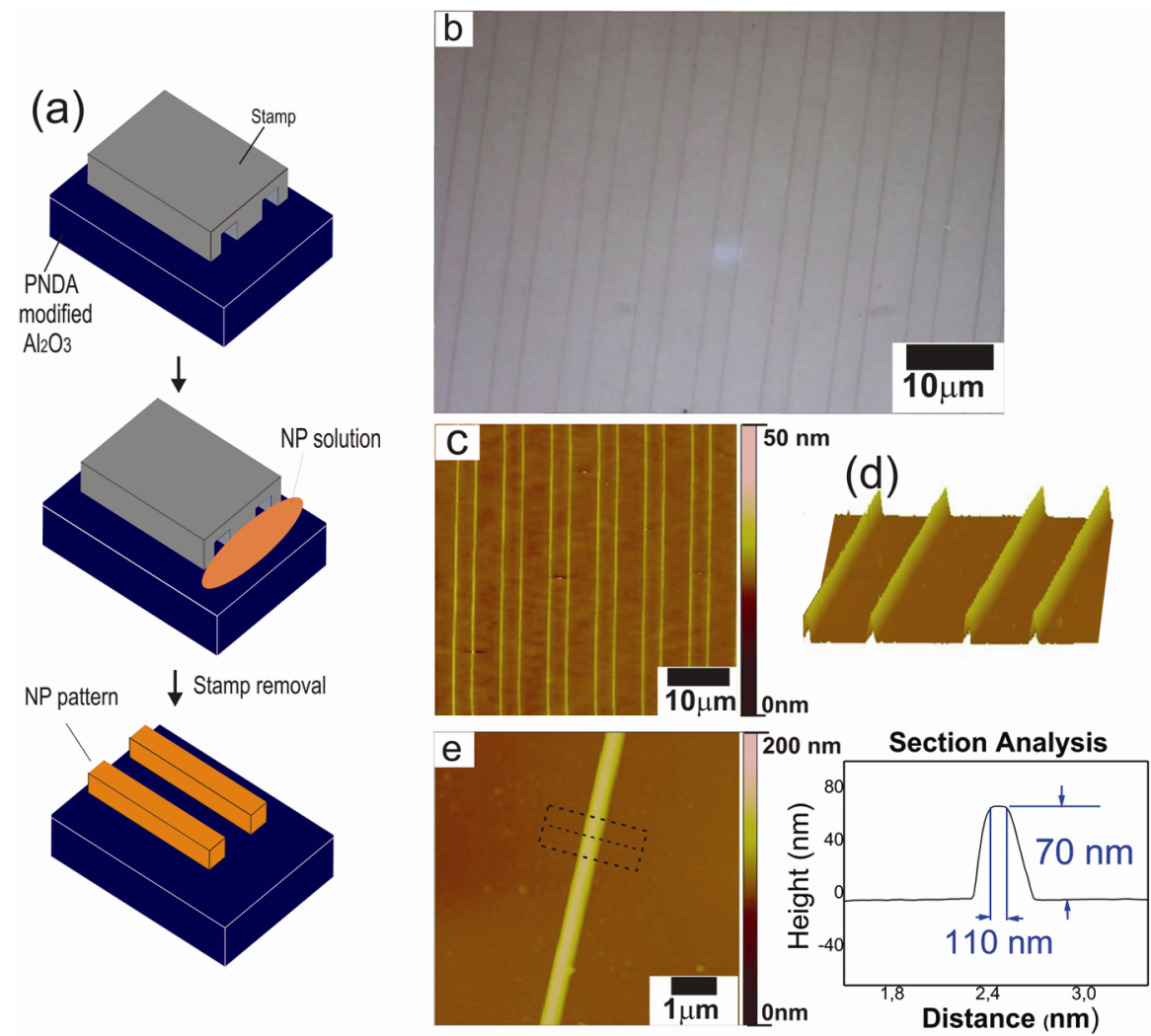

Figure 5.6: (a) The NAMIC process. FePtAu NP patterns prepared by NAMIC on a PUD-modified alumina substrate, (b) optical image, (c,e) AFM height image with section analysis, (d) 3D AFM topography image.

\subsection{Conclusion}

By combining NIL or NAMIC with SAMs of phosph(on)ate molecular linkers, FePtAu NP mono/multilayer patterns with high resolution below $150 \mathrm{~nm}$ as well as in micron range were demonstrated. High selectivity between the patterned and non-patterned regions was achieved. The ability to pattern magnetic nanoparticles at the nanoscale with high selectivity on metal oxide substrates 
can be considered as an important step towards the realization of ultra-small and cost-effective magnetic nanoparticle based spintronic devices.

\subsection{Experimental section}

Polished substrates of $\mathrm{R}-(102) \mathrm{Al}_{2} \mathrm{O}_{3}\left(1 \times 10 \times 10 \mathrm{~mm}^{3}\right)$ were purchased from SurfaceNet $\mathrm{GmbH}$, Germany. These substrates were cleaned by ultrasonication in acetone and ethanol for $30 \mathrm{~min}$ each. Tetradecylphosphoric acid (TDP) was supplied by A. Wagenaar and J. Engbersen (RUG, Groningen). Phosphonoundecanoic acid (PUD, purity 96\%), PMMA (weight-average molecular weight, $M_{w} 350$ kD), N-[3-(trimethoxysilyl)propyl)ethylene]-diamine, Pt(acac)2, oleic acid and silver enhancer solutions $a$ and $b$ were purchased from Sigma-Aldrich. Oleyl amine was purchased from Fluka. Hexadecanediol and iron pentacarbonyl were purchased from ABCR. Mercaptoundecylphosphonic acid (MUP) was synthesized according to a literature method [8].

Synthesis of $\mathrm{PO}_{3}$ terminated FePtAu NPs: The NPs were prepared using a modification of the procedure reported by Bagaria et al. [3a, 10] Cyclohexanone was chosen as the solvent for the ligand exchange because it dissolves FePt NPs and mercaptoundecylphosphonic acid (MUP). A solution of MUP was prepared by mixing $2.7 \mathrm{mg}$ MUP with $5 \mathrm{ml}$ cyclohexanone. $50 \mathrm{mg}$ of FePtAu nanoparticles dispersed in $0.5 \mathrm{ml}$ hexane was added to this solution. The mixture stired overnight, then centrifuged and the supernatant was discarded. Cyclohexanone $(10 \mathrm{ml})$ was added to the precipitate to remove FePtAu with oleic acid and oleylamine ligands and any excess MUP. The solution was centrifuged and the supernatant was discarded. As a final cleaning, the precipitate was mixed with 10 $\mathrm{ml}$ acetone. The solution was again centrifuged and the supernatant was discarded. The particles were then dispersed in $3 \mathrm{ml}$ of ethanol:Et $3 \mathrm{~N}$ (10:1).

Nanoimprint lithography (NIL): The molds were fabricated by photolithography followed by reactive-ion etching (RIE, Elektrotech Twin system PF 340) or by EBL followed by titanium evaporation and lift-off. They consisted of $5 \mu \mathrm{m}$ wide lines at $15 \mu \mathrm{m}$ period, $3 \mu \mathrm{m}$ lines with $8 \mu \mathrm{m}$ period with a height of $570 \mathrm{~nm}$ or $100 \mathrm{~nm}$ lines with around $4 \mu \mathrm{m}$ period with a height of $125 \mathrm{~nm}$. $1 H, 1 H, 2 H$, 
$2 \mathrm{H}$-perfluorodecyltrichlorosilane was used as an anti-adherent layer to facilitate the stamp-imprint separation. $\mathrm{Al}_{2} \mathrm{O}_{3}$ substrates were cleaned by oxygen-plasma for 7 minutes and covered with a $460 \mathrm{~nm}$ thick layer of PMMA by spin-coating in case of micron size patterning. Stamp and substrate were put in contact and pressure of 40 bar was appliedat $180{ }^{\circ} \mathrm{C}$ using a hydraulic press (Specac). The residual layer was removed by dipping the samples in acetone for $25 \mathrm{sec}$ or by oxygen-plasma for $90 \mathrm{~s}$ using a RIE system. For nano patterns $120 \mathrm{~nm}$ thick PMMA and $18 \mathrm{~s}$ RIE was done. In the last step of NP pattern fabrication, the polymer layer was removed by 5 minutes acetone followed by 5 minutes chloroform immersion. For TDP SAM patterns, polymer layer was removed by 3 hours ultrasonication in acetone. The imprint cycle was $30 \mathrm{~min}$.

Monolayer patterning using NIL: PMMA paterned $\mathrm{Al}_{2} \mathrm{O}_{3}$ substrates were immersed into $0.125 \mathrm{mM}$ TDP solution in 100:1 v/v hexane:isopropanol, a $1 \mathrm{mM}$ PUD solution in 50:50 v/v ethanol: $\mathrm{H}_{2} \mathrm{O}$ or a $0.5 \mathrm{mM}$ mM MUP solution in ethanol for 2 days at room temperature. Afterwards, the samples were rinsed with the corresponding pure solvents or solvent mixtures, and dried under a flow of $\mathrm{N}_{2}$.

Nanoparticle assembly: $\mathrm{Al}_{2} \mathrm{O}_{3}$ substrates covered with PMMA and self-assembled monolayer (SAM) patterns of PUD or MUP were immersed into a FePtAu $(0.250 \mathrm{mg} / \mathrm{ml}) \mathrm{NP}$ solution for $10-95 \mathrm{~min}$ to assemble NPs on the modified $\mathrm{Al}_{2} \mathrm{O}_{3}$ surfaces. For VSM measurement, fully MUP-covered $\mathrm{Al}_{2} \mathrm{O}_{3}$ substrate was immersed into a FePtAu $(0.250 \mathrm{mg} / \mathrm{ml})$ solution for $10 \mathrm{~min}$. Subsequently the samples were rinsed with pure hexane to wash off physisorbed particles. After PMMA removal by actione, patterns of NP monolayer was formed.

FePt NP multilayer patterns were prepared by using a concentrated solution of NPs. A drop of a $20 \mathrm{mg} / \mathrm{ml}$ solution of FePt NPs in hexane was deposited on the PMMA-PUD SAM patterned alumina substrate. The sample was dried for $30 \mathrm{~min}$ under ambient conditons in a fume hood. The NPs precipitated on the surface upon evaporation of hexane and formed a multilayer due to the high concentration. Then the sample was gently immersed in acetone to remove PMMA without damaging the NP multilayer patterns.

Nanomolding in capillaries (NAMIC): Before NAMIC, the PDMS nanomold was 
shortly treated with oxygen plasma to promote adhesion upon contacting the substrate [11]. $100 \mathrm{~nm}$ line features with $100 \mathrm{~nm}$ height was brought into conformal contact with PUD-functionalized alumina substrates. A drop of $\mathrm{PO}_{3}$-terminated FePtAu NPs functionalized with MUP and dispersed in ethanol:Et $3 \mathrm{~N}$ was put at one part of the PDMS for filling in the channels through capillary force. Here, FePtAu dispersed in hexane was not used since hexane causes swelling of PDMS which would cause non-uniform contact between the stamp and the substrate. The sample was left for solvent evaporation and NP precipitation in the nano-channels. After $10 \mathrm{hr}$ the PDMS was removed, the residual polymeric material was rinsed with xylene followed by oxygen plasma and the substrate with line patterns of FePtAu was imaged by AFM and optical microscopy.

\section{Measurements}

Atomic force microscopy (AFM): The morphology of the nanoparticle-covered surfaces was observed by a digital multimode Nanoscope III (Digital Instruments, Santa Barbara, CA) scanning force microscope, equipped with a J-scanner. All measurements were done at ambient in tapping mode, contact mode. The magnetic force microscopy (MFM) was done by a Dimension 3100 (Digital Instruments, Santa Barbara, CA). The MFM tips are provided by SmartTip (type SC-20-M). The approximate nanoparticle densities were calculated by counting particles in a certain area. A standard deviation of $5 \%$ was assumed based on our previous study [4].

Vibrating Sample Magnetometry (VSM): Magnetic studies were carried out using a DMS Vibrating Sample Magnetometer (model VSM10) with fields up to $1500 \mathrm{kA} / \mathrm{m}$ and a sensitivity of $10^{-6} \mathrm{mAm}^{2}$. Measurements were done on NP assembly on MUP-modified $\mathrm{Al}_{2} \mathrm{O}_{3}$ substrate

X-Ray Diffractometry (XRD): The nanoparticle samples after annealing were analyzed by powder X-ray diffraction (XRD) analysis using a Philips X'Pert diffractometer $\left(\mathrm{CuK}_{\alpha} \lambda=1.5418 \AA\right.$ ) $)$.

High-Resolution Transmission Electron Microscopy (HRTEM-EDX): Particle sizes were analyzed by TEM (Philips CM-30 Twin operating at $200 \mathrm{kV}$ voltage). A drop of hexane solution of the NPs was deposited on a carbon-coated copper grid. 


\section{References}

[1] J. G. J. Zhu, C. D. Park, Materials Today 9 (2006) 36.

[2] Y. Okutomi, K. Miyake, M. Doi, H. N. Fuke, H. Iwasaki, M. Sahashi, Journal of Applied Physics 109 (2011)

[3] H. Tomita, T. Nozaki, T. Seki, T. Nagase, K. Nishiyama, E. Kitagawa, M. Yoshikawa, T. Daibou, M. Nagamine, T. Kishi, S. Ikegawa, N. Shimomura, H. Yoda, Y. Suzuki, IEEE Transactions on Magnetics 47 (2011) 1599.

[4] O. Yildirim, T. Gang, S. Kinge, D. N. Reinhoudt, D. H. A. Blank, W. G. van der Wiel, G. Rijnders, J. Huskens, International Journal of Molecular Sciences 11 (2010) 1162.

[5] S. Kinge, T. Gang, W. J. M. Naber, W. G. van der Wiel, D. N. Reinhoudt, Langmuir 27 (2010) 570.

[6] S. Kinge, T. Gang, W. J. M. Naber, H. Boschker, G. Rijnders, D. N. Reinhoudt, W. G. van der Wiel, Nano Letters 9 (2009) 3220.

[7] W. J. M. Naber, S. Faez, W. G. van der Wiel, Journal of Physics D-Applied Physics 40 (2007) R205.

[8] C. T. Black, C. B. Murray, R. L. Sandstrom, S. H. Sun, Science 290 (2000) 1131.

[9] K. Liu, J. Nogues, C. Leighton, H. Masuda, K. Nishio, I. V. Roshchin, I. K. Schuller, Applied Physics Letters 81 (2002) 4434.

[10] S. Yuasa, T. Nagahama, Y. Suzuki, Science 297 (2002) 234.

[11] D. X. Wang, C. Nordman, J. M. Daughton, Z. H. Qian, J. Fink, IEEE Transactions on Magnetics 40 (2004) 2269.

[12] J. Schmalhorst, G. Reiss, Journal of Magnetism and Magnetic Materials 272-276 (2004) E1485.

[13] H. Sugimura, A. Hozumi, T. Kameyama, O. Takai, Advanced Materials 13 (2001) 667.

[14] S. Q. Sun, G. J. Leggett, Nano Letters 7 (2007) 3753.

[15] Q. J. Guo, X. W. Teng, H. Yang, Nano Letters 4 (2004) 1657. 
[16] S. Kinge, T. Gang, W. J. M. Naber, H. Boschker, G. Rijnders, D. N. Reinhoudt, W. G. van der Wiel, Nano Letters 9 (2009) 3220.

[17] S. H. Sun, C. B. Murray, D. Weller, L. Folks, A. Moser, Science 287 (2000) 1989.

[18] H. G. Bagaria, E. T. Ada, M. Shamsuzzoha, D. E. Nikles, D. T. Johnson, Langmuir 22 (2006) 7732. 


\section{Chapter 6}

\section{Transport properties of single FePt nanoparticles}

The deposition of isolated FePt magnetic nanoparticles on surfaces was achieved via the control of the rate and duration of the deposition process. Particle anchoring on flame-annealed gold substrates was achieved using 1,9-nonanedithiol self-assembled monolayers. Particle immobilization on ferromagnetic Co substrates with an $\mathrm{Al}_{2} \mathrm{O}_{3}$ tunnel barrier on top was realized with $3 \mathrm{~nm}$ poly(ethyleneimine) film as an adhesion layer. At low temperature ( $T \approx$ $40 \mathrm{~K}$ ), Coulomb blockade was observed in STM spectroscopic measurements. For a single FePt nanoparticle, a Coulomb charging energy of $150 \mathrm{meV}$ was found, in agreement with the estimated particle capacitance. Our study provides a promising measurement geometry to address isolated magnetic nanoparticles and study their (spin-dependent) transport properties. 


\subsection{Introduction}

Since the discovery of giant magnetoresistance (GMR) and tunnel magnetoresistance (TMR) in alternating ferromagnetic and non-magnetic materials [1], the field of spintronics has gained massive attention from researchers and industry alike. The GMR/TMR based devices have already been widely used in hard disk industry as read heads for years [1]. Many characteristics of GMR/TMR based devices - such as low energy consumption, nonvolatile and high sensitivity - make them attractive candidates for various applications. For instance, GMR/TMR based devices, such as magnetoresistive random access memory (MRAM) for data storage [1-2], and bio-sensors for biomedical analysis [3] are currently under development. Driven by the need for higher integration density in MRAM and TMR sensors, spintronic devices are continuously miniaturized [1]. A significant amount of interest therefore exists for the investigation of patterned media or self-assembled nanoparticle arrays.

The magnetic nanoparticles comprised of an FePt alloy, which are of particular interest because they offer high magnetocrystalline anisotropy, superior chemical stability compared to other common magnetic materials such as cobalt or iron, great flexibility in assembly, and very large scales of integration due to their reduced size [4]. Thus self-assembled monolayers of FePt nanoparticles are interesting candidates for new magnetic media, and isolated particles can be of interest in miniaturization of MRAM designs.

An FePt nanoparticle-based magnetic tunneling junction (MTJ) can potentially offer spin-valve behavior at the nanometer scale. The small dimensions involved open a new regime of behavior where new effects can be observed and harnessed. An important example of this is single-electron tunneling, which occurs when tunneling through an electrically isolated metal island of extremely small electrical capacitance. Single-electron tunneling has been reported to cause an enhancement of tunneling magneto-resistance effects, which may lead to an increase in readout signal allowing higher storage densities or operating speeds [5].

Spin-dependent transport in insulating granular films [5] and multilayers of magnetic nanoparticles with organic ligand in between [6] were studied before. 
However, spin-dependent transport studies on isolated magnetic nanoparticle are very limited [7]. Such an investigation is crucial for further miniaturizing of nanoparticle-based MRAM elements, or TMR sensors. Two major challenges in spin-dependent transport measurements on single magnetic nanoparticles are isolating/anchoring the magnetic nanoparticles and addressing magnetic nanoparticles.

In this Chapter, we propose a promising measurement geometry to study the (spin-dependent) transport properties of single FePt magnetic nanoparticles. A scanning tunneling microscope (STM) is applied to probe isolated FePt nanoparticles anchored to the substrate (Fig. 6.1). The nanoparticles were attached to flame-annealed gold films, using a 1,9-nonanedithiol self-assembled monolayer, and also to cobalt films with an $\mathrm{Al}_{2} \mathrm{O}_{3}$ tunneling barrier on top ( $\mathrm{Co} /$ $\mathrm{Al}_{2} \mathrm{O}_{3}$ ), using poly(ethyleneimine) (PEI) $[4,8]$. The inter-particle separation is tuned via the kinetics of the chemically assisted self-assembly process. Scanning tunneling microscopy was chosen since it can image the nanoparticle and access it for transport measurements [9-11]. The single-electron tunneling phenomenon, namely Coulomb blockade can be observed in a double tunnel junction system (STM tip, nanoparticle and substrate) (Fig. 6.1). As discussed in Section 2.4, it originates from the confinement of electron charge on isolated nanoparticles [12]. Two conditions should be satisfied: (1) the total capacitance of the nanoparticle coupled to the environment should be small enough that the charging energy $\frac{e^{2}}{C}$ is larger than the thermal energy $k_{\mathrm{B}} T$ and (2) the tunnel resistance of both junctions must be larger than the quantum resistance $\frac{h}{e^{2}}$ [12]. Single-electron tunneling is an important measure to verify whether the electron transport occurs through an isolated, single FePt nanoparticle. Spin-dependent transport can be studied by using a ferromagnetic Co layer with $\mathrm{Al}_{2} \mathrm{O}_{3}$ tunnel barrier on top as substrate, so that an MTJ is formed by the substrate and the isolated particle. The $\mathrm{Al}_{2} \mathrm{O}_{3}$ tunnel barrier was chosen to avoid the oxidation of the underneath Co layer, since the sample was exposed to air during nanoparticle anchoring and transportation to the STM chamber. This measurement geometry potentially allows studying nanoscale magnetoresistance, in particular the interplay with size effects such as Coulomb 
blockade.
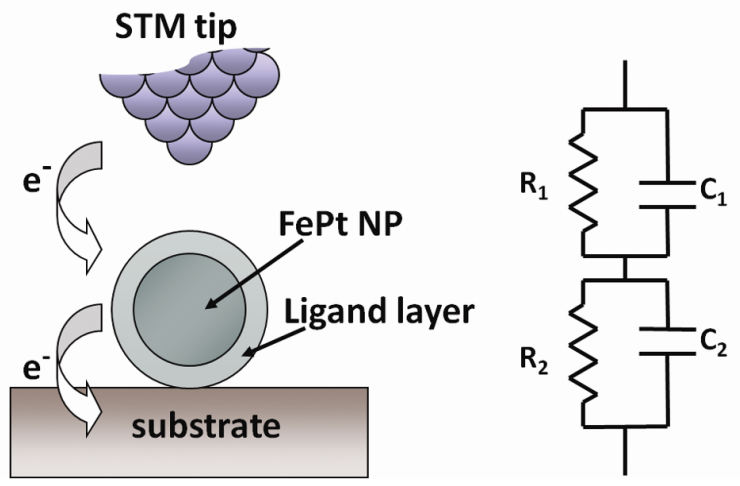

Figure 6.1: Representation of our FePt nanoparticle (NP) based double junction system and associated circuit diagram.

\subsection{FePt nanoparticles}

FePt nanoparticles were synthesized using a modification of the reported procedure by Jia et al. [13]. As-made FePt nanoparticles were stabilized by oleic acid and oleyl amine, dispersed in hexane. Transmission electron microscopy reveals that the nanoparticles have a size distribution of $3 \pm 0.5 \mathrm{~nm}$ (see Fig. 6.2). X-ray photoelectron spectroscopy (XPS) analysis on FePt nanoparticle multilayers showed an elemental composition of $\mathrm{Fe}_{0.58} \mathrm{Pt}_{0.42}$. The ratio between $\mathrm{Fe}$ and $\mathrm{Pt}$ is close to 1:1, as expected from the synthesis.
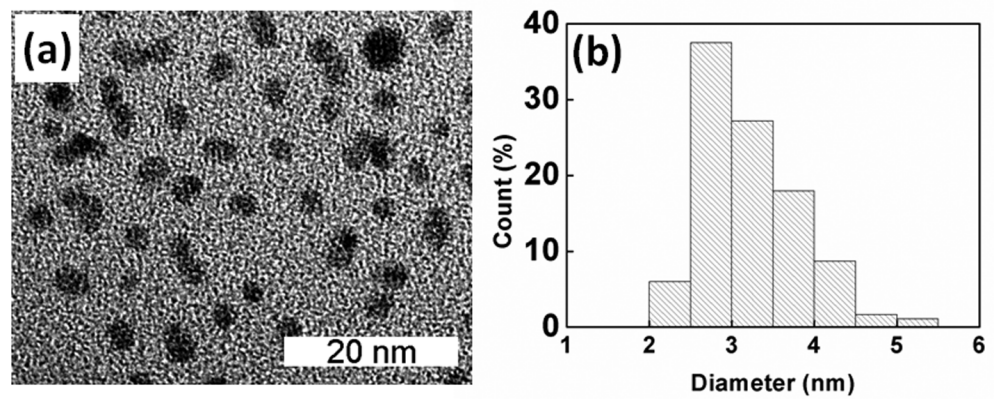

Figure 6.2: (a) Transmission electron microscope (TEM) image and (b) size histogram of FePt nanoparticles stabilized with oleic acid and oleyl amine molecules. 
The magnetic properties of (a multilayer of) these nanoparticles were measured using a vibrating sample magnetometer (VSM) at room temperature with an in-plane magnetic field (Fig. 6.3). The FePt nanoparticles are superparamagnetic at room temperature and the magnetic moment saturates at about $20 \mathrm{kOe}$. The saturation magnetic moment of this layer is about $0.2 \mu \mathrm{Am}^{2}$ at room temperature. This value was compared with the momentum density of bulk FePt $(1.14 \mathrm{~A} / \mathrm{m})$, assuming $3.0 \mathrm{~nm}$ nanoparticle radius, and $1 \mathrm{~nm}$ ligand length. The substrate dimension was $2.5 \times 10 \mathrm{~mm}$. Based on those parameters, we find a saturation magnetic moment of $0.047 \mu \mathrm{Am}^{2}$. Consequently, it is about 4 monolayers of FePt NPs on the substrate.

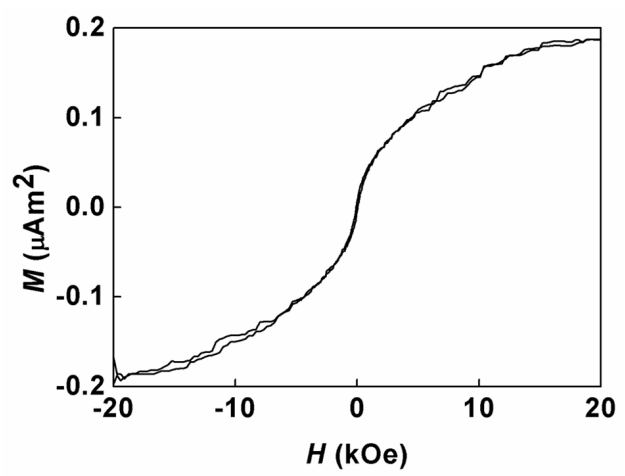

Figure 6.3: FePt nanoparticle magnetization curves measured by VSM at room temperature. The magnetic moment saturates at about $20 \mathrm{kOe}$ and the saturation magnetic moment of this layer is about $0.2 \mu \mathrm{Am}^{2}$.

\subsection{Anchoring FePt nanoparticles to the substrate}

The FePt nanoparticles were anchored to the substrate via organic linkers, which have strong affinity to both the FePt nanoparticle and the underlying substrate. In case of gold substrates, a 1,9-nonanedithiol monolayer was used [8]. A nonanedithiol SAM is approximately $1.1 \mathrm{~nm}$ thick [14]. In case of an aluminum oxide surface, a thin PEl layer was used as an adhesion layer [13]. The thickness of the PEI film is about $3 \mathrm{~nm}$ [15]. A typical nanoparticle anchoring process involved functionalization of the $\mathrm{Co} / \mathrm{Al}_{2} \mathrm{O}_{3}$ (or flame-annealed $\mathrm{Au}$ ) substrate with thin PEI layer (or nonanedithiol monolayer). Then the functionalized substrate 
was dipped in $10 \mathrm{mg} / \mathrm{ml}$ nanoparticle dispersion in hexane for $10 \mathrm{~min}$. Subsequently, it was rinsed with hexane to remove the physisorbed particles and dried in a flow of nitrogen. An STM topography image of the resulting close-packed FePt nanoparticle monolayer is shown in Fig. 6.4. Due to tip-sample convolution, the nanoparticles appear larger than the size determined by TEM (3-4 $\mathrm{nm}$ ), but the section analysis near a defect spot shows that the monolayer thickness is about $2.5 \mathrm{~nm}$. It matches well with the particle size determined by TEM. The above results were reproducible across several scans, showing that the PEI layer effectively anchors the nanoparticles.
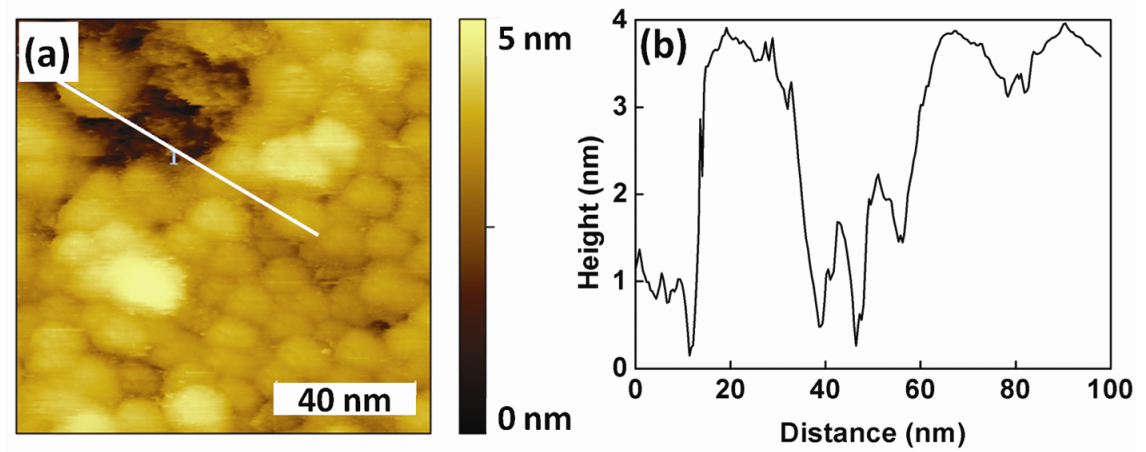

Figure 6.4: STM topography image of a FePt nanoparticle monolayer with a section analysis taken along the marked path $(I=0.5 \mathrm{nA}, V=2.75 \mathrm{~V})$.

To study transport through single FePt nanoparticles, it is essential to isolate the nanoparticles from each other. Therefore, it is preferable to have isolated, un-clustered nanoparticles instead of a close-packed monolayer of nanoparticles on the substrate. By decreasing the concentration of the nanoparticle dispersion, it is possible to reduce the amount of nanoparticles in dispersion reaches the substrate surface per unit time. A slowdown of the nanoparticle deposition rate can therefore be expected. With a moderate nanoparticle deposition rate, the density of nanoparticles on the substrate can be controlled by varying the submergence time of the substrate in the nanoparticles dispersion. The nanoparticle dispersion was diluted to $1 \mathrm{mg} / \mathrm{ml}$. PEI functionalized $\mathrm{Co} / \mathrm{Al}_{2} \mathrm{O}_{3}$ substrates were submerged in this nanoparticle dispersion for 5 and $10 \mathrm{~s}$, respectively. Atomic force microscopy (AFM) topography images indicate that $10 \mathrm{~s}$ 
dipping results in a loosely packed nanoparticle monolayer, and $5 \mathrm{~s}$ dipping results in a lower nanoparticle density (Fig. 6.5). These results illustrate the very high sensitivity of the nanoparticle deposition to the submergence time.
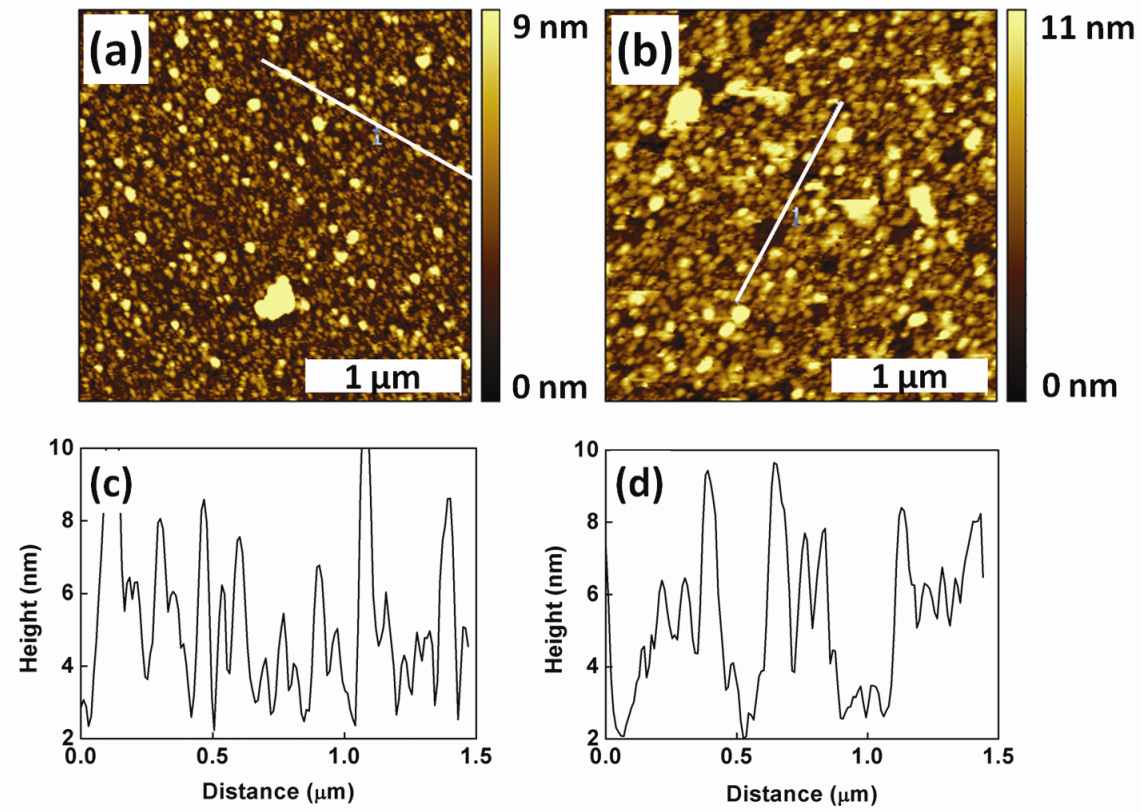

Figure 6.5: AFM topography scans of FePt nanoparticles deposited on a PEI functionalized $\mathrm{Co} / \mathrm{Al}_{2} \mathrm{O}_{3}$ substrate from $1 \mathrm{mg} / \mathrm{ml}$ nanoparticle dispersion. The submergence time is $5 \mathrm{~s}(\mathrm{a})$ and $10 \mathrm{~s}(\mathrm{~b})$.

The nanoparticle dispersion was further diluted to $0.5 \mathrm{mg} / \mathrm{ml}$. This slowed down the nanoparticle deposition rate, thus resulting in better control of the nanoparticle density on the substrate. Figure 6.6a shows the result of $300 \mathrm{~s}$ submergence using this concentration, revealing about $3 \mathrm{~nm}$ features that strongly suggest isolated single particle deposition. This notion is enforced by a significant decrease in the number of agglomerations compared to previous results. A further decrease in particle density was also possible, as illustrated by the 60 and $15 \mathrm{~s}$ submergence samples (Figs. $6.6 \mathrm{~b}$ and c, respectively). This shows that a dispersion concentration of $0.50 \mathrm{mg} / \mathrm{ml}$ is low enough to limit the rate of the nanoparticle deposition by reducing the amount of nanoparticle diffusion to the substrate surface per unit time. 

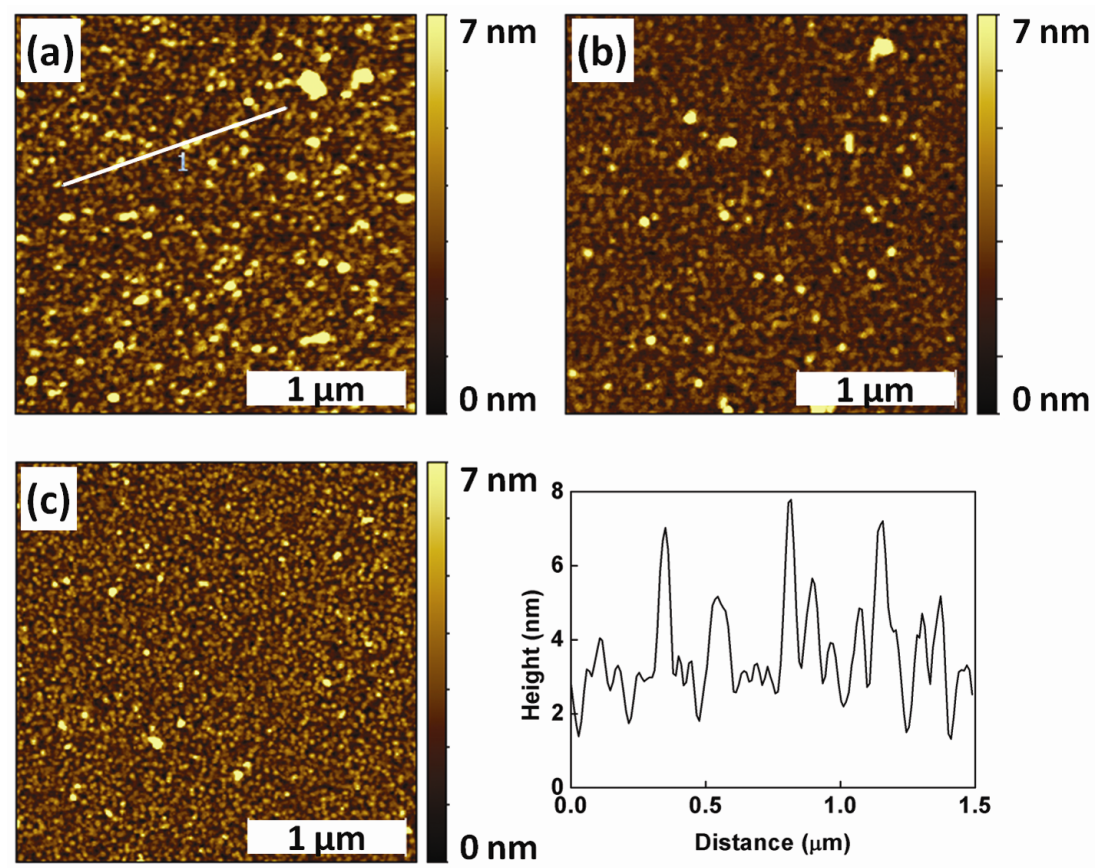

Figure 6.6: AFM topography scans of FePt NPs deposited on a PEI functionalized Co/ $\mathrm{Al}_{2} \mathrm{O}_{3}$ substrate. The concentration of the NPs dispersion is $0.50 \mathrm{mg} / \mathrm{ml}$. The submergence time is $300 \mathrm{~s}(\mathrm{a}), 60 \mathrm{~s}$ (b) and $15 \mathrm{~s}$ (c). The height profile (d) shows isolated features about 3 $\mathrm{nm}$ in height.

Similar experiments were carried out with flame-annealed Au substrates functionalized with nonanedithiol SAMs. Isolated single FePt nanoparticles anchored on flame annealed Au substrate were obtained by using a $0.50 \mathrm{mg} / \mathrm{ml}$ nanoparticle dispersion and 60 s submergence time.

\subsection{Single-electron tunneling through isolated FePt nanoparticles}

FePt nanoparticles anchored to flame-annealed gold surfaces with nonanedithiol were studied with STM. Two room-temperature STM images are shown in Fig. 6.7. These scans were taken at relatively low bias voltage and set point current (50 pA) in order to increase the tip-sample separation, which prevents damage to the dithiol SAM during imaging and avoids the tip-nanoparticle interaction that 
might drag the nanoparticle on the SAM surface. The spotted pattern on the surface is typical for dithiol monolayers on gold and has been identified as monatomic steps in the flame-annealed Au substrate, rather than imperfections in the dithiol SAM. The formation of these single-step holes in the flame-annealed $\mathrm{Au}$ substrate is thought to be caused by an etching effect inherent to the molecular self-assembly processes of thiol adsorption [16]. When taking the cross-sectional profile along one such structural feature (Fig. 6.7d, profile 1), the depth indeed equals an Au atomic step (Fig. 6.7c, profile 1) $(d \approx$ $0.25 \mathrm{~nm}$ ). Nanoparticles were visible in both scans of Fig. 6.7a and b. In the case of Fig. 6.7b the nanoparticle remained stationary during multiple successive scans, showing that it was firmly attached to the dithiol SAM. As shown in Fig. 6.7a however, other nanoparticles were not so well anchored and were dragged across the surface by the STM tip.
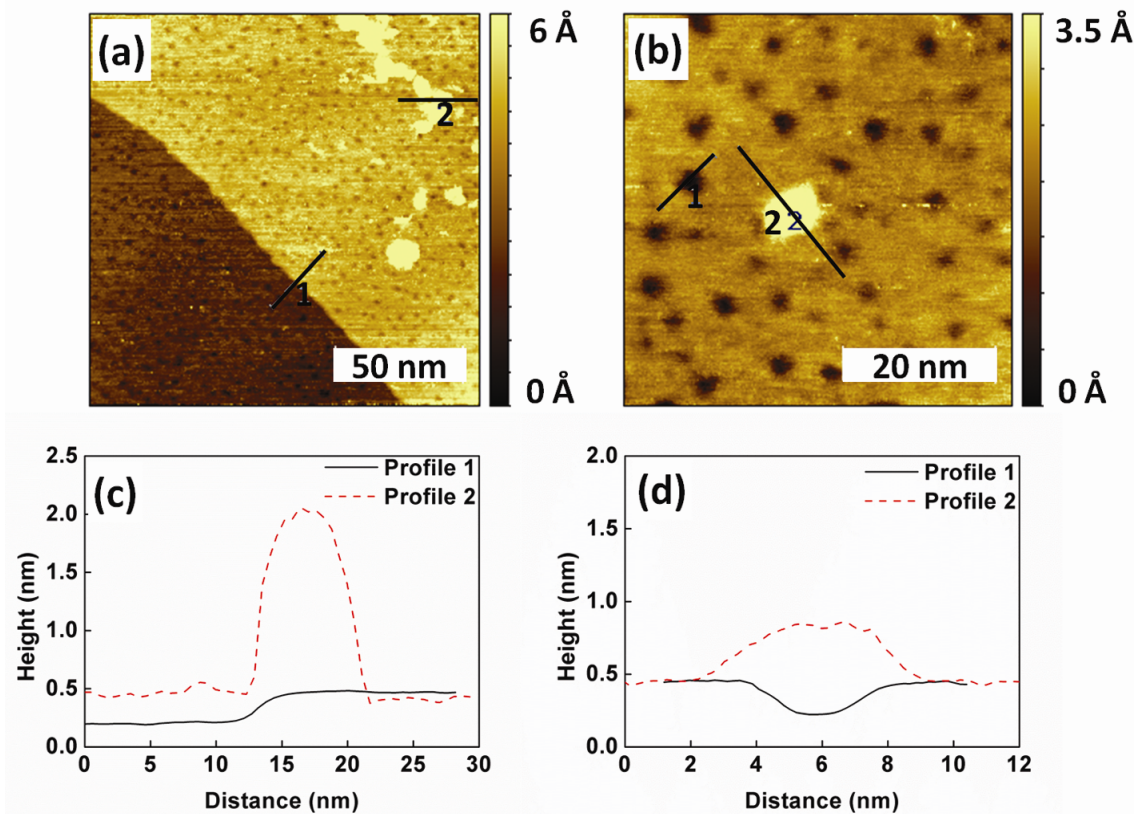

Figure 6.7: STM topography images and height profiles of nanoparticles on a nonanedithiol monolayer on gold ( $V=0.1 \mathrm{~V}, I=50 \mathrm{pA})$. (a) Monoatomic step of Au with a nanoparticle dragged across the surface by the STM tip. (b) Close-up of a strongly anchored nanoparticle. The line profiles (c) and (d) were taken along the marked paths in (a) and (b). 
It is interesting to note that the height profile of the stationary nanoparticle is only about $0.4 \mathrm{~nm}$, whereas the measured height of the moving particle amounts to more than $1.5 \mathrm{~nm}$. This, together with the fact that the expected particle diameter is around $3 \mathrm{~nm}$, suggests that the anchored particle is embedded in the dithiol layer. When a nanoparticle is partially buried in the monolayer, for example at one of the dithiol SAM depression sites, it strongly increases the chance of multiple thiol molecules being bound to the nanoparticle surface, and hence the anchoring becomes much stronger.

Repeating the experiment at low temperature led to the same results. The topography images in Fig. 6.8a were performed at $T \approx 40 \mathrm{~K}$ and show a strongly anchored nanoparticle, with a measured height of about $0.8 \mathrm{~nm}$. Spectroscopic measurements were performed on this FePt nanoparticle at $T \approx 40 \mathrm{~K}$. The spectroscopic curves in Fig. 6.8b show clear Coulomb blockade behavior, with the slope at zero voltage virtually flat (i.e. blockade). Apart from the Coulomb blockade around $\mathrm{OV}$, also the Coulomb staircase was observed. Repeated on-particle I-V spectra were shown in Fig. 6.9a together with a $d I / d V$ plot (Fig. 6.9b). At every plateau (Fig. 6.9a), the number of electrons that can tunnel spontaneously is constant. When the bias is high enough to overcome the charging energy of the nanoparticle, more electrons can tunnel at a time. It results in the step wise $I-V$ characteristics.
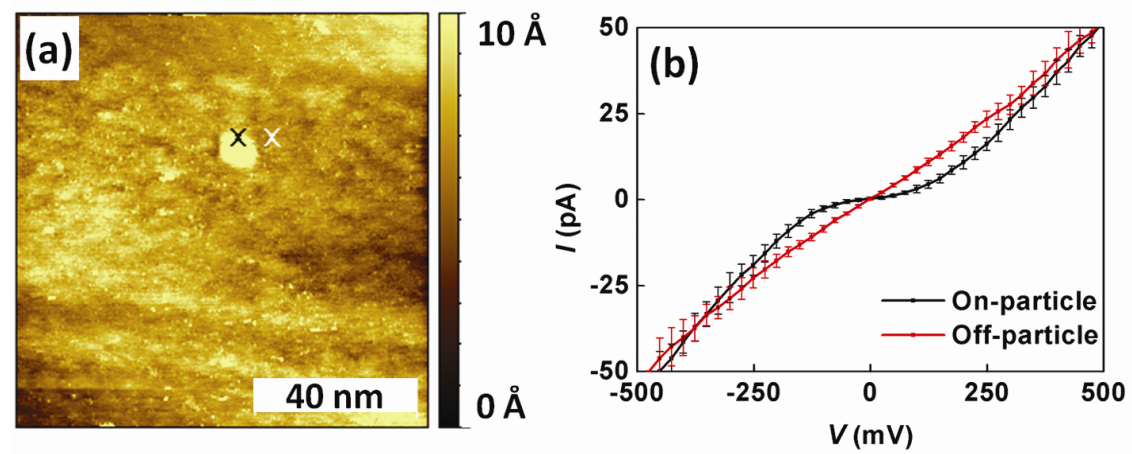

Figure 6.8: Low-temperature STM image of FePt nanoparticles on flame-annealed gold with nonanedithiol SAM ( $I=50 \mathrm{pA}, V=0.5 \mathrm{~V}, T \approx 40 \mathrm{~K}$ ) (a), with $I-V$ spectroscopy measurements performed at the marked locations ( 5 averages) (b). 
The Coulomb staircase allows us to extract and estimate the Coulomb charging energy of the nanoparticle. The peaks in the $\mathrm{d} / / \mathrm{d} V$ plot of Fig. 6.9 are spaced approximately $150 \mathrm{mV}$ apart. At these peaks the conductivity is high, meaning that the tunneling electrons have enough energy to overcome an extra unit of charging energy $e^{2} / C$. This allows the conduction to go from the single-electron tunneling (SET) regime to the double-electron tunneling regime, then to triple-electron tunneling, and so on. The estimated Coulomb charging energy of the nanoparticle is about $150 \mathrm{meV}$, which is very reasonable when compared to the charging energy of $\sim 140 \mathrm{meV}$ estimated for a $3 \mathrm{~nm}$ nanoparticle placed on top of the nonanedithiol [17].
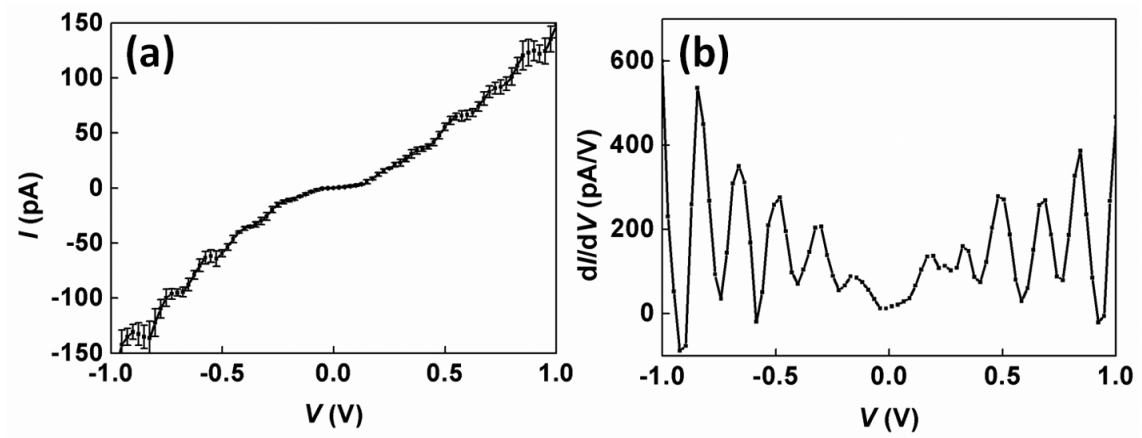

Figure 6.9: On-particle $I-V$ spectrum of the FePt nanoparticle shown in Fig. 6.8a at $T \approx$ $40 \mathrm{~K}(\mathrm{a})$, plotted with its 3-points numerical derivative (b). The peaks in the $d / / d V$ plot are spaced approximately $150 \mathrm{mV}$ apart.

During the course of the STM experiments, the Coulomb blockade was observed at $T \approx 40 \mathrm{~K}$ on more than ten different nanoparticles. In all cases the result was reproducible by immediately repeating the measurement, and identical results were obtained for over 10 repeat measurements on some nanoparticles. The Coulomb blockade could be readily achieved at cryogenic temperatures for almost all nanoparticles found by STM imaging. Reproducible Coulomb blockade obtained on isolated FePt nanoparticles on flame-annealed Au surface proves the ability to use STM access and measure the transport properties of isolated single FePt nanoparticles. 


\subsection{STM study on isolated FePt NPs on $\mathrm{Co} / \mathrm{Al}_{2} \mathrm{O}_{3} / \mathrm{PEI}$ surface}

To investigate the spin-dependent transport in isolated FePt nanoparticles, a promising measurement geometry was proposed consisting of a STM and samples with isolated FePt nanoparticles deposited on a ferromagnetic substrate (Fig. 6.10). This forms a magnetic tunnel junction like geometry. The ferromagnetic $\mathrm{Co}$ thin film $(10 \mathrm{~nm})$ with $\mathrm{Al}_{2} \mathrm{O}_{3}$ tunnel barrier $(2.5 \mathrm{~nm})$ was chosen as substrate. The $\mathrm{Al}_{2} \mathrm{O}_{3}$ tunnel barrier protected the $\mathrm{Co}$ layer from oxidation during transportation in air. As discussed in Section 6.3, the isolated FePt nanoparticles were attached to the $\mathrm{Al}_{2} \mathrm{O}_{3}$ surface via PEI layer. TMR experiments can be performed by switching the relative magnetic orientation of ferromagnetic substrate and the magnetic nanoparticle by an external magnetic field while monitoring the tunnel resistance (Fig. 6.10).

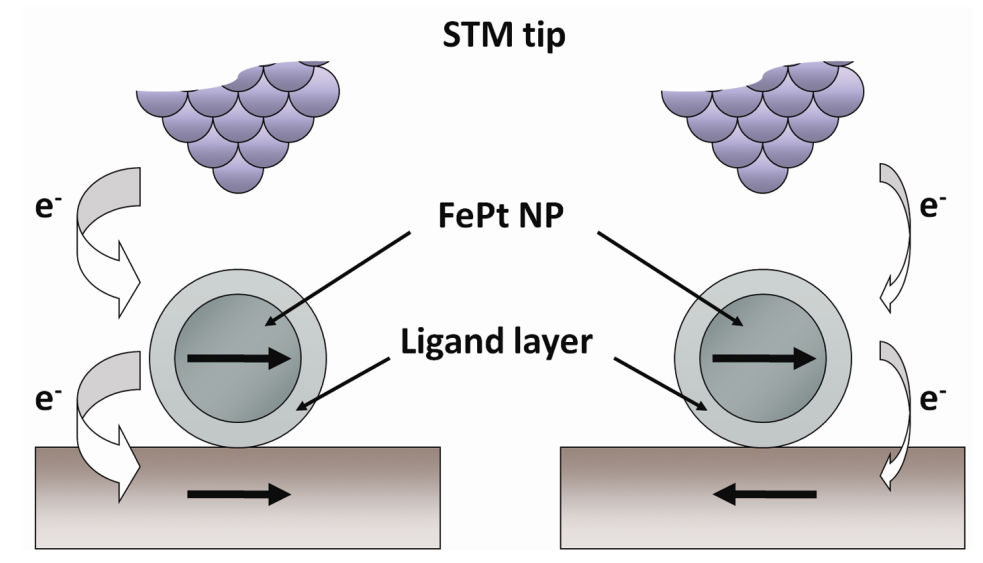

Figure 6.10: Electron tunneling through an MTJ structure formed by an STM tip, an isolated magnetic FePt nanoparticle and a magnetic substrate.

In order to facilitate the (spin-dependent) transport studies, STM imaging on samples with isolated FePt nanoparticles on $\left(\mathrm{Co} / \mathrm{Al}_{2} \mathrm{O}_{3} / \mathrm{PEI}\right)$ surface was carried out. Figure 6.11 shows the result of a surface scan performed with very low current setpoint and at high voltage, in order to keep a large distance between sample and STM tip. Despite these settings the image appears quite blurry, which is evidence of a considerably poor tip quality caused either by the tip crashing into the surface or contaminations being picked up by the tip. The large height of 
the two protruding features shows that they are probably agglomerations of nanoparticles.
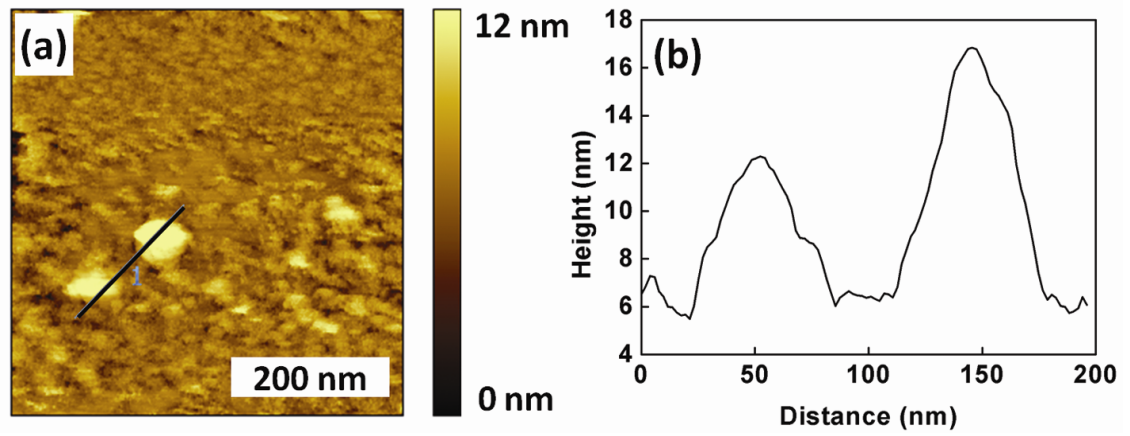

Figure 6.11: Room-temperature STM topography image of a $\mathrm{Co} / \mathrm{Al}_{2} \mathrm{O}_{3} / \mathrm{PEI}$ surface with two (agglomerations of) nanoparticles visible $(I=50 \mathrm{pA}, V=3.0 \mathrm{~V})$.

The difficulty of maintaining a good tip quality on these $\mathrm{Co} / \mathrm{Al}_{2} \mathrm{O}_{3} / \mathrm{PEI}$ samples is very likely caused by the thick tunneling barrier $\left(\mathrm{Al}_{2} \mathrm{O}_{3}\right.$ and $\left.\mathrm{PEI}\right)$ covering the cobalt film. Due to this high tunneling resistance the STM tip has to come very close to the surface. This increases the risk of the tip making physical contact with the surface when it encounters a high topographic feature during scanning. This issue was found to be even more problematic when cooling the system to cryogenic temperatures, where tip crashing could be evidenced during approaching or while scanning. This temperature dependence indicates that the conduction through the $\mathrm{Al}_{2} \mathrm{O}_{3} / \mathrm{PEI}$ barrier is dominated by thermally activated hopping. The lower thermal energy leads to an increase in the total resistance and forces the feedback loop to further decrease the tip-sample separation, causing the tip to make physical contact with the substrate. The spin-dependent transport study was thus not possible due to the ease of degradation of the STM tip on $\mathrm{Co} / \mathrm{Al}_{2} \mathrm{O}_{3} / \mathrm{PEI}$ surface.

\subsection{Conclusion}

In conclusion, the isolation of FePt nanoparticles on $\mathrm{Au}$ and $\mathrm{Co} / \mathrm{Al}_{2} \mathrm{O}_{3}$ surfaces was investigated and achieved. The results were verified by STM topographic 
measurements. Using STM I-V spectroscopy, Coulomb blockade in more than ten different FePt nanoparticles on a flame-annealed Au / nonanedithiol SAM surface was observed reproducibly at low temperatures $(T \approx 40 \mathrm{~K}$ ), and clear Coulomb staircase was measured. Using the latter result the Coulomb charging energy of the nanoparticles was found to be approximately $150 \mathrm{meV}$. This result is in good agreement with the estimated charging energy of a $3 \mathrm{~nm}$ nanoparticle.

Room-temperature and low-temperature STM topography imaging of nanoparticles on $\mathrm{Co} / \mathrm{Al}_{2} \mathrm{O}_{3} / \mathrm{PEI}$ substrates was found to be challenging due to severe tip degradation. This is most probably caused by the tip approaching too closely to the surface, trying to overcome the high tunneling resistance of the $\mathrm{Al}_{2} \mathrm{O}_{3} / \mathrm{PEI}$ tunnel barrier. Further work on anchoring nanoparticles with less resistive monolayer on magnetic substrates is under way. This would potentially allow us investigate the spin dependent transport through those nanoparticles.

\subsection{Experimental section}

Surfactant stabilized FePt nanoparticles were synthesized via a modified method reported by Sun et al. [1]. A solution of $0.25 \mathrm{mmol} \mathrm{Pt}(\mathrm{acac})_{2}$ and $0.75 \mathrm{mmol}$ 1,2-hexadecanediol in $20 \mathrm{~mL}$ octyl ether was heated to $80^{\circ} \mathrm{C}$, and to this solution $0.5 \mathrm{mmol}$ oleic acid, $0.5 \mathrm{mmol}$ oleyl amine and $0.5 \mathrm{mmol} \mathrm{Fe}(\mathrm{CO})_{5}$ were added via a syringe under a fume hood. Caution: the decomposition of $\mathrm{Fe}(\mathrm{CO})_{5}$ produces $\mathrm{CO}$, which is potentially lethal. The mixture was further heated to $150^{\circ} \mathrm{C}$ for $1 \mathrm{~h}$. The black product was precipitated using ethanol and the particles were redispersed in hexane. This procedure was reported to yield a 1:1 Fe:Pt ratio in the NPs.

X-Ray photoelectron spectroscopy (XPS): Elemental composition was analyzed by a Physical Electronics Quantera Scanning X-ray Multiprobe instrument, equipped with a monochromatic Al K $\alpha$ X-ray source operated at $1486.7 \mathrm{eV}$ and $25 \mathrm{~W}$. Spectra were referenced to the main C1s peak at $284.80 \mathrm{eV}$.

Vibrating Sample Magnetometer (VSM): Magnetic studies were carried out using a DMS vibrating sample magnetometer (model VSM10) with fields up to $1500 \mathrm{kA} / \mathrm{m}$ and a sensitivity of $10^{-6} \mathrm{mAm}^{2}$. 
The flame-annealed gold substrates were prepared from a $200 \mathrm{~nm}$ thick sputtered gold film on top of glass substrates. These substrates were heated in a hydrogen flame for $5 \mathrm{~min}$ and then allowed to cool down to room temperature in air for $1 \mathrm{~min}$. Immediately after cooling the samples are submerged in a dithiol solution to prevent surface contaminations from prolonged exposure in air.

Freshly prepared flame annealed gold substrate was submerged in a $3 \mathrm{mM}$ solution of 1,9-nonanedithiol in ethanol. The monolayer formation was under an argon atmosphere for $17 \mathrm{hr}$. Then the samples were rinsed with ethanol and dried under $\mathrm{N}_{2}$ flow.

To prepare the $\mathrm{Co} / \mathrm{Al}_{2} \mathrm{O}_{3}$ substrate for deposition of a PEI layer, it was first ultrasonically cleaned in acetone and isopropyl alcohol (10 min each) and treated with oxygen plasma to activate the surface ( $2 \mathrm{~min}$ at $0.25 \mathrm{mbar}$ pressure, $18 \% \mathrm{O}_{2}$ flow and $300 \mathrm{~W}$ power). It was then submerged for $5 \mathrm{~min}$ in a $20 \mathrm{mg} / \mathrm{ml}$ solution of PEI in chloroform, after which the surface was through rinsed with ethanol and dried under $\mathrm{N}_{2}$ flow.

An ultrahigh vacuum (UHV) low-temperature STM was used to perform the topography and spectroscopy measurements with a PtIr tip. The measurements were done at room temperature and at about 40K. The IV spectra were taken at pre-defined positions during the STM imaging with the feedback loop open.

\section{References}

[1] J. G. J. Zhu, C. D. Park, Materials Today 9 (2006) 36.

[2] S. Tehrani, J. M. Slaughter, E. Chen, M. Durlam, J. Shi, M. DeHerrera, IEEE Transactions on Magnetics 35 (1999) 2814.

[3] G. Mihajlovic, S. von Molnar, Solid-State Magnetic Sensors for Bioapplications Springer (2009)

[4] S. Sun, C. B. Murray, D. Weller, L. Folks, A. Moser, Science 287 (2000) 1989.

[5] K. Yakushiji, S. Mitani, F. Ernult, K. Takanashi, H. Fujimori, Physics Reports-Review Section of Physics Letters 451 (2007) 1. 
[6] S. Wang, F. J. Yue, J. Shi, Y. J. Shi, A. Hu, Y. W. Du, D. Wu, Applied Physics Letters 98 (2011)

[7] M. M. Deshmukh, S. Kleff, S. Gueron, E. Bonet, A. N. Pasupathy, J. von Delft, D. C. Ralph, Physical Review Letters 87 (2001) art. no.

[8] R. P. Andres, T. Bein, M. Dorogi, S. Feng, J. I. Henderson, C. P. Kubiak, W. Mahoney, R. G. Osifchin, R. Reifenberger, Science 272 (1996) 1323.

[9] T. Ohgi, D. Fujita, Surface Science 532 (2003) 294.

[10]D. Anselmetti, T. Richmond, A. Baratoff, G. Borer, M. Dreier, M. Bernasconi, H. J. Guntherodt, Europhysics Letters 25 (1994) 297.

[11] C. Schonenberger, H. Vanhouten, H. C. Donkersloot, Europhysics Letters 20 (1992) 249.

[12] U. Meirav, E. B. Foxman, Semiconductor Science and Technology 11 (1996) 255.

[13] Z. Y. Jia, S. S. Kang, D. E. Nikles, J. W. Harrell, IEEE Transactions on Magnetics 41 (2005) 3385.

[14] P. Kohli, K. K. Taylor, J. J. Harris, G. J. Blanchard, Journal of the American Chemical Society 120 (1998) 11962.

[15] O. Yildirim, T. Gang, S. Kinge, D. N. Reinhoudt, D. H. A. Blank, W. G. van der Wiel, G. Rijnders, J. Huskens, International Journal of Molecular Sciences 11 (2010) 1162.

[16] C. Schoenenberger, J. A. M. Sondag-Huethorst, J. Jorritsma, L. G. J. Fokkink, Langmuir 10 (1994) 611.

[17] C. Gao, F. Duewer, X. D. Xiang, Applied Physics Letters 75 (1999) 3005. 


\section{Chapter 7}

\section{Towards hybrid organic-inorganic electron interferometers}

In this chapter, an investigation on electron interferometers for the final purpose of studying the degree of coherency of electron transport through organic molecules is described. Several different electron interferometers were designed and fabricated by e-beam lithography. Modified electron interferometers allow the insertion of molecular monolayers in the arms via nanoparticle bridging. This offers a potential platform for studying the coherency of electron transport through molecules, which has rarely been addressed in the research community so far. Coherency can be a valuable asset in molecular electronics, e.g. in the context of quantum computation. Aharonov-Bohm (AB) oscillations were observed using a cryogenic setup and customized low-noise measurement electronics. The effect of the device geometry, temperature and the excitation current on the $A B$ oscillations was studied. 


\subsection{Introduction}

The flexibility and versatility offered by organic molecules make them attractive candidates for building blocks of future electronic devices. Coherent transport in organic-inorganic hybrid systems down to the molecular level has rarely been addressed in the research community so far. Coherency may be a valuable asset in molecular electronics, e.g. in the context of quantum computation.

Coherent transport implies that an electron preserves its phase information over a certain distance, the phase coherence length $\left(l_{\varphi}\right)$. When an electron propagates through a molecule, parameters like the nature of the chemical bonding, length and composition may affect the phase coherence. In this chapter, we discuss a device geometry, which can be used to explore the coherency of electron transport in molecules.

$A$ ring-shaped $A B$ electron interferometer ( $A B$ ring) was chosen as a probe for coherent electron transport [1-3]. Two different device geometries were designed. The standard $A B$ electron interferometer was used to examine and fine tune the measurement setup (Fig. 7.1a). The modified $A B$ electron interferometer allows the insertion of molecular monolayers into the circuit via nanoparticle bridging (Figs. 7.1b and c) [4-6]. A nano gap was defined in one arm of the $A B$ ring for the nanoparticle bridging. A resistive junction was made in the other arm of the $A B$ ring to balance the tunnel resistance of the molecular junction.

As described in section 2.5, when a magnetic field is swept perpendicular to the $A B$ ring, resistance oscillations can be measured due to the interference between two partial electron waves which travel through the two arms of the $A B$ ring. If the inserted molecules cause any decoherence (Fig. 7.1b), it could (partially) destroy the interference, thus (partially) wash out the resistance oscillations.

NP bridging is a reliable and robust way to insert molecular monolayers into a conduction path. A typical procedure of nanoparticle bridging consists of the following steps. First, a self-assembled monolayer of the target molecule is formed on a pair of electrodes with a nano gap in between. Then a gold nanoparticle can be trapped in the gap via various techniques [4-6]. 
Consequently, the gap between two electrodes is bridged by the gold nanoparticle (Fig. 7.1c).

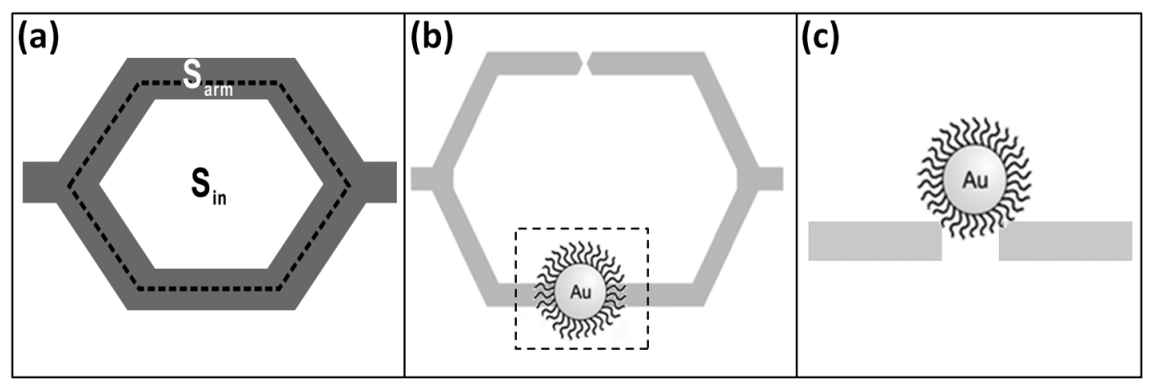

Figure 7.1: Schematic drawing of an $A B$ ring (a) and a modified $A B$ ring (b). The nano gap is bridged by an Au nanoparticle with ligands around it. (c), cross section diagram of the dashed area in (b).

\subsection{Device design and fabrication}

The $A B$ effect in a ring (with a circumference $L$ ) can be observed only if electrons can keep their phase information when traveling along that ring. In other words, the condition $l_{\varphi}>\sim L / 2$ should be satisfied. Cooling down the device can dramatically increase the coherence length due to reduced electron phonon interactions [7]. In this experiment, gold is used for the ring, which has a phase coherence length of $\sim 1 \mu \mathrm{m}$ at our base temperature of $250 \mathrm{mK}$ [8-9]. This sets a tight design requirement for the device $\frac{L}{2}<1 \mu \mathrm{m}$.

As described in Chapter 2, the magnitude of $A B$ oscillations and universal conductance fluctuations (UCF) both are of order $e^{2} / h$. The magnetic field period of the $\mathrm{AB}$ oscillation is determined by the area enclosed by the ring $\left(S_{i n}\right)$, whereas the correlation field of UCF is correlated with the area of the arms $\left(S_{\text {arm }}\right)$. The ratio $S_{\text {in }}: S_{\text {arm }}$ is called the aspect ratio of the ring (Figure 7.1a). If the aspect ratio is small, the magnetic field period of the $A B$ oscillations and the correlation field of the UCF are of the same order. So the $A B$ oscillations will be overshadowed by UCF and cannot be distinguished. $\frac{S_{\text {in }}}{S_{\text {arm }}} \gg 1$ is needed for a measureable $\mathrm{AB}$ oscillation. The maximal $S_{\text {in }}$ is limited by $\frac{L}{2}<l_{\varphi}$. So, the area 
of the arms $\left(S_{\text {arm }}\right)$ needs to be as small as possible to observe clear $\mathrm{AB}$ oscillations. This means a narrow arm width (tens of nanometers) is required.

\subsubsection{Device design}

The dimension requirements discussed above are beyond the resolution of optical lithography. E-beam lithography was chosen since its resolution can go down to tens of nanometers. The e-beam lithography process of the samples was completed by Mr. Yoshida at Prof. Seigo Tarucha's lab at the NTT Basic Research Labs. There were mainly three types of e-beam structures in the design file. They are denoted as hexagonal $A B$ rings, line with gap and resistive junction. The diameter of the rings is varied from $300 \mathrm{~nm}$ to $1000 \mathrm{~nm}$. The arm width is $40 \mathrm{~nm}$. The gap size is varied between 0 to $80 \mathrm{~nm}$. Tests can be run on those nano gaps to find out an optimal gap size for the nanoparticle bridging. Resistive junctions with narrow constrictions were made to vary the transmission factor of the conduction path. A detailed design map of the e-beam structures is given in Appendix A.

\subsubsection{Device fabrication}

Samples $(10 \mathrm{~mm} \times 10 \mathrm{~mm}$ ) were prepared on a standard p-type <100> silicon wafer with $40 \mathrm{~nm}$ thermally grown $\mathrm{SiO}_{2}$ on top. The spot size of the electron beam on resist layer can reach 3-5 $\mathrm{nm}$. After exposure and development, the corresponding feature size is about $10 \mathrm{~nm}$. The beam dwell time was tested and fixed at 1.9 microseconds. Afterwards, the pattern was converted into a gold structure by a metal lift-off process. The metal layer consists of a thin $10 \mathrm{~nm} \mathrm{Ti}$ adhesion layer and $40 \mathrm{~nm}$ Au. The e-beam structures are too small for wire bonding. Therefore, an optical mask was designed for large-scale contact pads. The mask layout for the contact pads can be found in Appendix B. Standard optical lithography was carried out with ODP 907/17 positive photoresist. Metal deposition and lift-off were used to make the large-scale electrodes (100 nm Ti / $500 \mathrm{~nm} \mathrm{Au}$ ). 


\subsubsection{SEM inspection}

The e-beam structures were inspected by a scanning electron microscope (SEM). All the SEM images were taken at $5 \mathrm{kV}$ acceleration voltage and $98 \mathrm{pA}$ beam current. In Fig. 7.2a, a complete ring can be seen, which has $604 \mathrm{~nm}$ diameter and $28 \mathrm{~nm}$ arm width. According to the design (Appendix A), the desired diameter of the ring and arm are $600 \mathrm{~nm}$ and $40 \mathrm{~nm}$, respectively. The actual diameter agrees with the design value. The arm width is narrower than the design value. However, a reduced width means larger aspect ratio; this may lead to a more clearly visible $A B$ oscillation (see section 2.5).

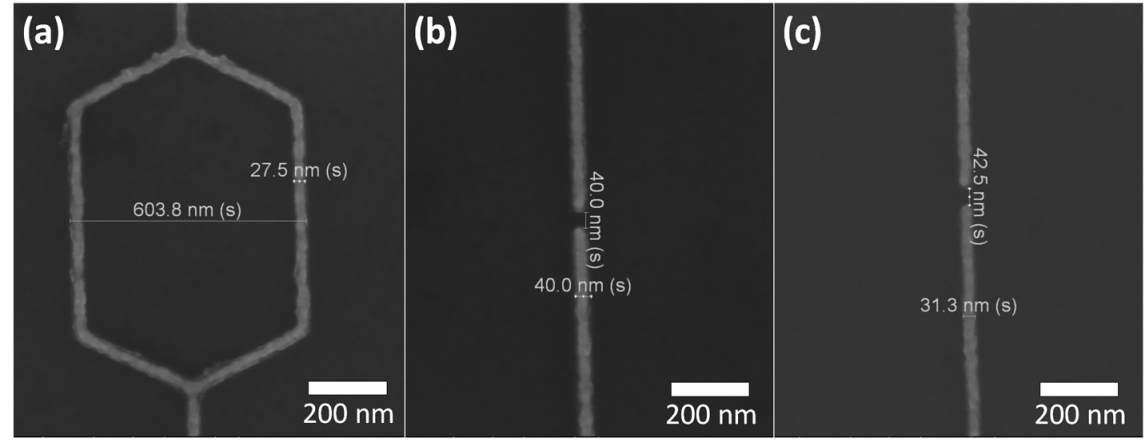

Figure 7.2: SEM images of a $600 \mathrm{~nm}$ width ring (H12) (a), line with gap structure (M12) (b), junction with spacing structure (G12) (c).

A line with gap structure is shown in Fig. 7.2b. In this case, the designed and actual gap size are consistent, both $40 \mathrm{~nm}$. One of the examples of a resistive junction structure is presented in Fig. 7.2c. It is clear that the desired narrow constriction in the middle of the junction structure has not been achieved. In future devices, modification of the e-beam design is needed to improve such structures.

\section{3 ${ }^{3} \mathrm{He}$ refrigerator and device cooling}

Temperatures down to hundreds of millikelvin are required to measure the $A B$ effect, since $l_{\varphi}$ is long enough in that regime. Such low temperatures are reached with the Oxford Instruments Heliox VL, which is a closed-cycle ${ }^{3} \mathrm{He}$ 
cryostat with a base temperature of $230 \mathrm{mK}$. The maximum hold time is about 70 hours. A superconducting magnet that can go up to $10 \mathrm{~T}$ is also included in this cryostat.

The sample is loaded into an internal vacuum can (IVC) in order to shield it from the liquid ${ }^{4} \mathrm{He}$ bath at $4 \mathrm{~K}$. There are two stages of operation of the Heliox $\mathrm{VL}-$ condensation and cooling. In Fig. 7.3, these two stages are shown. The condensation is done by heating up the ${ }^{3} \mathrm{He}$ sorption pump to about $32 \mathrm{~K}$; at this temperature, it will release all the ${ }^{3} \mathrm{He}$ gas adsorbed. This gas is condensed at the $1 \mathrm{~K}$ plate and collected in the ${ }^{3} \mathrm{He}$ pot. In the cooling stage, the sorption pump is cooled; it will start to absorb ${ }^{3} \mathrm{He}$ gas and effectively decrease the ${ }^{3} \mathrm{He}$ gas pressure above the liquid ${ }^{3} \mathrm{He}$. Due to the evaporation of ${ }^{3} \mathrm{He}$ under reduced pressure, the temperature in the ${ }^{3} \mathrm{He}$ pot is possible to reach $230 \mathrm{mK}$.

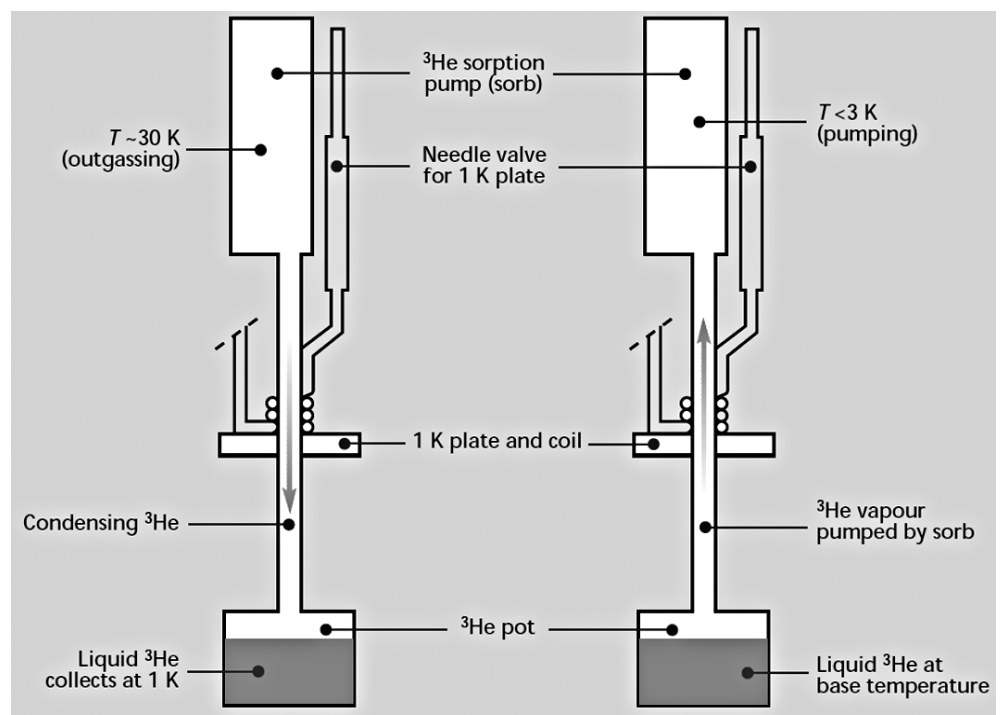

Figure 7.3: Condensation and Cooling mechanism of Heliox [10].

The cold finger is physically and thermally connected to the ${ }^{3} \mathrm{He}$ pot (Fig. 7.4a). Two sample carriers were fabricated for magnetic field parallel or perpendicular to the sample. The maximal sample size is $11 \times 11 \mathrm{~mm}$. The sample carrier mounts on the cold finger and has 20 bonding pads for accessing the sample (Figs. 7.4b and c). Looms of copper DC wires are thermally anchored to the cold finger and connected to the sample via the sample carrier. The samples are 
cooled through these DC wires. In addition, the back side of the sample is also thermally anchored to the cold finger via the sample carrier. So the sample is also cooled from the back. Although the phonon temperature will be close to the base temperature of the cryostat, the electron temperature can be higher, as the electron temperature is affected by high-frequency radiation or noise in the DC wires. To suppress these effects, SMD (surface mount device) capacitors were mounted on the back side of the sample carrier. The capacitance is $47 \mathrm{nF}$ and the resistance of the wire is about $240 \mathrm{Ohm}$. A cut-off frequency of $14 \mathrm{kHz}$ is obtained.

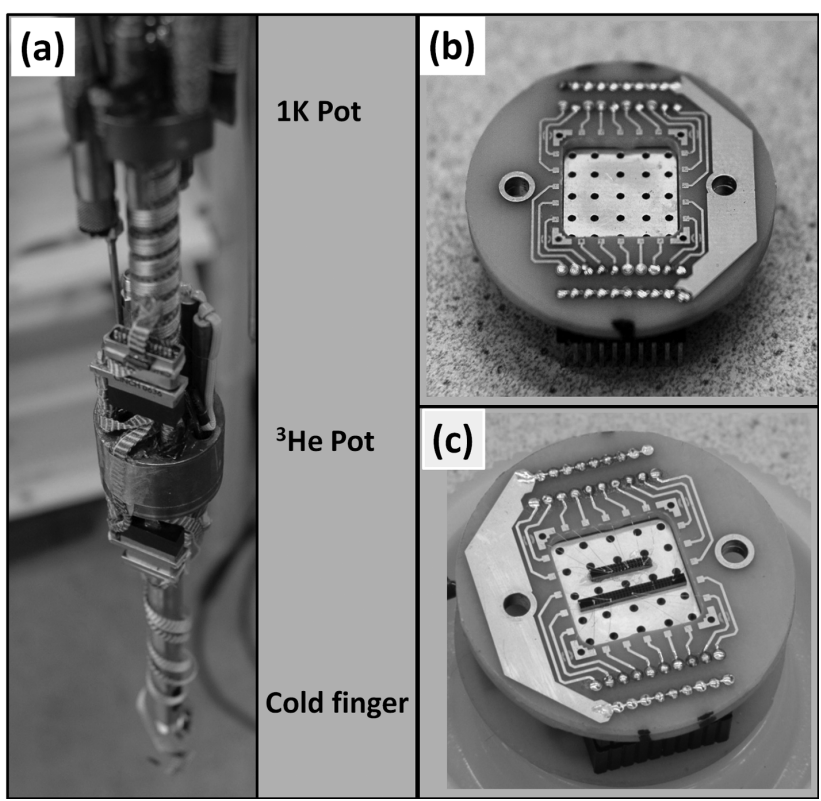

Figure 7.4: Image of cold finger (a), empty sample carrier (b) and sample carrier with device (c).

\subsection{Measurement electronics and wiring}

Typical measurements on the $A B$ electron interferometer at low temperature consist of probing the resistance of the device as a function of magnetic field. The architecture of the circuit is given in Fig. 7.5. The electronics connect directly to the sample (current source, voltage amplifier) contain isolation amplifiers. This protects the sample from the voltage spikes generated by the switching elements 
in the external measurement instruments. Furthermore all elements connect directly to the sample is purely analog (no clock generator) and battery driven (prevent $50 \mathrm{~Hz}$ interference).

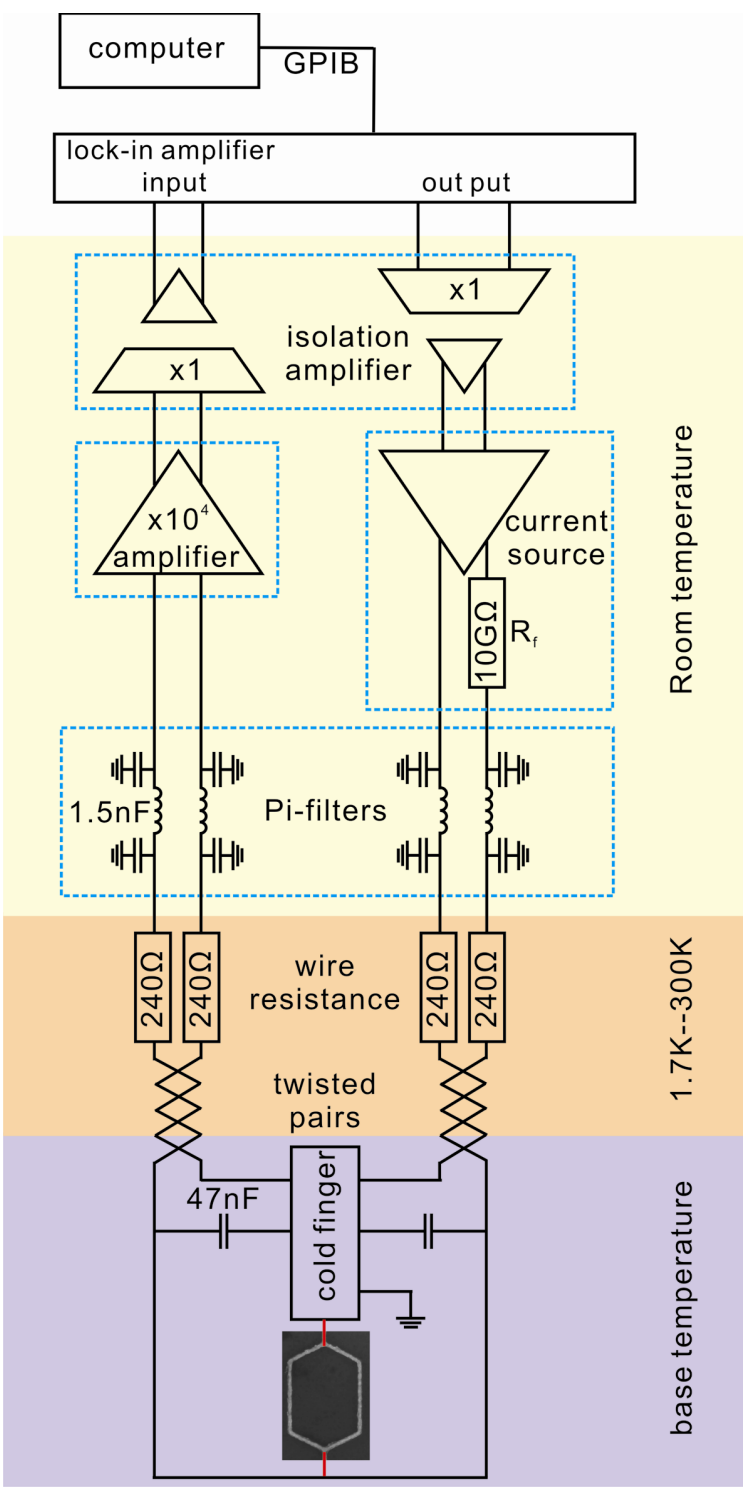

Figure 7.5: Circuit diagram of the measurement.

According to previous studies, the resistance oscillations originated from the $A B$ 
effect in metallic ring is about $0.05 \Omega$ around $10 \mathrm{mK}$ [11]. The excitation current was $200 \mathrm{nA}$, voltage signal is given by $\Delta V=I \Delta R=0.05 \Omega \times 200 \mathrm{nA}=10 \mathrm{nV}$ [11]. Thus, the noise and interference reduction in the electrical measurement setup is critical and somewhat an art. The absolute noise floor is given by the Johnson noise from the VI converter in the current source. The Johnson current noise is given by

$I=\sqrt{\frac{4 k T}{R_{\mathrm{f}}}}$,

where $k$ is Boltzmann's constant, $T$ is absolute temperature in $\mathrm{K}$ and $R_{\mathrm{f}}$ is the resistance of the feedback resistor in the current source. Since the source is at room temperature ( $300 \mathrm{~K}$ ), and $R_{\mathrm{f}}$ is $1 \times 10^{10} \Omega$, according to Eq. 7.1, the Johnson current noise is about $1.3 \mathrm{fA} / \sqrt{\mathrm{Hz}}$. To keep this noise floor, the noise and interference from other sources need to be minimized. The limitation of the noise level is found to be the $1 / f$ noise at the input of the voltage amplifier. $1 / f$ noise can result from a variety of effects, such as impurities in a conductive channel, generation and recombination noise in a transistor due to base current, and so on. Its origin is not well known. The $1 / f$ noise becomes dominant at low frequencies or in a DC measurement, since below a corner frequency voltage noise is proportional to $1 / f[12]$. Two different voltage amplifier modules were used (Model M2b and M2d, Quantum Transport, TU Delft, design by Ing. Raymond Schouten). These two JFET amplifiers have very low $1 / f$ noise at their inputs. For model $\mathrm{M} 2 \mathrm{~b}$, the noise floor is $2 \mathrm{nV} / \sqrt{\mathrm{Hz}}$. Model $\mathrm{M} 2 \mathrm{~b}$ has even lower noise floor $0.8 \mathrm{nV} / \sqrt{\mathrm{Hz}}$. To reduce the $1 / f$ noise and minimize the interference, a lock-in amplifier (Stanford Research Systems SR830) was used in the measurement. The frequency of the driving signal was kept below $200 \mathrm{~Hz}$ to realize a quasi-DC measurement.

Apart from the noise, a considerable current interference can be generated by vibrating measurement wires in the magnetic field due to electromagnetic induction. As shown in Fig. 7.5, the measurement configuration consists of multiple twisted pairs in loops. In this way, the random magnetic flux pickup due to the vibrating wires in magnetic field can be cancelled out. For each $A B$ ring, one twisted pair supplies the current and the other twisted pair probes the voltage. One wire in each twisted pair is connected to a terminal of the $A B$ ring, 
whereas the other wire is attached to the cold ground in order to avoid thermal voltages between ground levels. The other terminal of the ring is also attached to the cold ground, which helps cooling the electrons in the ring. This connection diagram provides the necessary configuration for the four point measurement.

Another common source of interference, with frequency predominantly at $50 \mathrm{~Hz}$ or multiples of this, originates from ground loops [12]. To prevent ground loop, the matrix rack, IV-VI rack and the main bath of the cryostat were connected to a single ground. To avoid multiple ground connections, rubber pumping lines and plastic connectors at the helium recovery lines were used. Also, the measurement electronics were isolated from other possible grounds by using a wooden measurement rack.

\subsection{Aharonov-Bohm measurements}

The thermal energy at a certain temperature $(T)$ gives the limit of the excitation current. As a rule of thumb, the electron energy should be lower than the thermal energy $\left(k_{B} T=22 \mu \mathrm{eV}\right.$ at $\left.250 \mathrm{mK}\right)$. For example, if the device has a resistance of $500 \Omega$, the maximum excitation current at $250 \mathrm{mK}$ is $44 \mathrm{nA}$ (Considering $I \times R \leq 22 \mu \mathrm{V} \approx 22 \mu \mathrm{eV}$ ).

$A B$ rings with two different diameters, $400 \mathrm{~nm}$ and $600 \mathrm{~nm}$ were chosen for the cryogenic measurements. These rings were bonded with $1 \%$ Silicon doped Al wires. The resistances of the rings were measured to check the states of the ring and the connections. The resistance was measured both at room temperature $\left(R_{\mathrm{T}}\right)$ and base temperature $250 \mathrm{mK}\left(R_{\text {base }}\right)$. For instance a $600 \mathrm{~nm}$ ring gives $R_{\mathrm{T}}=725.66 \pm 0.07 \Omega$ and $R_{\text {base }}=515.29 \pm 0.04 \Omega$. A $400 \mathrm{~nm}$ ring gives $R_{\mathrm{T}}=453.60 \pm 0.12 \Omega$ and $R_{\text {base }}=306.75 \pm 0.05 \Omega$. As the temperature is lowered, the resistance drops due to the reduced electron-phonon interaction.

After cooling down, the magnetoresistance (MR) of the sample was measured in a perpendicular magnetic field between $-7 \mathrm{~T}$ and $+7 \mathrm{~T}$. After this full range measurement, several small MR traces were chosen where the $A B$ oscillations were most clearly visible. These MR traces were again measured at various temperatures and excitation currents to investigate the temperature and 
excitation current dependence.

A $9^{\text {th }}$ order polynomial was fitted to the MR trace. Afterwards, the fit was subtracted from the MR trace. In this way, the contribution from the UCF is subtracted. A numerical fast Fourier transfer (FFT) was performed on the resulting $M R$ trace to extract the periodicity of the oscillation, which was compared to the theoretical period of the $A B$ oscillation (Appendix $C$ ). All the data sets presented in this chapter were processed using this procedure.

\subsubsection{Aharonov-Bohm oscillations}

A $500 \mathrm{mT}$ MR trace measured on a $600 \mathrm{~nm}$ ring $(\mathrm{H} 12)$ is shown in Fig. 7.6. The magnetic field was swept from $-5 \mathrm{~T}$ to $5 \mathrm{~T}$ with a step size of $2 \mathrm{mT}$; the excitation current was $30 \mathrm{nA}$ and the temperature was $262 \mathrm{mK}$. The MR traces of ring $\mathrm{H} 12$ before and after subtracting the polynomial fit in a $500 \mathrm{mT}$ MR trace are plotted in Fig. 7.6a and b.
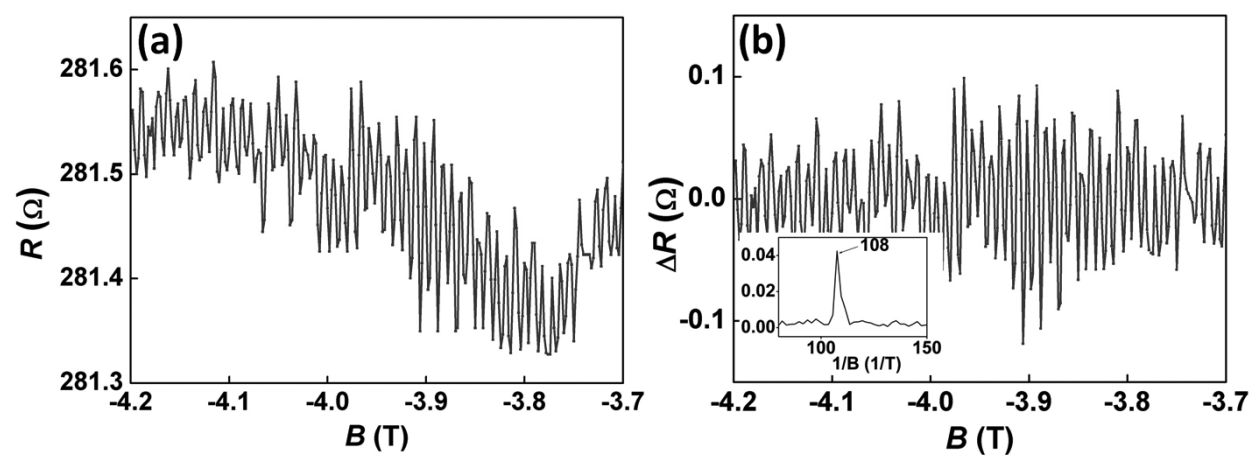

Figure 7.6: A $500 \mathrm{mT}$ MR trace of ring $\mathrm{H} 12$ before (a) and after (b) subtracting the polynomial fit. The insert in (b) gives its FFT result. A clear peak at position $108 \mathrm{~T}^{-1}$ was found. It corresponds to an oscillation period of $9.3 \mathrm{mT}(1 / 108)$.

Clear AB oscillations were observed. A clear peak at position $108 \mathrm{~T}^{-1}$ was found in the FFT. It corresponds to an oscillation period of $9.3 \mathrm{mT}(1 / 108)$ which agrees with the theoretical predicted period of $h / e(9.2 \mathrm{mT})$. This period is reproducible across different magnetic field intervals. In the FFT, only the $h / e$ peak was 
observed. The absence of $h / 2 e$ peak maybe due to the step size of the magnetic field sweep was too big, thus there are not enough points for the FFT. Based on further analysis, the $A B$ oscillation is most clearly visible in the magnetic field regime from $-1.7 \mathrm{~T}$ to $-1.2 \mathrm{~T}$. This regime was chosen for a couple of other experiments (for example: temperature and excitation dependence) for this particular ring.

$A B$ oscillations were also observed for a $400 \mathrm{~nm}$ ring (D12). Reproducible $h / e$ oscillations were found with period $20.8 \mathrm{mT}$, consistent with the predicted value (20.7 mT). The AB period is also reproducible across different ranges. On this 400 $\mathrm{nm}$ ring, two $200 \mathrm{mT}$ (5.5 T to 5.3 T) MR traces were measured in opposite sweep directions with a step size of $1 \mathrm{mT}$. As shown in Fig. 7.7, the two MR traces coincided with each other independent of the sweeping direction. It confirms the oscillation in the MR is originated by the AB effect [13].

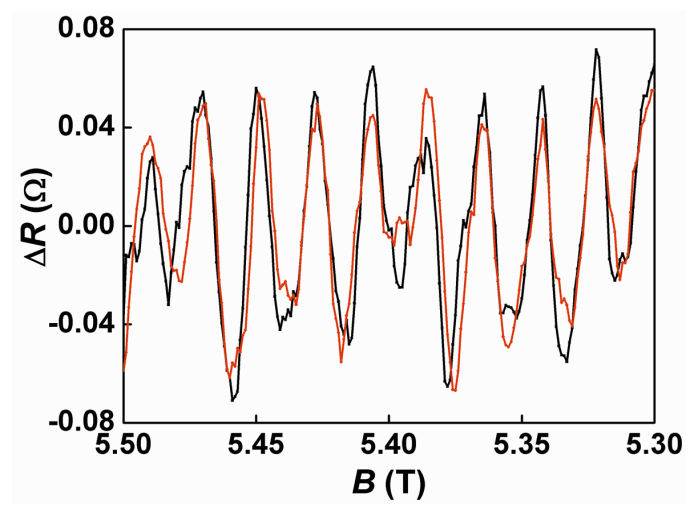

Figure 7.7: A $200 \mathrm{mT}$ long MR trace of ring D12 (5-point smoothing). The sweeping direction of magnetic field is $5.5 \mathrm{~T}$ to $5 \mathrm{~T}$ (black) and $5 \mathrm{~T}$ to $5.5 \mathrm{~T}$ (red).

\subsubsection{Temperature dependence of $\mathrm{AB}$ oscillations}

The $400 \mathrm{mT}$ long (-1.2 $\mathrm{T}$ to $-1.6 \mathrm{~T}) \mathrm{MR}$ traces from a $600 \mathrm{~nm}$ ring $(\mathrm{H} 12)$ were taken at different temperature with $30 \mathrm{nA}$ excitation current and $0.4 \mathrm{mT}$ magnetic field steps (Fig. 7.8). 


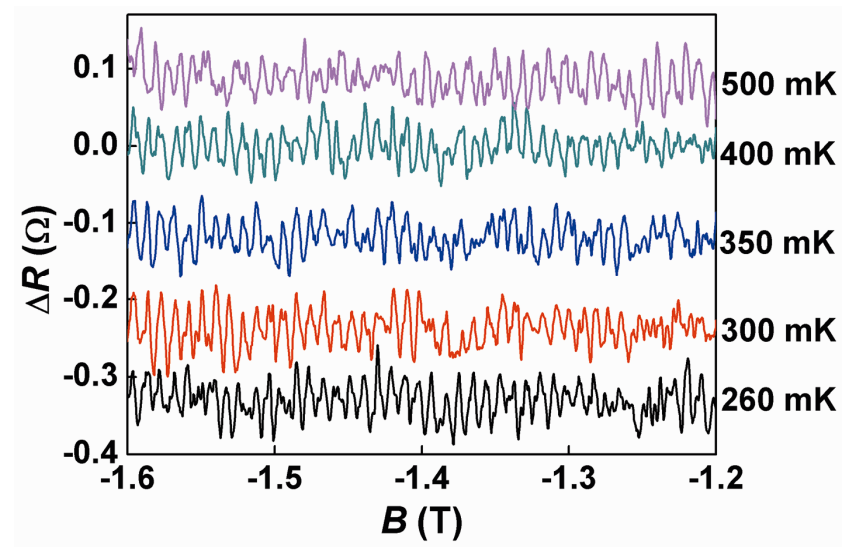

Figure 7.8: The $400 \mathrm{mT}$ long MR traces of ring $\mathrm{H} 12$ taken at different temperatures (smoothed for 5points).

$A B$ oscillations are clearly observed at all temperatures (from $260 \mathrm{mK}$ to $350 \mathrm{mK}$ ). The $h / e$ oscillation period obtained from all these measurements were consistent (9.3 $\mathrm{mT})$. Increase in temperature slightly decreases the oscillation quality, likely due to a reduction in coherence length. However, even at $350 \mathrm{mK}, l_{\varphi}>L / 2$ is still satisfied [8-9]. As a result, temperature has no clear effect on the $A B$ oscillations in this temperature regime.

\subsubsection{Excitation Current Dependence of AB oscillations}

The excitation current dependence of the $A B$ oscillations was studied for a 600 $\mathrm{nm}$ ring (H12). The excitation current was varied from $20 \mathrm{nA}$ till $35 \mathrm{nA}$ in the field regime $-1.7 \mathrm{~T}$ to $-1.3 \mathrm{~T}$ with a step size of $0.4 \mathrm{mT}$. The temperature was $\sim 260 \mathrm{mK}$. The $400 \mathrm{mT}$ MR traces at different excitation current are plotted in Fig. 7.9. The h/e oscillations with a period of $9.3 \mathrm{mT}$ are clearly observed at different excitation currents. The quality of the oscillation improves dramatically at larger excitation current (30 and $35 \mathrm{nA}$ ). In similar measurement configurations, the voltage noise floor was determined $(\approx 1 \mathrm{nV}$ peak to peak). Increasing the excitation leads to a better signal to noise ratio (assuming an average resistance oscillation $\approx 0.06 \Omega$, the signal to noise ratio for $I=20 \mathrm{nA}$ is $1.2 ; l=30 \mathrm{nA} 1.8$ and 
$I=35 \mathrm{nA}, 2.1$ ). As a result, the oscillation became more evident at higher currents.

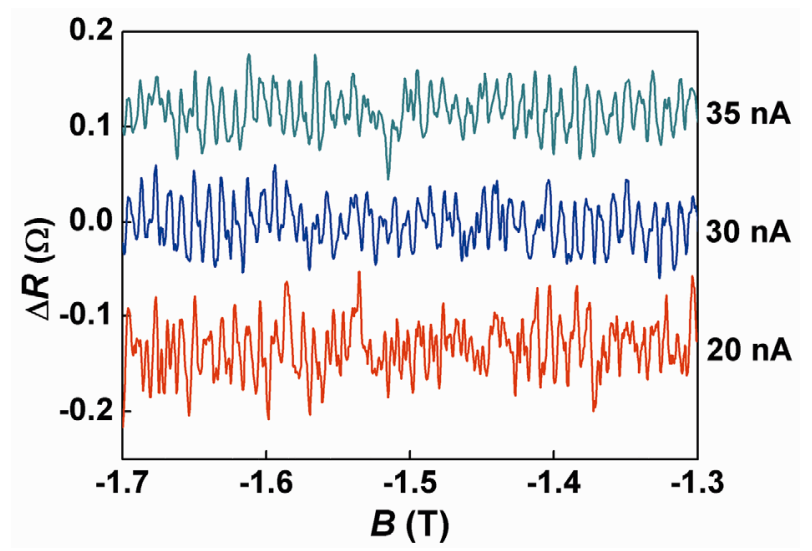

Figure 7.9: The $400 \mathrm{mT}$ long MR traces of ring $\mathrm{H} 12$ taken at different excitation currents (5-point smoothing).

\subsection{Conclusion}

$A B$ oscillations were observed for rings with different sizes. The expected and measured $A B$ periods are in good agreement. Within the temperature and excitation current ranges, the $A B$ periods were robust. The molecular insertion via NPs bridging will be tested in near future. Then with an optimized balance junction, the first coherence transport through organic molecule could be realized.

\section{References}

[1] R. A. Webb, S. Washburn, C. P. Umbach, R. B. Laibowitz, Physical Review Letters 54 (1985) 2696.

[2] V. M. Galitski, M. G. Vavilov, L. I. Glazman, Physical Review Letters 94 (2005) 096602.

[3] L. C. Mur, C. J. P. M. Harmans, W. G. Wiel van der, New Journal of Physics 10 (2008) 073031. 
[4] I. Amlani, A. M. Rawlett, L. A. Nagahara, R. K. Tsui, Applied Physics Letters 80 (2002) 2761.

[5] R. J. Barsotti, M. D. Vahey, R. Wartena, Y.-M. Chiang, J. Voldman, F. Stellacci, Small 3 (2007) 488.

[6] C. Chu, J.-S. Na, G. N. Parsons, Journal of the American Chemical Society 129 (2007) 2287.

[7] K. Aihara, M. Yamamoto, K. Iwadate, T. Mizutani, Japanese Journal of Applied Physics, Part 1: Regular Papers and Short Notes and Review Papers 30 (1991) 1627.

[8] G. Dumpich, A. Carl, Physical Review B 43 (1991) 12074.

[9] A. Trionfi, S. Lee, D. Natelson, Applied Physics Letters 89 (2006) 262104.

[10] Heliox operator handbook Oxford Instruments

[11] R. A. Webb, S. Washburn, C. P. Umbach, R. B. Laibowitz, Physical Review Letters 54 (1985) 4.

[12] Low level measurements handbook 6th edition Keithley Instruments, Inc.

[13] V. F. Gantmakher, Electrons and Disorder in Solids Oxford University Press (2005) 
Page | 104 


\section{Tunable Molecular Spin Doping of a Metal}

The mutual interaction of localized magnetic moments and their interplay with itinerant conduction electrons in a solid is a key fundamental topic in solid-state physics. These mechanisms form the very basis of magnetic ordering and related many-body phenomena, such as the Kondo effect [1], Ruderman-Kittel-Kasuya-Yoshida (RKKY) interaction [2], and carrier-induced ferromagnetism in diluted magnetic semiconductors [3]. Crucial parameters determining the strength and the relative importance of these spin phenomena, are the magnitude and sign of the exchange interaction, and the mean distance between the localized magnetic moments. To enable systematic study of a given magnetic impurity-host system, straightforward tuning of the mean distance between the impurities is highly desirable, as this sets its physical properties. Here, a novel, facile molecular fabrication method for inserting isolated localized magnetic moments in a gold film with tunable density is presented. Kondo and weak localization measurements demonstrate that the magnetic impurity concentration can be systematically varied up to $680 \mathrm{ppm}$ concentration without any sign of inter-impurity interaction, or undesired clustering often suffered from in alternative methods. These results indicate that even for inter-impurity distances far below the Kondo length scale no coupling of magnetic impurities in gold occurs.

This chapter has been submitted to Nature Nanotechnology as T. Gang, M. Deniz Yilmaz, D. Ataç, S.K. Bose, E. Strambini, A.H. Velders, M.P. de Jong, J. Huskens and W.G. van der Wiel. 


\subsection{Introduction}

The inclusion of even a small amount of magnetic impurities in a metal can have drastic effects on the physical properties, such as the electrical resisitivity and magnetic susceptibility. A well-known example is the anomalous electrical resistivity minimum at low temperature, referred to as the Kondo effect [1]. Another example is the reduction of the carrier phase coherence time due to enhanced spin scattering introduced by the impurity magnetic moments [4]. For systematic experimental study of such phenomena, good control of the magnetic impurity concentration is indispensable. The most common method for varying the amount of magnetic impurities is by alloying a non-magnetic and magnetic metal in different ratios. The preparation of such alloys is far from trivial though, and each desired impurity concentration needs to be prepared separately. It requires delicate procedures such as melting, swaging, etching, annealing, and quenching to ensure uniform mixing and avoid element segregation [5]. Moreover, impurity concentrations are limited up to about 100 ppm, as for higher values segregation or clustering occurs. In a few other studies, magnetic atoms were deposited on a host metal ( $\mathrm{Au}$ ) in ultra-high vacuum and at cryogenic temperatures to prevent agglomeration [4]. However, also here impurity agglomeration is very hard to exclude at a few 100 ppm level.

In this chapter, a novel, facile molecular fabrication method for magnetic doping up to high concentrations of isolated impurities is presented. The power and versatility offered by molecular bottom-up assembly are more and more exploited in the fabrication of electronic devices. As the critical dimensions approach the molecular scale, the application of molecules as building blocks or scaffolds for electronic components becomes increasingly attractive. Of particular interest are (self-assembled) molecular monolayers, which, owing to their self-limited growth, offer large-area 2D systems that can be engineered at the molecular scale. Molecular monolayers have been applied for tuning metal work functions [6], for surface transfer doping of semiconductors [7], for self-assembled-monolayer field-effect transistors (SAMFETs) [8], for the modification of the electronic properties of grapheme [9], and for uniform [10] and patterned [11] doping of silicon with donor atoms. Magnetic doping from a molecular monolayer, however, has not been reported yet. 
This method consists of inserting isolated localized magnetic impurities in a gold film from a monolayer of metal-ligand complexes containing a metal ion with an unpaired spin ("spin donor"). By mixing in molecules with the same organic ligands, but with a zero-spin metal core ion, the concentration of magnetic moments can be controlled straightforwardly. Low-temperature electron transport measurements exhibiting the Kondo effect and weak (anti-)localization, confirm the systematic variation of the magnetic impurity density up to $680 \mathrm{ppm}$ [12]. No sign of impurity coupling (nor clustering) was observed, which opens up new ways to investigate the physics of high-density magnetic impurity systems.

\subsection{The metal terpyridine complex}

The inset of Fig. 8.1a shows the metal terpyridine complex used in this study (see Methods for details on the synthesis). The metal core is chosen to be either a $\mathrm{Co}^{2+}$ or $\mathrm{Zn}^{2+}$ ion, and is coordinated in a distorted octahedral manner by two terpyridine ligands. To one of the ligands a thiol (-SH) group is attached, which allows to form a monolayer on an Au surface. Whereas $\mathrm{Zn}^{2+}$ is spinless, $\mathrm{Co}^{2+}$ does carry a spin. At low temperatures relevant for this work $(2-80 \mathrm{~K}), \mathrm{Co}^{2+}$ is known to be in the low-spin ground state with $S=1 / 2$ [13]. By cyclic voltammetry (see Methods), it was confirmed that $\mathrm{Co}^{2+}$ in the bis-terpyridine complex can be oxidized to zero-spin $\mathrm{Co}^{3+}$ at relatively low energy $(\sim 0.25 \mathrm{eV})$, a property that allows for Kondo physics at low temperature [14].

\subsection{Sample fabrication}

Monolayers were formed from $0.1 \mathrm{mM}$ mixed complex solutions having different ratios of $\mathrm{Co}^{2+}$ and $\mathrm{Zn}^{2+}$ complexes $(0,25,50,67$ and 100\% Co), on separate $\mathrm{Si} / \mathrm{SiO}_{2} / \mathrm{Au}$ substrates. The $2.5 \mathrm{~nm} \mathrm{Au}$ films (99.99\% Au source) were sputter deposited (without adhesion layer) in a single run (see Methods for a detailed description of the fabrication process). The metal deposition process was optimized for getting sufficiently disordered films, suitable for weak localization measurements (described below). As the molecular complexes are identical (in outer ligand structure and overall charge), except for their core metal ion (i.e. 
either $\mathrm{Co}^{2+}$ or $\mathrm{Zn}^{2+}$ ), the same adsorption rate is expected for both species. Therefore a homogeneously mixed monolayer on top of the Au film, reflecting the Co: $\mathrm{Zn}$ ratio of the mixed donor solution is anticipated. The low-temperature electron transport measurements (see below) confirm that this is indeed the case. From electrochemical characterization (see Methods), a molecular coverage of $5 \times 10^{-11} \mathrm{~mol} / \mathrm{cm}^{2}$ was estimated, corresponding to $\sim 1$ molecule per 3 $\mathrm{nm}^{2}$. After monolayer formation, the samples were covered with a top Au layer of the same thickness as the bottom layer [15]. Based on the measured molecular coverage and the total thickness of the Au layer, a maximum realizable magnetic impurity concentration on the order of $10^{3} \mathrm{ppm}$ is expected.

a

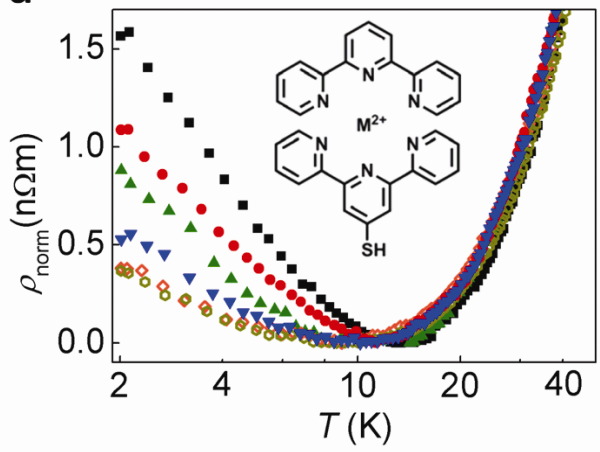

b

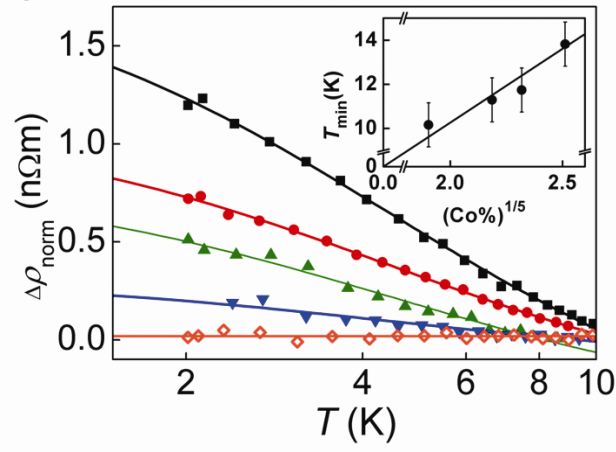

Figure 8.1: Kondo effect. a, Temperature dependence of the normalized resistivity,

$\rho_{\text {norm }}(T)=\frac{\rho(T)-\rho_{\min }}{\rho(150 \mathrm{~K})-\rho_{\min }} \times \rho_{\mathrm{Au}}(150 \mathrm{~K})$ of a (99.99\%) pure Au film (green hexagons), and the same $\mathrm{Au}$ film with inserted molecular monolayers made from mixed Co complex[x\%]/Zn complex[(100-x)\%] solutions with $\mathrm{x}=0$ (orange diamonds), 25 (blue down triangles), 50 (green up triangles), 67 (red dots), and 100 (black squares). Inset: structure of metal terpyridine complex, with " $M$ " being either Co or Zn. b, Data of (a) with the bare Au film curve subtracted. The solid curves are fits to Eq. (8.1). Inset: $T_{\min }$ values of the resistivity minima $\rho_{\text {min }}$, showing $(\mathrm{Co} \%)^{1 / 5}$ dependence.

\subsection{Temperature dependent resistivity measurements}

Electron transport measurements were performed on Au films molecularly spin doped from donor solutions with different Co complex: $\mathrm{Zn}$ complex ratios, as well as on a reference sample immersed in a solution without metal complexes, but 
fabricated exactly the same otherwise. Figure 8.1a shows the temperature dependence of the film resistivity for different Co concentrations. Although the bottom and top Au layers have been deposited in the same deposition runs for all samples, there are slight sample-to-sample resistivity variations. To make direct comparison possible, the resistivity values have been normalized according to $\rho_{\text {norm }}(T)=\frac{\rho(T)-\rho_{\min }}{\rho(150 \mathrm{~K})-\rho_{\min }} \times \rho_{\mathrm{Au}}(150 \mathrm{~K})$ with $\rho_{\min }$ the minimum resistivity value of each curve, and $\rho_{\mathrm{Au}}(150 \mathrm{~K})$ the resistivity of the bare Au film at $150 \mathrm{~K}$. For the highest temperatures, the resistivity of all films has the same $T^{5}$ dependence, characteristic for phonon scattering. All curves, however, show a resistivity minimum $\rho_{\min }$ at $T_{\min }$ between $10 \mathrm{~K}$ and $20 \mathrm{~K}$.

The low-temperature resistivity shows a logarithmic upturn (Fig. 8.1a), which increases with the amount of Co in the donor solution. The resistivity minimum and logarithmic upturn are indicative of the Kondo effect, which arises from the interaction of a localized magnetic impurity with the spin of itinerant conduction electrons in the host metal [1]. Coherent superposition of (higher-order) spin-flip scattering eventually leads to the complete screening of the localized moment below a critical temperature, referred to as the Kondo temperature, $T_{\mathrm{K}}$. A many-body singlet state is formed by the impurity and a "cloud" of conduction electrons. As the scattering cross section of this Kondo cloud is larger than that of the original impurity, the resistivity increases in case of bulk diluted impurity systems. The Kondo effect is in essence a single-impurity phenomenon. In metals, however, the resistivity upturn results from the contribution of many uncorrelated magnetic impurities. More recently, the Kondo effect has also been studied at the single-impurity level, using scanning tunneling microscopy of single magnetic impurities formed by an adatom or molecule on a metal substrate [16-17], or using electron transport through semiconductor quantum dots [18] or single molecules [14]. In such systems, it was shown that the impurity can indeed be completely screened [19].

The growing Kondo resistivity upturn with increasing Co donor solution concentration as shown in Fig. 8.1a, demonstrates that the metal film is effectively doped with localized magnetic impurities from the molecular monolayer. In order to quantitatively compare the resistivity data with Kondo 
theory, only the incremental resistivity $\Delta \rho_{\text {norm }}(T)$ obtained by subtracting the $\rho_{\text {norm }}(T)$ curve of the bare Au film from all other $\rho_{\text {norm }}(T)$ curves is considered. As seen in Fig. 8.1a, the bare Au film data also show a small low-temperature resistivity upturn. This is attributed to magnetic impurities (most likely Fe) in the $99.99 \%$ pure Au source material, and to weak localization and electron-electron interactions, which can also contribute to a temperature-dependent part of the resistivity [20-21]. As these contributions should be the same for all samples studied, $\Delta \rho_{\text {norm }}(T)$ is expected to only reflect the effect of molecular spin doping.

Figure $8.1 \mathrm{~b}$ shows the thus obtained $\Delta \rho(T)$ curves, fitted to the Hamann expression [22]

$\Delta \rho=\frac{2 \pi c}{n e^{2} k_{F}}\left\{1-\ln \left(\frac{T}{T_{\mathrm{K}}}\right) \times\left[\ln ^{2}\left(\frac{T}{T_{\mathrm{K}}}\right)+S(S+1) \pi^{2}\right]^{-1 / 2}\right\}$,

where $c$ is the magnetic impurity concentration, $n$ the electron density and $k_{F}$ the Fermi wave vector of the host metal, and $S$ a value reflecting the total impurity spin. It was found $T_{\mathrm{K}}=4.2 \mathrm{~K}$ and $S=0.27$ for all curves, and impurity concentrations of $0,120,325,400$, and $680 \mathrm{ppm}$, for Co complex concentrations of $0 \%, 25 \%, 50 \%, 67 \%$ and $100 \%$, respectively. The inset of Fig. $8.1 \mathrm{~b}$ shows that $T_{\min }$ scales with the Co concentration as $(\mathrm{Co} \%)^{1 / 5}$, as expected for a Kondo system of non-interacting impurities [23].

The impurity concentrations derived from the Hamann fits, are found to be directly proportional to the Co complex concentration in the donor solution, see Fig. 8.2. This not only demonstrates that by mixing the Co and $\mathrm{Zn}$ complexes in solution the magnetic impurity concentration can be effectively controlled, but also confirms that the Co complex: $\mathrm{Zn}$ complex ratio in the monolayer is the same as the ratio in the donor solution. Importantly, no sign of saturation of the impurity concentration is observed, implying that no clustering of magnetic dopant atoms occurs even for the highest concentrations studied. The $680 \mathrm{ppm}$ concentration found for the $100 \%$ Co sample corresponds reasonably well with the $\sim 10^{3} \mathrm{ppm}$ concentration expected from the monolayer coverage (see Methods). 


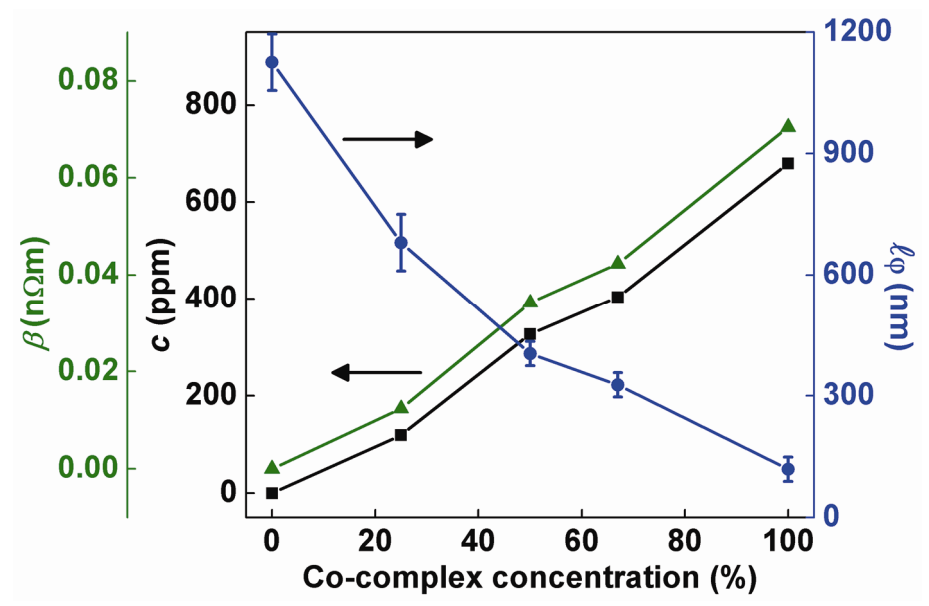

Figure 8.2: Tunable molecular spin doping. Magnetic impurity concentration, $c$, (black squares) derived from the fits to Eq. (8.1) in Fig. 8.1b, $\beta$ value (green triangles) of Eq. (8.2) obtained from the slope at $T_{\mathrm{K}}=4.2 \mathrm{~K}$ in Fig. $8.1 \mathrm{~b}$, and phase coherence length $l_{\phi}(2 \mathrm{~K})$ (blue dots) derived from the fits to Eq. (8.4) in Fig. 8.3b, versus the Co complex concentration in the donor solution.

In the Kondo regime, i.e. in the case of non-interacting magnetic impurities, and at temperatures above and around $T_{K}$, the resistivity increase is well described by

$\Delta \rho=-\beta \ln (T)$,

Where $\beta$ is a positive coefficient with a magnitude that depends on the host material and is proportional to the magnetic impurity concentration [21]. The $\beta$ values are obtained from the data in Fig. 8.1b, by taking the slope at $T_{\mathrm{K}}=4.2 \mathrm{~K}$, and are also plotted in Fig. 8.2. $\beta$ is indeed directly proportional to c (and hence to the Co complex concentration). Therefore, up to the highest impurity concentration of $680 \mathrm{ppm}$, the samples are still in the Kondo regime. This is a significant finding, as so far, pure Kondo behaviour has never been reported for such high impurity concentrations in Au. In other studies on Au/impurity systems [24-25], for similar or lower concentrations, the onset of impurity-impurity (RKKY) interaction was sometimes put forward to explain deviations from the behaviour observed at the lowest impurity levels. The present work, however, suggests the 
absence of inter-impurity interactions up to very high concentrations.

This molecular spin doping scheme thus allows the creation of a system of non-interacting magnetic impurities at a doping level very hard to achieve with alternative methods. A concentration of $680 \mathrm{ppm}$ corresponds to a mean inter-impurity distance of $\sim 3 \mathrm{~nm}$ (see Methods). This value is compared to the Kondo length scale, $l_{K}$, which has been proposed as a measure for the extension of the Kondo cloud [21]:

$l_{K} \sim \frac{\hbar v_{F}}{2 \pi k_{B} T_{K}}$,

where $\hbar$ is the reduced Planck's constant, $v_{F}$ the Fermi velocity of the conduction electrons, and $k_{B}$ the Boltzmann constant. With $T_{K}=4.2 \mathrm{~K}, l_{K} \sim$ $400 \mathrm{~nm}$ was found, two orders of magnitude larger than the mean inter-impurity distance derived above. It indicates that the Kondo length (at least in this case) is not a good indicator for the onset of impurity coupling, as pure Kondo behavior were observed continuously for mean inter-impurity distances well below $l_{K}$. A similar conclusion was reached in earlier studies on $\mathrm{Au}(\mathrm{Fe})$ [21], although for much lower impurity concentrations than described here.

\subsection{Magnetic field dependent resistivity measurements}

In order to verify the molecular spin doping method in an independent way, low-temperature magnetoresistance (MR) measurements was performed. Figure 8.3a shows the normalized MR values $\frac{\left[\Delta R_{\square}(B)-\Delta R_{\square}(0)\right]}{R_{\square}(0) R_{\square}(B)} \equiv-\Delta \sigma$ for different Co complex concentrations at $2 \mathrm{~K}[26]$. A minimum at $B=0$ is found for all curves, attributed to weak anti-localization (WAL). Weak localization (WL) and WAL are quantum corrections to the resistivity due to coherent back scattering in disordered systems [4]. In case of weak spin-orbit coupling, the effect leads to an enhanced probability for the carriers to return to the point of origin, i.e. WL, and therefore to an increased resistivity. However, in solids with large spin-orbit coupling, as for $\mathrm{Au}$ in this case, WAL occurs, which gives a negative quantum contribution to the resistivity. WL and WAL are suppressed by a (perpendicular) magnetic field, lifting time-reversal symmetry, which provides a way to derive the 
carrier phase coherence length, $l_{\phi}$ [4]. The normalized MR curves of Fig. 8.3a show clear WAL minima, with a width that strongly increases with Co complex concentration.
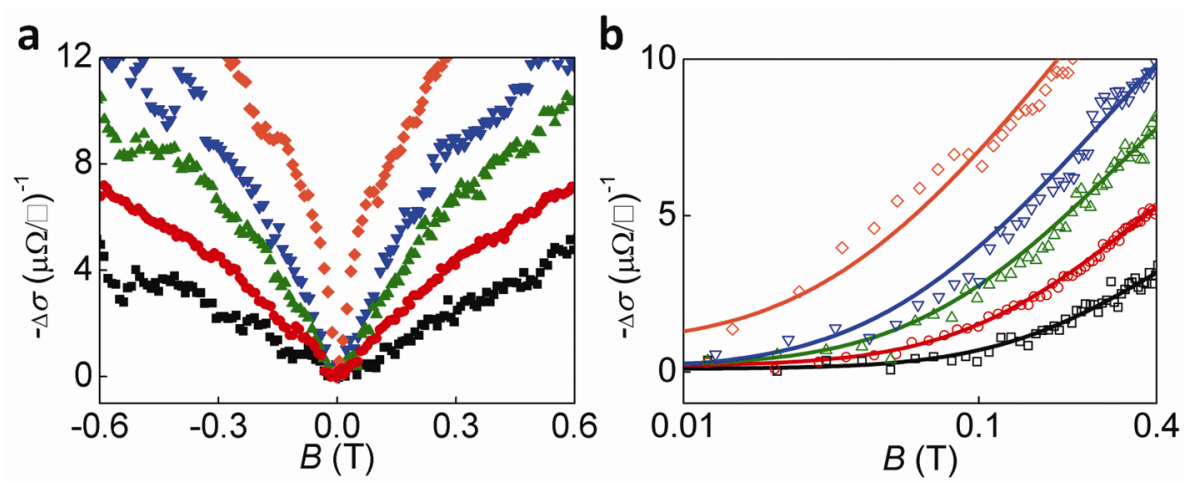

Figure 8.3: Magnetoresistance. a, Magnetoresistance $\frac{\left[\Delta R_{\square}(B)-\Delta R_{\square}(0)\right]}{R_{\square}(0) R_{\square}(B)} \equiv-\Delta \sigma$ at $2 \mathrm{~K}$ for the same $\mathrm{Au}$ film with inserted molecular monolayers made from mixed Co complex[x\%]/Zn complex[(100-x)\%] solutions with $x=0$ (orange diamonds), $x=25$ (blue down triangles), 50 (green up triangles), 67 (red dots), and 100 (black squares). b, Data of (a) fitted to weak localization theory, Eq. (8.4), with $B_{\mathrm{e}}=57 \mathrm{~T}$ and $B_{\mathrm{so}}=0.13 \mathrm{~T}$.

In Fig. 8.3b the low-field data are fitted with the theory of Hikami, Larkin and Nagaoka[27]

$$
\Delta \sigma(B)=-\frac{e^{2}}{2 \pi^{2} \hbar \hbar}\left[f\left(\frac{B_{1}}{B}\right)-\frac{3}{2} f\left(\frac{B_{2}}{B}\right)+\frac{1}{2} f\left(\frac{B_{\phi_{\emptyset}}}{B}\right)\right]
$$

where $f(x)=\psi\left(x+\frac{1}{2}\right)-\ln (x)$ with $\psi(x)$ the digamma function, $B_{1}, B_{2}$ and $B_{\phi}$ are characteristic magnetic fields defined by $B_{1}=B_{e}+B_{\text {s.o. }}+B_{S}, B_{2}=B_{i}+$ $\frac{4}{3} B_{\text {s.o. }}+\frac{2}{3} B_{S}$ and $B_{\phi}=B_{i}+2 B_{s}$, referring to contributions from elastic (e), inelastic (i), spin-orbit (s.o.) and magnetic (s) scattering. At low magnetic fields, $B_{1}$ is dominated by $B_{\text {s.o. }}$ which is independent of magnetic field. The same holds for $B_{2}$, leaving $B_{\phi}$ as the parameter determining the shape of the WAL curves. From $B_{\phi}$ the phase coherence length can be derived via $l_{\phi}(2 \mathrm{~K})=$ $\sqrt{\frac{\hbar}{4 e B_{\phi}(2 K)}}$ for the different Co complex concentrations, which are also plotted in Fig. 8.2. The phase coherence length monotonically decreases from $1.1 \mu \mathrm{m}$ to 
$150 \mathrm{~nm}$ with increasing Co complex concentration. As the inelastic scattering contribution is expected to be the same for all samples, the decrease of $l_{\phi}$ with increasing Co complex concentration can be fully attributed to enhanced (phase-breaking) spin scattering of the introduced magnetic impurities. These results thus provide independent evidence that the magnetic impurity concentration can be tuned effectively via this molecular spin doping scheme.

\subsection{Conclusion}

A conceptually novel and very easy approach to dope metal films with a tunable amount of magnetic impurities from a molecular monolayer has been demonstrated. Kondo and magnetoresistance measurements demonstrate that the impurity concentration in the metal is directly proportional to the molecular spin dopant concentration in solution. Although up to now it was widely believed that inter-impurity coupling sets in at $\sim 100 \mathrm{ppm}$ levels, this molecularly doped impurity systems show pure Kondo behaviour down to $2 \mathrm{~K}$ at impurity levels as high as $680 \mathrm{ppm}$. This technique can be a powerful way to reach unprecedented impurity concentrations, while avoiding undesired aggregation or segregation. This should pave the way for further study of spin phenomena that lie at the very heart of solid-state physics, such as the Kondo effect, RKKY interaction, spin glasses, and magnetic phase transitions.

\subsection{Methods}

\section{Sample fabrication and electron transport measurement:}

The two metal terpyridine complexes $\mathrm{Co}(\mathrm{Tpy})(\mathrm{Tpy}-\mathrm{SH})$ and $\mathrm{Zn}(\mathrm{Tpy})(\mathrm{Tpy}-\mathrm{SH})$ used in this study, were synthesized according to literature procedures [28-29]. Freshly sputtered $2.5 \mathrm{~nm}$ gold thin films on $\mathrm{Si} / \mathrm{SiO}_{2}$ substrates $(2.2 \mathrm{~mm} \times 6.8 \mathrm{~mm}$ ) were used for monolayer formation. The monolayers were formed by dipping of a substrate in a $0.1 \mathrm{mM}$ solution of a mixture of the metal complexes (at Co: $\mathrm{Zn}$ ratios 1:0, 2:1, 1:1, 1:3 and 0:1) in acetonitrile overnight at room temperature. The substrates were rinsed with acetonitrile afterwards to remove any excess of 
compounds, and subsequently dried in a nitrogen stream. The gold capping layer was sputtered immediately after the monolayer formation. The total thickness of the gold film was $5 \mathrm{~nm}$. Sample resistance was measured in a standard four-terminal configuration with an excitation current between 0.5 and $1.5 \mu \mathrm{A}$.

\section{Electrochemistry:}

Electrochemical measurements were performed with an AUTOLAB PGSTAT10 in a custom-built three-electrode setup with bare gold substrates as the working electrode, an $\mathrm{Ag} / \mathrm{AgCl}$ reference electrode, and a platinum wire as counter electrode.

To determine the coverage of a monolayer of $100 \%$ Co complex on the $\mathrm{Au}$ surface, cyclic voltammetry measurements were performed, which are shown in Fig. 8.4a. Measurements were done with an $\mathrm{Au}$ electrode covered with a monolayer of the Co complex in a $0.1 \mathrm{mM}$ tetra-n-butylammonium hexafluorophosphate acetonitrile solution. A peak was observed around $0.375 \mathrm{~V}$, which was attributed to the redox activity of the $\mathrm{Co}^{2+}$ ion. Integration of the I-V curves gives the total charge $Q$, from which the surface coverage $\Gamma$ of the monolayer can be determined by $\Gamma=\frac{Q}{n F A}$, where $n$ is the number of electrons per mol of reaction, $F$ the Faraday constant and $A$ the surface area. By taking I-V curves for different scan rates, $Q$ could be determined as a function of the scan rate, as shown in Fig. 8.4b. The independence of $Q$ on the scan rate shows that it is a surface-confined process, as is expected for a monolayer [30]. $\Gamma=5.0 \times$ $10^{-11} \mathrm{~mol} \mathrm{~cm}^{-2}$ was found.
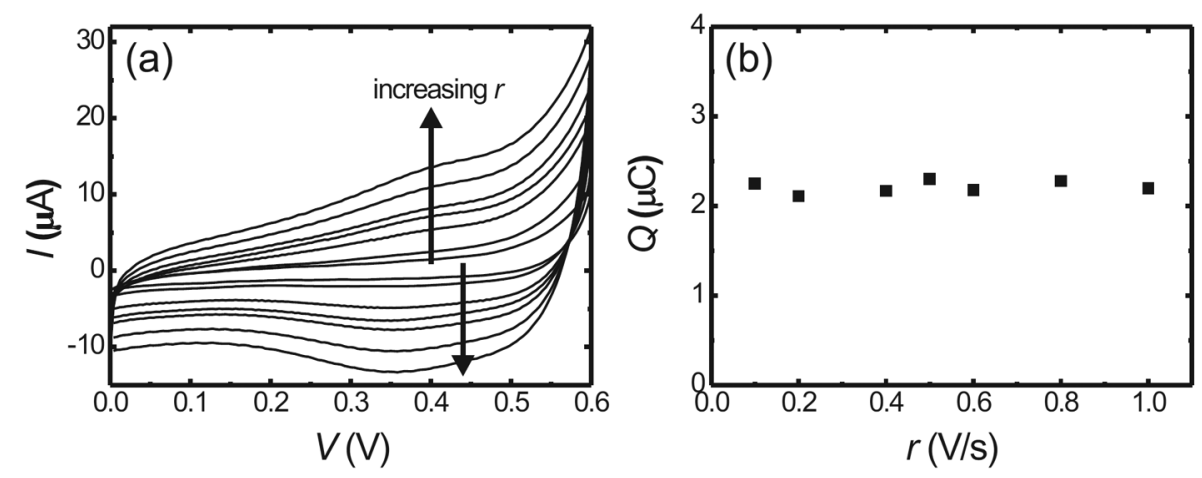

Figure 8.4: Cyclic voltammetry measurements on a monolayer of the Co complex. (a) 
Current $I$ vs. voltage $V$ for different scan rates $r$ (the direction of the arrows denote increasing $r$ ) and (b) charge $Q$ vs. scan rate, obtained from the curves in (a).

\section{Calculations for maximum impurity concentration:}

The maximum amount of Co complex $\left(n_{\mathrm{Co}}\right)$ on the $6.8 \times 2.2 \mathrm{~mm}^{2}$ $\mathrm{Si} / 300 \mathrm{nmSiO}_{2} / 5 \mathrm{nmAu}$ substrate surface was obtained from $n_{C o}=\Gamma S N_{A}$, where $S$ is the surface area of the substrate and $N_{A}$ is the Avogadro constant. The amount of gold atoms $\left(n_{\mathrm{Au}}\right)$ in $5 \mathrm{~nm}$ Au layer was derived by $n_{\mathrm{Au}}=\frac{\rho V}{M} N_{A}$, where $\rho$ is the density of Au at room temperature, $V$ is the volume of the gold film and $M$ is the molar mass of Au. The maximum impurity concentration in ppm is given by $c=1 \times 10^{6} \frac{n_{C o}}{n_{A u}} \approx 10^{3} \mathrm{ppm}$.

\section{Estimate for mean distance between spin impurities:}

From above, it is clear that the Co concentration ' $c$ ' (in ppm) is proportional to the Co complex molecular coverage ' $\Gamma$ ' (in $\mathrm{mol} / \mathrm{cm}^{2}$ ). So the molecular coverage corresponding to $c=680 \mathrm{ppm}$ can be estimated by $\Gamma_{680 \mathrm{ppm}} \approx \frac{680}{1000} \Gamma_{1000 \mathrm{ppm}}=3.4 \times 10^{-11} \mathrm{~mol} \mathrm{~cm}^{-2}$. This means one molecule per $5 \mathrm{~nm}^{2}$. Assuming all the spin impurities are distributed in a 2D plane with close packing, the mean inter-impurity distance is approximately $\sqrt{2 \times 5 \mathrm{~nm}^{2}} \approx$ $3 \mathrm{~nm}$. Assuming homogenous (3D) distribution through the whole film, a very similar number was found as the film thickness is of same order as the inter-impurity distance.

\section{Top Au deposition:}

After monolayer formation, the samples were covered with a top Au layer of same thickness as the bottom layer. It was found that the deposition of the top layer is essential for getting sufficient interaction between the spin dopants and the conduction electrons in the Au film. The organic ligands are expected to be (partly) destroyed in this process. Figure 8.5 shows temperature-dependent normalized resistivity plots of the $0 \%$ and $100 \%$ Co complex monolayer formed on top of a $5 \mathrm{~nm}$ Au surface. The $100 \%$ Co complex sample had a slightly higher resistivity upturn than the $0 \%$ Co complex sample at $\mathrm{T}<4 \mathrm{~K}$, much weaker compared to the $100 \%$ Co-complex sample with the top Au. 


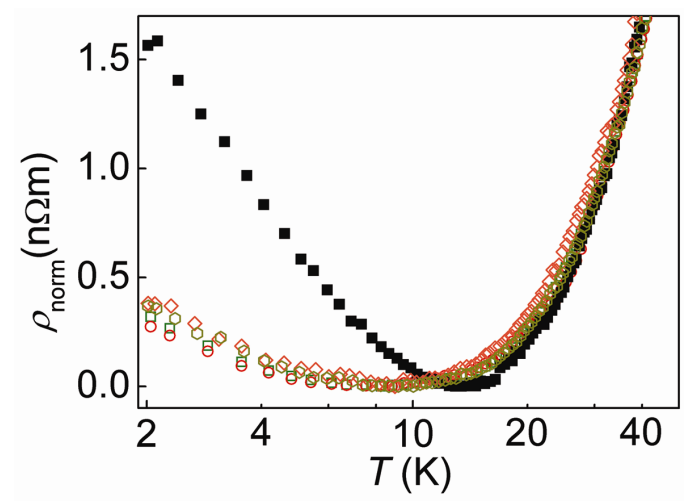

Figure 8.5: Temperature-dependent normalized resistivity plots of (99.99\%) pure Au films (green hexagons), and Au films with different ratios of $\mathrm{Co}$ and $\mathrm{Zn}$ complexes: $0 \%$ Co with top $\mathrm{Au}$ (orange diamonds) and without top Au (red circles), and $100 \%$ Co with top $\mathrm{Au}$ (black squares) and without top Au (green open squares).

\section{Sheet resistance of all samples for magnetoresistance measurement:}

The sheet resistance of all samples at $150 \mathrm{~K}$ for magnetoresistance measurement is given in Table 8.1. The standard deviation in the sheet resistance of all samples is $29.5 \Omega / \square$. The variation in sheet resistance may imply slight differences in the morphology of the gold films, which may contribute to both the inelastic and elastic scattering of the conduction electrons $[4,26]$. However, within this standard deviation, the shape of the magnetoresistance curve is dominated by magnetic scattering introduced via the Co complexes.

Table 8.1: Sheet resistance at $150 \mathrm{~K}$ of all samples for magnetoresistance measurement.

\begin{tabular}{|l|c|c|c|c|c|}
\hline Co-complex concentration (\%) & 0 & 25 & 50 & 67 & 100 \\
\hline Sheet resistance at $150 \mathrm{~K}(\Omega / \square)$ & 106.9 & 152.3 & 172.8 & 173.0 & 177.9 \\
\hline
\end{tabular}

\section{References}

[1] J. Kondo, Progress of Theoretical Physics 32 (1964) 37.

[2] M. A. Ruderman, C. Kittel, Physical Review 96 (1954) 99.

[3] T. Dietl, H. Ohno, Materials Today 9 (2006) 18. 
[4] G. Bergmann, in 50 Years of Anderson Localization, ed E. Abrahams, World Scientific (2010).

[5] J. W. Loram, T. E. Whall, P. J. Ford, Physical Review B 2 (1970) 857.

[6] B. de Boer, A. Hadipour, M. M. Mandoc, T. van Woudenbergh, P. W. M. Blom, Advanced Materials 17 (2005) 621.

[7] W. Chen, D. Qi, X. Gao, A. T. S. Wee, Progress in Surface Science 84 (2009) 279.

[8] E. C. P. Smits, S. G. J. Mathijssen, P. A. van Hal, S. Setayesh, T. C. T. Geuns, K. A. H. A. Mutsaers, E. Cantatore, H. J. Wondergem, O. Werzer, R. Resel, M. Kemerink, S. Kirchmeyer, A. M. Muzafarov, S. A. Ponomarenko, B. de Boer, P. W. M. Blom, D. M. de Leeuw, Nature 455 (2008) 956.

[9] B. Lee, Y. Chen, F. Duerr, D. Mastrogiovanni, E. Garfunkel, E. Y. Andrei, V. Podzorov, Nano Letters 10 (2010) 2427.

[10] J. C. Ho, R. Yerushalmi, Z. A. Jacobson, Z. Fan, R. L. Alley, A. Javey, Nature Materials 7 (2008) 62.

[11] W. P. Voorthuijzen, M. D. Yilmaz, W. J. M. Naber, J. Huskens, W. G. van der Wiel, Advanced Materials 23 (2011) 1346.

[12] The results of one series of samples are presented. These samples were prepared in the same Au deposition run, but the data are to very high degree reproducible for more than 5 independent fabrication runs.

[13] S. Kremer, W. Henke, D. Reinen, Inorganic Chemistry 21 (1982) 3013.

[14] J. Park, A. N. Pasupathy, J. I. Goldsmith, C. Chang, Y. Yaish, J. R. Petta, M. Rinkoski, J. P. Sethna, H. D. Abruna, P. L. McEuen, D. C. Ralph, Nature 417 (2002) 722.

[15] It was found that the deposition of the top layer is essential for getting sufficient interaction between the spin dopants and the conduction electrons in Au. The organic ligands were expected to be (partly) destroyed in this process.

[16] V. Madhavan, W. Chen, T. Jamneala, M. F. Crommie, N. S. Wingreen, Science 280 (1998) 567. 
[17]J. T. Li, W. D. Schneider, R. Berndt, B. Delley, Physical Review Letters 80 (1998) 2893.

[18] D. Goldhaber-Gordon, H. Shtrikman, D. Mahalu, D. Abusch-Magder, U. Meirav, M. A. Kastner, Nature 391 (1998) 156.

[19] W. G. van der Wiel, S. De Franceschi, T. Fujisawa, J. M. Elzerman, S. Tarucha, L. P. Kouwenhoven, Science 289 (2000) 2105.

[20] E. L. Altshuler, B. L. Altshuler, A. G. Aronov, Solid State Communications 54 (1985) 617.

[21] G. Chen, N. Giordano, Physical Review Letters 66 (1991) 209.

[22] D. R. Hamann, Physical Review 158 (1967) 570.

[23] P. L. Taylor, O. Heinonen, A Quantum Approach to Condensed Matter Physics Cambridge University Press (2002)

[24] A. Mordijck, I. Deckers, M. Labro, Journal of Magnetism and Magnetic Materials 104 (1992) 2081.

[25] W. Wei, R. Rosenbaum, G. Bergmann, Physical Review B 39 (1989) 4568.

[26] G. Dumpich, A. Carl, Physical Review B 43 (1991) 12074.

[27] S. Hikami, A. I. Larkin, Y. Nagaoka, Progress of Theoretical Physics 63 (1980) 707.

[28] M. Maskus, H. D. Abruna, Langmuir 12 (1996) 4455.

[29] V. Chechik, H. J. Wellsted, A. Korte, B. C. Gilbert, H. Caldararu, P. Ionita, A. Caragheorgheopol, Faraday Discussions 125 (2004) 279.

[30] D. V. J. Gosser, Cyclic Voltammetry: Simulation and analysis of reaction mechanisms VCH (1993) 
Page | 120 


\section{Appendix}

\section{Appendix A}

Design map of the e-beam structure for $A B$ rings.

\begin{tabular}{|c|c|c|c|c|c|c|c|}
\hline & $1-4$ & $5-8$ & $9-12$ & $13-16$ & $17-20$ & $21-24$ & Dimension (nm) \\
\hline A & 0 & 0 & 1 & 1 & 2 & 2 & space size \\
\hline B & 300 & 300 & 300 & 300 & 300 & 300 & width \\
\hline C & 3 & 3 & 4 & 4 & 5 & 5 & space size \\
\hline D & 400 & 400 & 400 & 400 & 400 & 400 & width \\
\hline E & 6 & 6 & 7 & 7 & 8 & 8 & space size \\
\hline F & 500 & 500 & 500 & 500 & 500 & 500 & width \\
\hline G & 9 & 9 & 10 & 10 & 12 & 12 & space size \\
\hline H & 600 & 600 & 600 & 600 & 600 & 600 & width \\
\hline I & 0 & 2 & 4 & 6 & 8 & 10 & space size \\
\hline J & 700 & 700 & 700 & 700 & 700 & 700 & width \\
\hline K & 80 & 80 & 70 & 70 & 60 & 60 & gap size \\
\hline L & 800 & 800 & 800 & 800 & 800 & 800 & width \\
\hline M & 50 & 50 & 40 & 40 & 30 & 30 & gap size \\
\hline N & 900 & 900 & 900 & 900 & 900 & 900 & width \\
\hline O & 20 & 20 & 10 & 10 & 0 & 0 & gap size \\
\hline P & 1000 & 1000 & 1000 & 1000 & 1000 & 1000 & width \\
\hline Q & 0 & 0 & 20 & 40 & 60 & 80 & gap size \\
\hline
\end{tabular}




\section{Appendix B}

Mask layout for contact pads

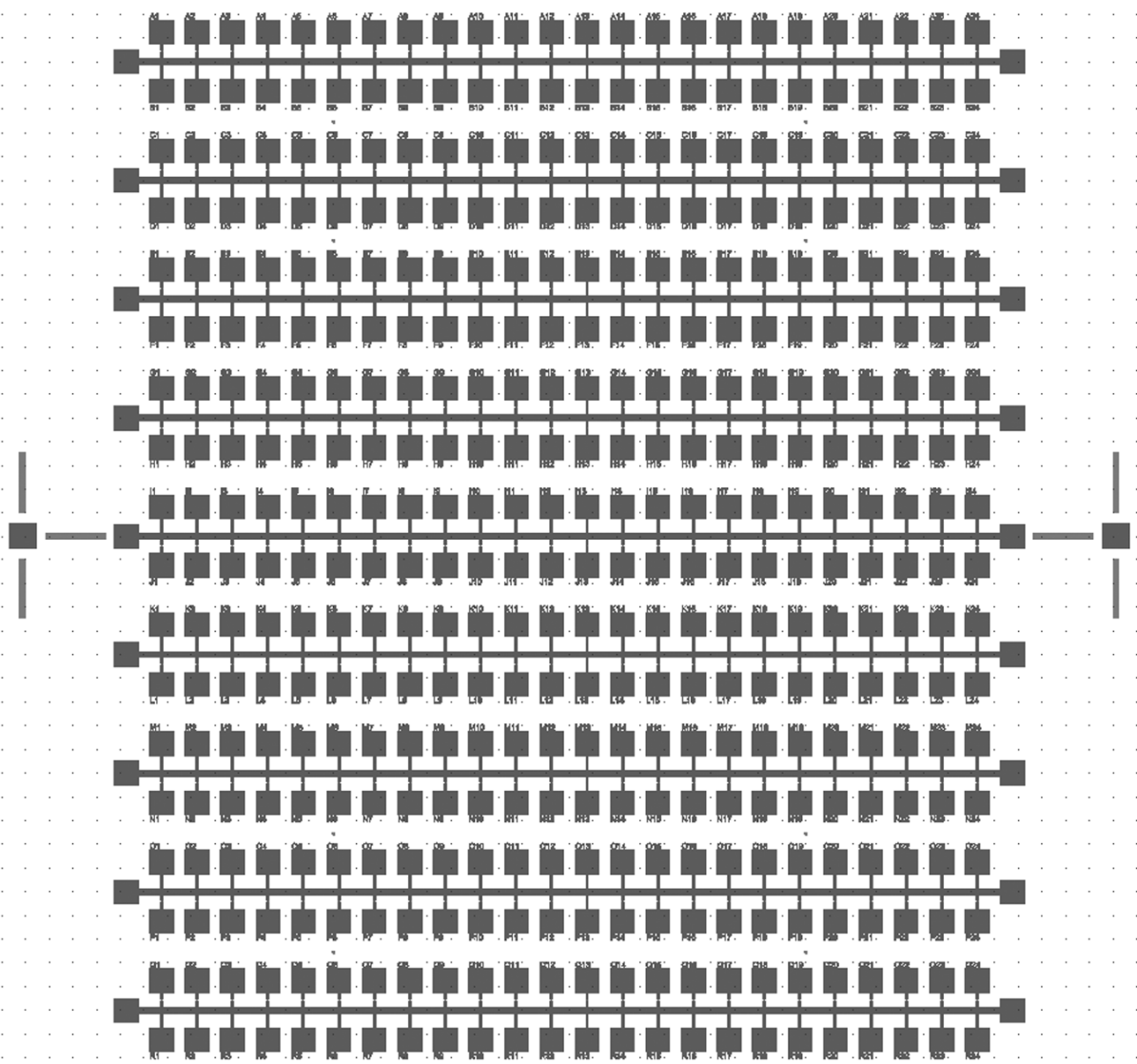




\section{Appendix C}

Calculation of aspect ratio and $A B$ period of a ring

As unsymmetrical ring is used for this project, it is not possible to give any general formula for the aspect ratio or the $A B$ period. The aspect ratio and the $A B$ period of the rings are calculated from the design file. We divided our hexagon as triangle and rectangle.

Effective area $\left(S_{\mathrm{in}}\right)=2 \times$ area of the triangle + area of the rectangle, Area of the arm $\left(S_{\text {arm }}\right)=$ circumference $\times$ width of the arm, Aspect ratio $=S_{\text {in }} / S_{\text {arm }}$

The expected $\mathrm{AB}$ period $\Delta B=\frac{h}{e S_{\text {in }}}$, where $h$ is Planck's constant, $e$ is elementary charge and $S_{\text {in }}$ is the effective area of the hexagon ring.

For instance, the design values from a $600 \mathrm{~nm}$ width ring are given by the following design file.

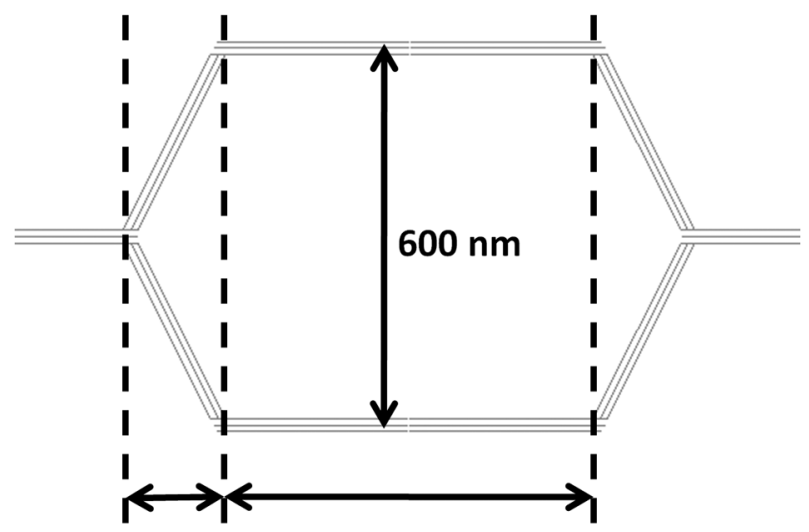

$163 \mathrm{~nm}$

$587 \mathrm{~nm}$

Area of the triangle $=\frac{1}{2} \times 600 \mathrm{~nm} \times 163 \mathrm{~nm}=4.89 \times 10^{-14} \mathrm{~m}^{2}$, Area of the rectangle $=600 \mathrm{~nm} \times 587 \mathrm{~nm}=3.522 \times 10^{-13} \mathrm{~m}^{2}$, $S_{\text {in }}=\left(2 \times 4.894 .89 \times 10^{-14}\right)+3.522 \times 10^{-13}=4.5 \times 10^{-13} \mathrm{~m}^{2}$, then the $\mathrm{AB}$ period is $\Delta B=\frac{h}{e S_{\mathrm{in}}}=\frac{6.626068 \times 10^{-34}}{1.602 \times 10^{-19} \times 4.5 \times 10^{-13}}=9.2 \mathrm{mT}$. 
The expected $A B$ periods for all the other rings are calculated in the same way. Aspect ratio and theoretical $A B$ oscillation period of the rings are listed below.

\begin{tabular}{|l|l|l|l|}
\hline Row & $\begin{array}{l}\text { Width of the } \\
\text { ring }(\mathrm{nm})\end{array}$ & $\begin{array}{l}\text { Aspect } \\
\text { ratio }\end{array}$ & $\begin{array}{l}\text { AB Period (mT) } \\
\text { theoretical }\end{array}$ \\
\hline B & 300 & 1.75 & 37.0 \\
\hline D & 400 & 2.38 & 20.7 \\
\hline F & 500 & 3.00 & 13.0 \\
\hline H & 600 & 3.63 & 9.2 \\
\hline J & 700 & 4.25 & 6.5 \\
\hline L & 800 & 4.88 & 5.0 \\
\hline N & 900 & 5.50 & 4.0 \\
\hline P & 1000 & 6.13 & 3.0 \\
\hline R & 1000 & 6.13 & 3.0 \\
\hline
\end{tabular}


Organic building blocks are increasingly applied in current nanoelectronics research and development. In this thesis, the investigation on several organic-inorganic hybrid electronic systems is presented. Several molecular approaches were developed to assemble magnetic nanoparticles on various substrates with controllable density. This is considered as a step forward towards nanoparticle based hybrid spintronics devices. An electron interferometer was developed and measured with the final purpose of studying electron coherent transport through molecular assemblies. At the end, a novel, facile molecular fabrication method for inserting isolated localized magnetic moments in a metal film with tunable density is presented. This offers a model system to study the mutual interaction of localized magnetic moments and their interplay with itinerant conduction electrons.

Chapter 2 provides a concise theoretical background for the magnetic and electrical phenomena in the organic-inorganic hybrid systems described in this thesis. These phenomena include nanomagnetism, spin-dependent transport, Coulomb blockade, the Kondo effect and electron interference.

Chapter 3 discusses the synthesis of uniform, isolated and patternable FePt(Au) nanoparticles that remain ferromagnetic at room temperature with potential in ultra-high density data storage applications. A systematic study of the preparation of the ferromagnetic nanoparticles in solution at relative low temperatures is carried out. The onset of the chemically ordered fct $\left(\mathrm{L1}_{0}\right)$ phase is obtained for thermal annealing at temperatures as low as $150{ }^{\circ} \mathrm{C}$. Large uniaxial magnetic anisotropy $\left(10^{7} \mathrm{erg} / \mathrm{cm}^{3}\right)$ and a high long-range order parameter have been obtained. This low-temperature solution annealing method preserves the organic ligands around the nanoparticles. This enables further chemically assisted assembly and patterning of these magnetic nanoparticles.

The assembly of a monolayer of magnetic nanoparticles on a surface using "click chemistry" is described in Chapter 4. Functionalized ligands on the nanoparticles and self-assembled monolayers (SAMs) modified on the substrates are used to 
link the magnetic nanoparticles on a silicon oxide substrate. The magnetic nanoparticles are assembled based on irreversible, covalent interaction. The nanoparticles are patternable via micro-contact printing $(\mu \mathrm{CP})$. This is promising in the light of using magnetic nanoparticles for spintronic and data storage applications.

Chapter 5 discusses well-controlled patterning and assembly methods to position the magnetic nanoparticles on the aluminum oxide or other metal oxide substrate. This is essential to realize nanoparticle based spintronic devices, since tunnel barriers consist of several nanometer of aluminum oxide or other metal oxide is widely used in numerous spintronic devices. By combining nanoimprint lithography or nano-molding in capillaries with SAMs of phosph(on)ate molecular linkers, FePtAu NP mono/multilayer patterns with high resolution below $150 \mathrm{~nm}$ as well as in micron range were demonstrated. High selectivity between the patterned and non-patterned regions was achieved.

Transport studies on single magnetic nanoparticles assembled on chemical modified metallic and oxide substrates are shown in Chapter 6. The deposition of isolated FePt magnetic nanoparticles on surfaces was achieved via the control of the rate and duration of the deposition process. Scanning tunneling microscopy in high vacuum and at low temperature was used to characterize a single magnetic nanoparticle electrically. At low temperature $(T \approx 40 \mathrm{~K})$, Coulomb blockade was observed. For a single nanoparticle, a Coulomb charging energy of $150 \mathrm{meV}$ was found, in agreement with the estimated particle capacitance. This study provides a promising measurement geometry to address isolated magnetic nanoparticles and study their (spin-dependent) transport properties.

In Chapter 7, an electron interferometer layout for coherent electron transport through organic molecules is discussed. An e-beam lithography defined sub-micrometer Aharonov-Bohm ( $A B$ ) ring was proposed as a probe for coherent electron transport. $A B$ oscillations were observed for rings with different sizes at cryogenic temperatures (down to $250 \mathrm{mK}$ ). The expected and measured $A B$ periods are in good agreement. A modified Aharonov-Bohm ring with a nano sized gap and a nano sized junction have been designed and fabricated. This may enable insertion of organic molecules into the electron interferometer via 
nanoparticle bridging. Then with an optimized balance junction, the coherency of transport through organic molecules could be investigated.

Finally, in Chapter 8, a two-dimensional spin system was fabricated using a molecular self assembly approach. The system consists of a disordered gold film with a monolayer of paramagnetic molecules embedded. The monolayer introduces a robust way to define the concentration of magnetic impurities in this two-dimensional spin system. Kondo and magnetoresistance measurements demonstrate that the impurity concentration in the metal is directly proportional to the molecular spin dopant concentration in solution. Although up to now it was widely believed that inter-impurity coupling sets in at $100 \mathrm{ppm}$ levels, this molecularly doped impurity systems show pure Kondo behaviour down to $2 \mathrm{~K}$ at impurity levels as high as $680 \mathrm{ppm}$. This technique can be a powerful way to reach unprecedented impurity concentrations, while avoiding undesired aggregation or segregation. This should pave the way for further study of spin phenomena that lie at the very heart of solid-state physics, such as the Kondo effect, RKKY interaction, spin glasses, and magnetic phase transitions.

To conclude, the assembly and magneto-electrical characterization of several hybrid organic-inorganic magnetically active systems were described. Several important issues still remain in the organic-inorganic hybrid systems. More control is required on the nature of organic-inorganic interface which is of crucial importance to transport properties. For instance, transport study in Chapter 6 demonstrates a good physical contact does not necessarily imply good electrical contact. Smarter assembly techniques with well controlled organic-inorganic interface still need to be further developed to fulfill its potential in organic-inorganic hybrid electronics. These challenges indicate ways to extend this thesis work in the future. 
Page | 128 


\section{Samenvatting}

Organische bouwstenen worden in toenemende mate toegepast in nano-elektronica onderzoek en ontwikkeling. In dit proefschrift wordt het onderzoek aan verscheidene organisch-anorganische hybride elektronische systemen gepresenteerd. Een aantal moleculaire methodes werd ontwikkeld om magnetische nanodeeltjes te assembleren op verschillende substraten met controleerbare dichtheid. Dit wordt beschouwd als een stap in de richting van hybride spintronische devices gebaseerd op nanodeeltjes. Een elektroninterferometer werd ontwikkeld en gekarakteriseerd met als uiteindelijk doel het bestuderen van coherent elektrontransport door moleculaire structuren. Ten slotte wordt er een nieuwe, eenvoudige moleculaire fabricagemethode gepresenteerd voor het doteren van een metaalfilm met geïsoleerde, gelocaliseerde magnetische momenten met regelbare dichtheid. Dit levert een modelsysteem op voor het bestuderen van de wederzijdse interactie van gelocaliseerde magnetische momenten en hun interactie met geleidingselektronen.

Hoofdstuk 2 verschaft een kort en bondige theoretische achtergrond voor de magnetische en elektronische fenomenen in de organisch-anorganische hybride systemen beschreven in dit proefschrift. Deze verschijnselen omvatten nanomagnetisme, spin-afhankelijk transport, Coulomb blokkade, het Kondo effect en elektroninterferentie.

Hoofdstuk 3 behandelt de synthese van uniforme, geïsoleerde, patroneerbare FePt(Au) nanodeeltjes die ferromagnetisch blijven bij kamertemperatuur met potentieel voor ultra-hoge-dichtheid dataopslagtoepassingen. Een systematische studie van de bereiding van deze ferromagnetische nanodeeltjes in oplossing bij relatief lage temperaturen is uitgevoerd. De chemisch geordende fct $\left(\mathrm{L}_{0}\right)$ fase begint zich al te vormen bij een bereidingstemperatuur van $150{ }^{\circ} \mathrm{C}$. Grote uniaxiale magnetische anisotropie $\left(10^{7} \mathrm{erg} / \mathrm{cm}^{3}\right)$ en een hoge lange-afstand ordeparameter zijn verkregen. Deze lage-temperatuur synthesemethode behoudt de organische liganden rondom de nanodeeltjes. Dit maakt verdere 
chemisch geassisteerde assemblage en patronering mogelijk van deze magnetische nanodeeltjes.

De assemblage van een monolaag magnetische nanodeeltjes op een oppervlak met behulp van "click chemie" is beschreven in Hoofdstuk 4. Gefunctionaliseerde liganden op de nanodeeltjes en zelf-geassembleerde monolagen (SAMs) gemodificeerd op het substraat worden gebruikt om de magnetische nanodeeltjes op een siliciumoxide substraat te verankeren. De magnetische nanodeeltjes worden geassembleerd gebaseerd op irreversibele, covalente interactie. De nanodeeltjes zijn patroneerbaar via micro-contact printing $(\mu \mathrm{CP})$. Dit is veelbelovend in het licht van spintronische en dataopslagtoepassingen.

Hoofdstuk 5 behandelt gecontroleerde patronering en assemblagemethodes om magnetische nanodeeltjes te positioneren op aluminiumoxide of andere metaaloxides. Dit is essentieel om spintronische devices gebaseerd op nanodeeltjes te maken, aangezien tunnelbarrières bestaande uit enkele nanometers aluminiumoxide dikwijls gebruikt worden in spintronische devices. Door het combineren van nanoimprintlithografie of "nano-molding in capillaries" met zelf-geassembleerde monolagen van fosf(on)aat moleculaire linkers, werden FePtAu nanodeeltjes mono/multilaag-patronen met hoge resolutie onder de 150 $\mathrm{nm}$ alsmede op de micronschaal gedemonstreerd. Hoge selectiviteit tussen de gepatroneerde en niet-gepatroneerde gedeeltes werd gerealiseerd.

Transportmetingen aan enkele magnetische nanodeeltjes geassembleerd op chemisch gemodificeerde metallische en oxidische substraten worden behandeld in Hoofdstuk 6. De depositie van geïsoleerde FePt nanodeeltjes op oppervlakken werd gerealiseerd door het controleren van de snelheid en duur van het depositieproces. Rastertunnelmicroscopie (STM) in hoog vacuüm en bij lage temperatuur werd gebruikt om een enkel magnetisch nanodeeltje elektrisch te karakteriseren. Bij lage temperatuur $(T \approx 40 \mathrm{~K})$ werd Coulomb blokkade geobserveerd. Voor een enkel nanodeeltje werd een ladingsenergie van $150 \mathrm{meV}$ gevonden, in overeenstemming met de geschatte capaciteit van het deeltje. Deze studie verschaft een veelbelovende meetgeometrie voor het adresseren van geïsoleerde magnetische nanodeeltjes en het bestuderen van hun (spin-afhankelijke) transporteigenschappen. 
In Hoofdstuk 7 wordt een elektroninterferometer layout voor coherent elektrontransport door organische moleculen behandeld. Een sub-micron Aharonov-Bohm ( $A B$ ) ring gedefinieerd door elektronenbundel-lithografie wordt voorgesteld als een test voor coherent elektrontransport. $A B$ oscillaties werden geobserveerd voor ringen met verschillende afmetingen bij cryogene temperaturen (tot $250 \mathrm{mK}$ ). De verwachte en gemeten $A B$ periodes kwamen goed overeen. Een gemodificeerde $A B$ ring met een nano-opening en nanojunctie werden ontworpen en gefabriceerd. Deze geometrie kan gebruikt worden voor het inbrengen van organische moleculen in de elektroninterferometer via "nanodeeltje-overbrugging". Met een geoptimaliseerde balansjunctie in de andere arm zou dan de coherentie van het transport door organische moleculen onderzocht kunnen worden.

Ten slotte, wordt er in Hoofdstuk 8 een 2-dimensionaal spinsysteem besproken, gefabriceerd door middel van een moleculaire zelf-assemblagemethode. Het systeem bestaat uit een wanorderlijke film van goud met een monolaag van paramagnetische moleculen ingebed. De monolaag vormt een robuuste manier om de concentratie magnetische onzuiverheden te controleren in dit 2-dimensionale spinsysteem. Kondo- en magnetoweerstandsmetingen tonen aan dat de onzuiverheidsconcentratie in het metaal direct schaalt met de concentratie moleculaire spindonoren in oplossing. Ook al werd er tot op heden alom geloofd dat koppeling tussen onzuiverheden begint bij 100 ppm niveaus, dit moleculair gedoteerde systeem laat puur Kondo-gedrag zien tot $2 \mathrm{~K}$ zelfs bij onzuiverheidsconcentraties van $680 \mathrm{ppm}$. Deze techniek kan een krachtige methode zijn om ongekende onzuiverheidsconcentraties te halen, zonder ongewenste aggregatie of segregatie. Dit zou verdere studie mogelijk moeten maken van spinverschijnselen in het hart van de vastestof-fysica, zoals het Kondo effect, RKKY-interactie, spinglassystemen en magnetische faseovergangen.

In conclusie, de assemblage en magneto-elektrische karakterisatie van verscheidene hybride organisch-anorgansiche, magnetisch actieve systemen is beschreven. Er bestaat nog altijd een aantal belangrijke uitdagingen in organisch-anorganische hybride systemen. Meer controle is noodzakelijk van het organisch-anorganische grensvlak, dat cruciaal is voor transporteigenschappen. De transportstudie in Hoofdstuk 6, bijvoorbeeld, laat zien dat goed mechanisch 
contact niet noodzakelijkerwijs goed elektrisch contact betekent. Slimmere assemblagetechnieken met goed gecontroleerde grensvlakken tussen de organische en anorganische materialen moeten verder ontwikkeld worden om het potentieel van organisch-anorganische hybride elektronica ten volle te benutten. Zulke uitdagingen vormen een uitgangspunt voor het vervolg van dit onderzoek in de toekomst. 


\section{Acknowledgements}

The appearance of this section means we are approaching the end of this thesis. Here the science is no longer the topic. It is all about emotions during my 6 years stay at the University of Twente. Beginning with a Master of Science program in Nanotechnology and ending as a Doctor of Philosophy, I had the opportunity to meet a lot of wonderful people to whom I am sincerely grateful. Without their support and encouragement, it would not have been possible.

This research position was offered to me by my supervisor and promotor, Wilfred van der Wiel. Thank you very much for creating this highly multidisciplinary research package. It is my great honor to share your enthusiasm, attitude, inspiring ideas, broad knowledge and vision in scientific research. Apart from those, you also give me a good example in time balancing, team working, teaching and management. I enjoyed it very much during the past four years.

It was also a great pleasure to have discussions with my other promotor, Dave Blank. I am thankful that I could benefit from your huge experience as a promotor and as a scientist.

Jurriaan, you were my supervisor during the master thesis project at the Molecular nanoFabrication (MnF) Group. Together with my daily supervisor Xingyi Ling, you trained me well. Although I left $\mathrm{MnF}$ and joined the NanoElectronics Group for the PhD position, the multidisciplinary nature of my project still binds us together. Thank you for offering me the facilities in the chemical lab and all the insightful discussions. I would like to thank Sego Tarucha for offering the high-quality e-beam samples. Without your help the work in Chapter 7 of this thesis would not have been possible. Mr. Yoshida, thanks for carrying out the fabrication work. Harold, your excessive experience on STM gave us quite a boost on the experiment described in Chapter 6. We really appreciate your advices. I would also like to thank all other members of my doctoral graduation committee for making time to evaluate this work, especially to those travelling from abroad. 
Wouter, we had many weekly meeting together, since we were the first two PhD candidates of Wilfred. I learned a lot from you: about physics, paperwork in daily life and even pop music. I also enjoyed our chats during the experiments. I wish you all the best!

Sachin, Oktay and Hans (Boschker), it was a nice experience to collaborate with you three on the FePt nanoparticle-related work described in Chapters 3, 4 and 5 of this thesis. Thank you for all the efforts you made for these chapters. I wish you all a great career.

Chapter 8 would not be part of this thesis without the cooperation of Deniz, Derya, Saurabh, Elia, Michel (de Jong), Aldrik and Jurriaan. Special thanks to Deniz for synthesis of the molecular complexes. Derya and Saurabh, I really enjoyed our very close teamwork together. I wish you both to be very fruitful with your projects.

Maarten, Mostafa and Sina, it was my great pleasure to have master students like you guys. I learned much by being your daily supervisor. Maarten, thanks for the excellent STM measurements. Mostafa and Sina, thanks for your help in the low temperature measurements. Interestingly, all of you chose to pursue the PhD degree after your master projects, I wish you all good luck with your research.

Joost and Menno, I had a wonderful time working with you two during the Heliox measurements. Sander and Erik, thanks for your help during the operation of the Heliox at $\mathrm{T} 2$.

Raymond, you are the lord of low noise measurement electronics. Thanks for your delicate creations. They allow us to explore the world of low temperature physics without blowing up our samples. Martin, Thijs and Johnny (Sanderink), you offered me great technical support at the NanoElectronics Group. Special thanks to Martin for his expertise on MFM measurements. Carolien, you fixed many administrative issues for me which made my 4 years PhD life much smoother. Richard and Marcel thanks for the technical support at the chemical lab. Richard, MOLINARD at Provence was a great place to visit. Thanks for your advice. I would also like to thank Rico for the TEM measurements, Mark for the TEM/SEM measurements and Gerard for the XPS measurements. Hans (Mertens), 
Rene and Robert, I really appreciate your support in the cleanroom.

I am especially grateful to Filipp and Michel (Zoontjes) for accepting to be my paranimfen. I would also like to thank Wilfred again for translating my Summary into Samenvatting. Ivan, Peter (Eerkes), Filipp and Michel (Zoontjes), together we made our office the best ever in the group. Last but not least, I want to express my thanks to all other (former) members of NanoElectronics Group for the supports and accompany during coffee breaks and NEvents. Good luck to all of you: Floris, Johnny (Wong), Ksenia, Serkan, Lan Ahn, Ina, Rabindra, Janine, Kai, Wen, Shahina, Jelle, Koert, Bernardus, Quyen, Lucky, Peter (Tijssen), Tamalika, Yunjae, Saroj, Sandeep and Ram.

Before moving on to acknowledge my friends, I would like to take this opportunity to thank Martin Bennink, Rik Akse and Julius Vancso for all their guidance and support during my master studies. I also appreciate the financial support offered by the Shell Centenary Scholarship Fund.

I would like to thank all the friends I met during the past 6 year in the Netherlands and around the world, who helped me a lot and shared a lot of wonderful and important moments with me. My cousin, Tiannan, it is nice to have you around in Europe. Hao and Lanti, being friends and neighbors we do have so much fun together! Zheng and Xiao, thanks for all the excellent cuisine. For Songyue and Yifan, our trips to Greece were fantastic! For Jens and Eef, our cycling tour in Hoge Veluwe National Park was unforgettable. Shan and Yuguo, you bridged me to the University of Twente 6 years ago. Especially Yuguo, thank you very much for helping me settle down in my first two years stay in Enschede. Sinie, thank you for the nice Sunday afternoons and all the Dutch news paper reading at your place. You connected Xin and me to the Dutch society. Joni, it was a nice time to stay at your place in the sunny California. Thanks a lot for all the arrangements you made for Xin and me. Shuo, the wedding presentation you made for Xin and me was fantastic. Wei (Cheng) and Zhiyu, I enjoyed our tennis games very much. Jing, Qi and Yujie, Chien-Ching and Kaifan, Xiaofeng and Yuying, Lingling and Mingliang, Henny and Wim, Yanbo, Weihua, Yiping, Wei (Zhou) and Wei (Zhao), Chunlin and Huaqiong, Weiqing, Feng, Chao, Di and many others, we had a lot of enjoyable time together and thank you all for your 
friendship!

Finally and most importantly, I would like to thank all my family members. I express my deepest gratitude to my grandmother Guifan Li, my parents Dapeng Gang and Dongge Xue. The last sentence belongs to my beloved wife Xin Wan and son Kevin W.Z. Gang. Thank you for your endless love, support and encouragement. 


\section{Author biography}

Tian Gang was born on December $19^{\text {th }} 1983$ in Changchun, P.R. China. After graduating from High School Attached to Northeast Normal University, he entered the College of Chemistry, Jilin University in 2001. In 2005, he obtained his Bachelor of Science degree in Materials Chemistry with the award of Excellent Graduation Thesis. He also obtained a Bachelor of Science degree in Biotechnology from the minor track at the College of Life Science, Jilin University. He joined the Master Program in Nanotechnology at the University of Twente in 2005. He was selected and financed by the Shell Centenary Scholarship Fund during his master studies. In 2007, he obtained his Master of Science degree in Nanotechnology under the supervision of Prof. Jurriaan Huskens in the Molecular NanoFabrication Group, University of Twente. In October 2007, he joined the NanoElectronics Group as a doctoral candidate under the supervision of Prof. Wilfred van der Wiel, working on the assembly and magneto-electrical characterization of hybrid organic-inorganic systems. In 2009, he got the Chinese Government Award for Outstanding Self-Financed Students Abroad. During this research he was also awarded a poster prize, a valorization prize and an invited talk at international conference. His doctoral research is described in this thesis. 
Page | 138 


\section{List of publications}

\section{Peer reviewed publications:}

Tunable Molecular Spin Doping of a Metal

T. Gang, M. Deniz Yilmaz, D. Ataç, S.K. Bose, E. Strambini, A.H. Velders, M.P. de Jong, J. Huskens and W.G. van der Wiel (2011), submitted

Nano-patterned monolayer and multilayer structures of FePtAu nanoparticles on aluminum oxide prepared by nanoimprint lithography and nanomolding in capillaries

T. Gang*, O. Yildirim*, S. Kinge, X. Duan, D. N. Reinhoudt, D. H. A. Blank, G. Rijnders, W. G. van der Wiel, J. Huskens, Journal of Materials Chemistry (2011), article in press. (* First authors)

Assembly and patterning of ferromagnetic nanoparticles from solution: A novel low-temperature liquid-phase annealing approach

T. Gang, M. Groen, S. Kinge, W. J. M. Naber, H. Boschker, G. Rijnders, D. N. Reinhoudt, W. G. van der Wiel, Synthetic Metals 161 (2011) 586.

Magnetic Nanoparticle Assembly on Surfaces Using Click Chemistry

S. Kinge, T. Gang, W. J. M. Naber, W. G. van der Wiel, D. N. Reinhoudt, Langmuir 27 (2010) 570. 
Monolayer-directed Assembly and Magnetic Properties of FePt Nanoparticles on Patterned Aluminum Oxide

O. Yildirim, T. Gang, S. Kinge, D. N. Reinhoudt, D. H. Blank, W. G. van der Wiel, G. Rijnders, J. Huskens, International Journal of Molecular Sciences 11 (2010) 1162.

Low-Temperature Solution Synthesis of Chemically Functional Ferromagnetic FePtAu Nanoparticles

S. Kinge ${ }^{*}$, T. Gang ${ }^{*}$, W. J. M. Naber, H. Boschker, G. Rijnders, D. N. Reinhoudt, W. G. van der Wiel, Nano Letters 9 (2009) 3220. (* First authors)

Multivalent Binding of Small Guest Molecules and Proteins to Molecular Printboards inside Microchannels

M. J. W. Ludden ${ }^{*}$, X. Y. Ling ${ }^{*}$, T. Gang, W. P. Bula, H. J. G. E. Gardeniers, D. N. Reinhoudt, J. Huskens, Chemistry: a European Journal 14 (2008) 136. (* First authors)

Ordered silica microspheres unsymmetrically coated with Ag nanoparticles, and Ag-nanoparticle-doped polymer voids fabricated by microcontact printing and chemical reduction

Z. M. Chen, T. Gang, X. Yan, X. Li, J. H. Zhang, Y. F. Wang, X. Chen, Z. Q. Sun, K. Zhang, B. Zhao, B. Yang, Advanced Materials 18 (2006) 924.

A simple method of preparing Ag nanoparticles coated silica colloidal crystals and polymer-Ag nanoparticles composite macroporous films

Z. M. Chen, T. Gang, Y. F. Wang, X. Chen, C. Guan, J. H. Zhang, Z. Q. Sun, K. Zhang, B. Zhao, B. Yang, Colloids and Surfaces a-Physicochemical and Engineering Aspects 277 (2006) 37. 
Ag nanoparticles-coated silica-PMMA core-shell microspheres and hollow PMMA microspheres with Ag nanoparticles in the interior surfaces

Z. M. Chen, T. Gang, K. Zhang, J. H. Zhang, X. Chen, Z. Q. Sun, B. Yang, Colloids and Surfaces a-Physicochemical and Engineering Aspects 272 (2006) 151.

A simple and controlled method of preparing uniform Ag midnanoparticles on Tollens-soaked silica spheres

Z. M. Chen, X. Chen, L. L. Zheng, T. Gang, T. Y. Cui, K. Zhang, B. Yang, Journal of Colloid and Interface Science 285 (2005) 146.

\section{Conference contributions:}

26th International Conference on Low Temperature Physics, July, 2011, Beijing, China. Invited Speaker in Parallel Sessions

MESA+ Annual Meeting, 2011, Enschede, The Netherlands. Poster presentation

SPINOS III, August, 2010, Amsterdam, The Netherlands. Oral presentation

STW Annual Congress, 2009,Nieuwegein, The Netherlands. Poster presentation

MicroNanoConference, November, 2008, Wageningen, The Netherlands. Oral presentation

53rd Conference on Magnetism and Magnetic Materials, November, 2008, Austin Texas, USA. Oral presentation 
Page | 142 